Supporting Information

\title{
Regioselective Single-Electron Tsuji-Trost Reaction of Allylic Alcohols: A Photoredox/Nickel Dual Catalytic Approach
}

\author{
Zheng-Jun Wang, ${ }^{\text {a,b }}$ Shuai Zheng, ${ }^{a}$ Eugenie Romero, ${ }^{a}$ Jennifer K. Matsui, ${ }^{a}$ and Gary A. Molander*a \\ ${ }^{\text {a }}$ Roy and Diana Vagelos Laboratories, Department of Chemistry, University of Pennsylvania, 231 S. $34^{\text {th }}$ Street, Philadelphia, Pennsylvania 19104- \\ 6323, United States \\ ${ }^{\mathrm{b}}$ State Key Laboratory of Chemo/Biosensing and Chemometrics, College of Chemistry and Chemical Engineering, Hunan University, Changsha \\ 41000, China \\ *Author to whom correspondence should be addressed. Email: gmolandr@sas.upenn.edu
}

\section{Contents}

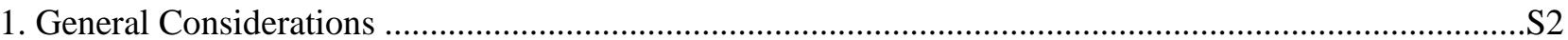

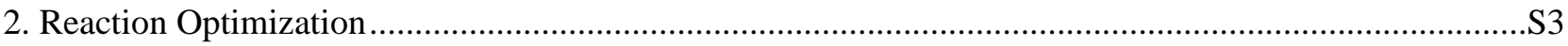

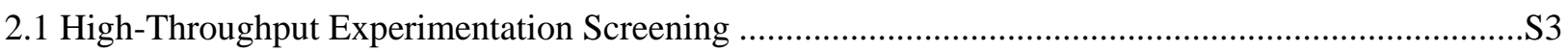

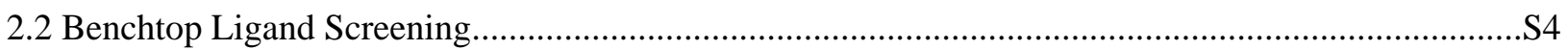

2.3 Substance Dependence on Alkylation of Allyl Alcohols ……...........................................................

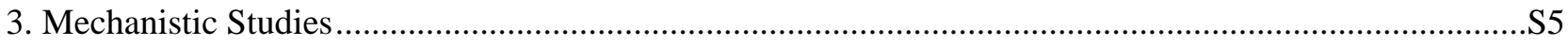

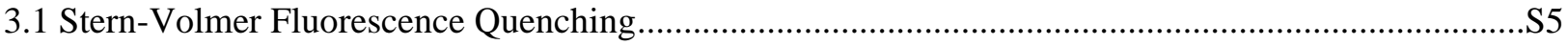

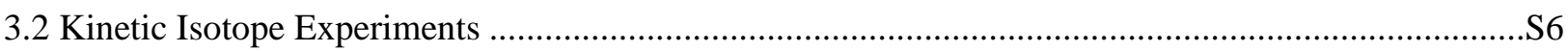

3.2.1 Synthesis and Characterization of Deuterated Substrate ..........................................................S6

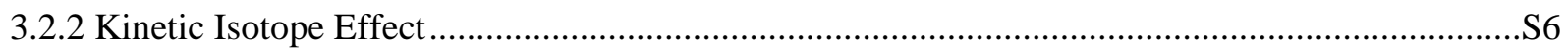

4. Photoredox/Nickel Dual-Catalyzed Alkylation of Allyl Alcohols and Carbonates ...............................S7

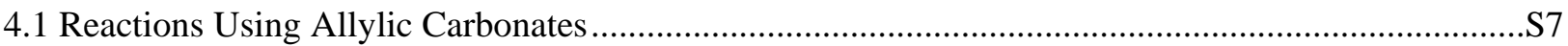

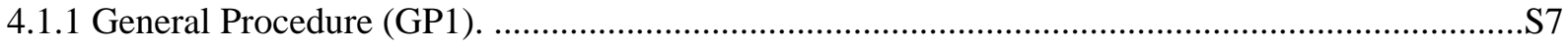

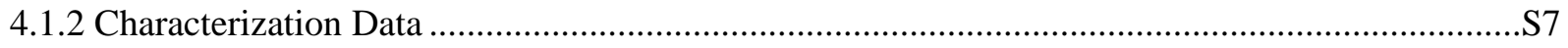

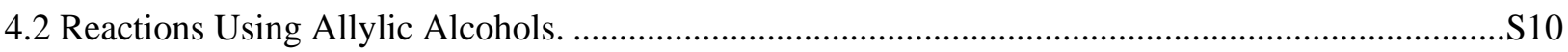

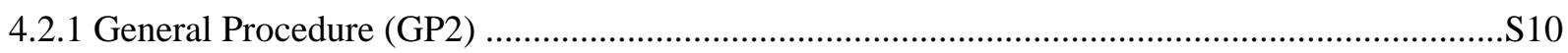

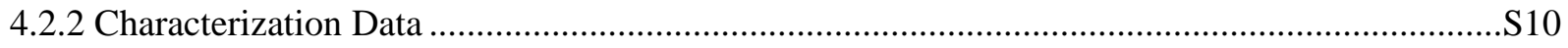

5. Photoredox/Nickel Dual-Catalyzed Sulfonylation of Allyl Carbonates.................................................S15

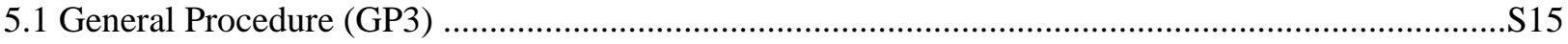

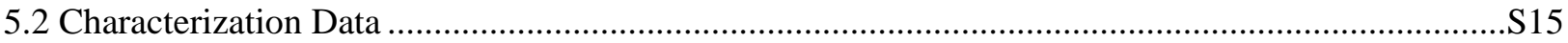

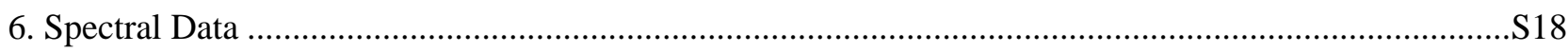

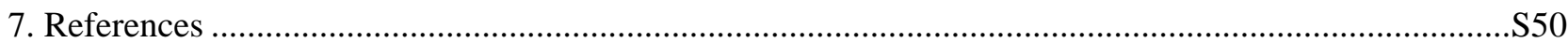




\section{General Considerations}

Unless otherwise noted, all reactions were carried out under an inert atmosphere of argon or nitrogen via standard Schlenk techniques or in a glovebox. Reactions were monitored by GC-MS, ${ }^{1} \mathrm{H}$ NMR, and/or by TLC on silica gel plates $\left(\mathrm{F}_{254}, 60 \AA\right)$. Thin layer chromatography was performed using hexanes/EtOAc as the eluents and visualized using $\mathrm{KMnO}_{4}$ or $p$-anisaldehyde stain and/or UV light. Reactions were purified by flash chromatography accompanied with an automated system (visualized at $254 \mathrm{~nm}$, monitored with allwavelength and ELS detector) with silica cartridges (60 A porosity, 20-40 $\mu \mathrm{m})$. Unless otherwise mentioned, all allylic alcohols were purchased from commercial sources and used as received. $\left[\operatorname{Ir}\left\{\mathrm{dF}\left(\mathrm{CF}_{3}\right)_{2} \mathrm{ppy}\right\}_{2}(\mathrm{bpy})\right]\left[\mathrm{PF}_{6}\right]^{1}$ and $4 \mathrm{CzIPN}^{2}$ were synthesized according to literature procedures. DMF, $\mathrm{MeCN}$ and acetone (extra dry, 99.8\%) were purchased and used as received. Alkyl 1,4-dihydropyridines ${ }^{3}$ were synthesized according to the literature. Allylic alcohols and carbonates were either purchased or synthesized according to literature procedures ${ }^{4}$ and matched with reported data. Other solvents were purified either by distillation over $\mathrm{Na}$ or $\mathrm{CaH}_{2}$ or by passing through alumina cartridges in a solvent purification system. Irradiation of reaction vessels was accomplished using a $5 \mathrm{~W}$ blue $(455 \mathrm{~nm})$ LED (light emitting diodes) strip about $3 \mathrm{~cm}$ from the reaction vessel with a fan above to maintain room temperature. The photoredox reaction equipment was constructed according to a previous report. ${ }^{5}$ Reaction optimization was carried out via high throughput experimentation and verified on the benchtop. Factors affecting reaction performance, such as solvents, photoredox catalysts, additives (e.g., transition metal, ligand, etc.), substrate loadings, as well as temperature have been thoroughly examined. NMR Spectra $\left({ }^{1} \mathrm{H},{ }^{13} \mathrm{C},{ }^{19} \mathrm{~F}\right)$ were recorded on a $500 \mathrm{MHz}$ spectrometer at $298 \mathrm{~K}$. All ${ }^{1} \mathrm{H}$ NMR spectra are reported in parts per million (ppm) downfield of TMS and were measured relative to the signal for $\mathrm{CHCl}_{3}(7.26 \mathrm{ppm})$. All ${ }^{13} \mathrm{C}$ NMR spectra are reported in ppm relative to residual $\mathrm{CHCl}_{3}(77.2 \mathrm{ppm})$ and were obtained with ${ }^{1} \mathrm{H}$ decoupling. All ${ }^{19} \mathrm{~F}$ NMR spectra were obtained in $\mathrm{CDCl}_{3}$ solution and are reported unreferenced. Coupling constants $(J)$ are reported in Hertz $(\mathrm{Hz})$. HRMS was obtained by either ESI or EI with a TOF spectrometer in $\mathrm{CH}_{3} \mathrm{CN}$ or $\mathrm{CHCl}_{3}$. The data were calibrated and reported by neutral atom masses, and the mass of electron is not included. IR spectra were obtained with neat samples. 


\section{Reaction Optimization}

\subsection{High-Throughput Experimentation Screening}

To a 96-well block with hollow bottom were added corresponding nickel sources (5 mol \%) and ligand (5 mol \%) for precomplexing. After removing the solvents, $p$-methoxy cinnamyl alcohol (1.0 equiv), dimethyl dicarbonate (DMDC, 3.0 equiv), solvents $(0.1 \mathrm{M})$, isopropyl dihydropyridine ( $i$-Pr DHP, 1.5 equiv), and photoredox catalysts $(3 \mathrm{~mol} \%$ ) were added to set up 96 parallel reactions on a $10 \mu \mathrm{mol}$ scale. The block was then put on a photoreactor for $16 \mathrm{~h}$. After completion, the mixture was diluted with $500 \mu \mathrm{L}$ of $\mathrm{MeCN}$ containing $0.002 \mathrm{M} 4,4$ '-di-tert-butyl-1,1'-biphenyl as an internal standard, and centrifuged, and $25 \mu \mathrm{L}$ of the clear solution was further diluted with $700 \mu \mathrm{L} \mathrm{MeCN}$ before being analyzed by UPLC. The product/internal standard ratio of the screening is shown below:

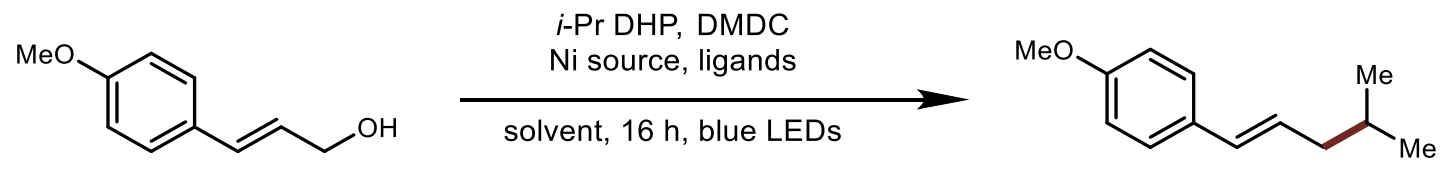

Whole Plate View - Product/IS

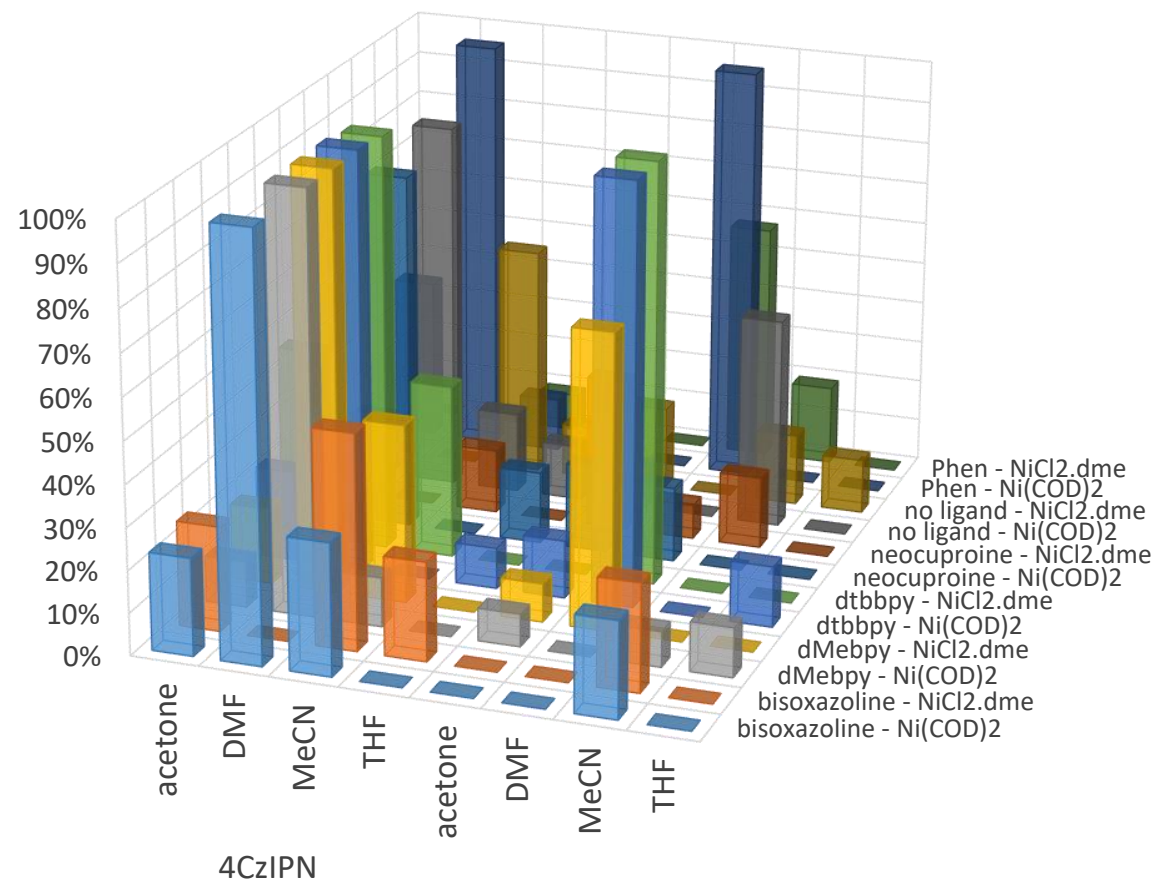

$\operatorname{Ir}[\mathrm{dFCF3ppy]2(bpy)PF6}$

Table S1 High Throughput Screening Results

From the screening result it is evident that DMF is the best solvent, and 4CzIPN rendered comparable yields to that of the more expensive Ir photocatalyst. Both $\mathrm{Ni}(\mathrm{COD})_{2}$ and $\mathrm{Ni}(\mathrm{dme}) \mathrm{Cl}_{2}$ gave good conversion, thus the air-stable $\mathrm{Ni}(\mathrm{II})$ salt is the more convenient choice. Several ligands led to good conversion into product, yet the $E / Z$ selectivity still needs to be validated on the benchtop. 


\subsection{Benchtop Ligand Screening}

$$
\text { Cy-DHP }
$$

$\begin{gathered}\text { DMF, blue LEDs } \\ 6 \mathrm{~h}, \mathrm{rt} \\ \text { deviation from standard conditions }\end{gathered}$
result $(\%$ yield $)$

Table S2 Benchtop Ligand Optimization

Ligands of different classes have been extensively investigated. Although the increase of steric bulk should favor the $E$-product formation, it is interesting that when using box-type ligands, the $Z$-product became predominant. Unfortunately, the overall yield could not be raised. Additionally, ligand-free conditions and phosphine ligands were also tested, neither of which gave good results.

\subsection{Substance Dependence on Alkylation of Allyl Alcohols}

During the substrate scope investigation of DMDC activation of allyl alcohols, several substrates exhibited diminished yield because of the formation of branched products and significant amounts of protodehydroxylation byproducts. Although the reason remains unclear, some examples still provided high regioselectivity using the prefunctionalized allyl carbonates as electrophiles (e.g., example 2c). Alkylsubstituted allyl carbonates and alcohols generally suffered from diminished reactivity. A table of challenging substrates is presented below:

The following allyl alcohols led to inseparable mixtures of protodehydroxylation/linear/branched products:<smiles>Cc1cc(C)c(/C=C/CO)c(C)c1</smiles><smiles>OC/C=C/c1cnc2ccccc2c1</smiles><smiles>OC/C=C/c1ccc2ccccc2c1</smiles><smiles>CC(O)C=Cc1ccccc1</smiles>

Alkyl-substituted allyl alcohols/carbonates suffered from low to no reactivity:

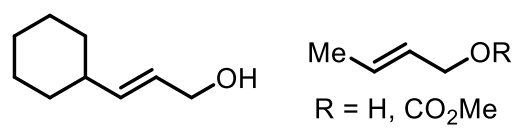

Persistent radicals rendered inseparable 1:1 linear/branched mixture:<smiles>CC(P)Cc1ccc2c(c1)OCO2</smiles><smiles>[PH]Cc1ccccc1</smiles><smiles>COC(OC)PP</smiles>

$3^{\circ}$ alkyl radical is unreactive:<smiles>CC(C)(C)P</smiles>

Table S3 Challenging Substrates 


\section{Mechanistic Studies}

\subsection{Stern-Volmer Fluorescence Quenching}

Stern-Volmer experiments were conducted on a Horiba Fluorolog® Spectrofluorometer. Stock solutions of substrates, photocatalyst, and base were prepared with dry acetone. The solutions were mixed and purged with argon for $30 \mathrm{sec}$ immediately prior to measurement. The samples were excited at $435 \mathrm{~nm}$, and emission data were recorded at $531 \mathrm{~nm}$. $\mathrm{I}_{0} / \mathrm{I}$ value of each sample were calculated from the average of three scans per data point.

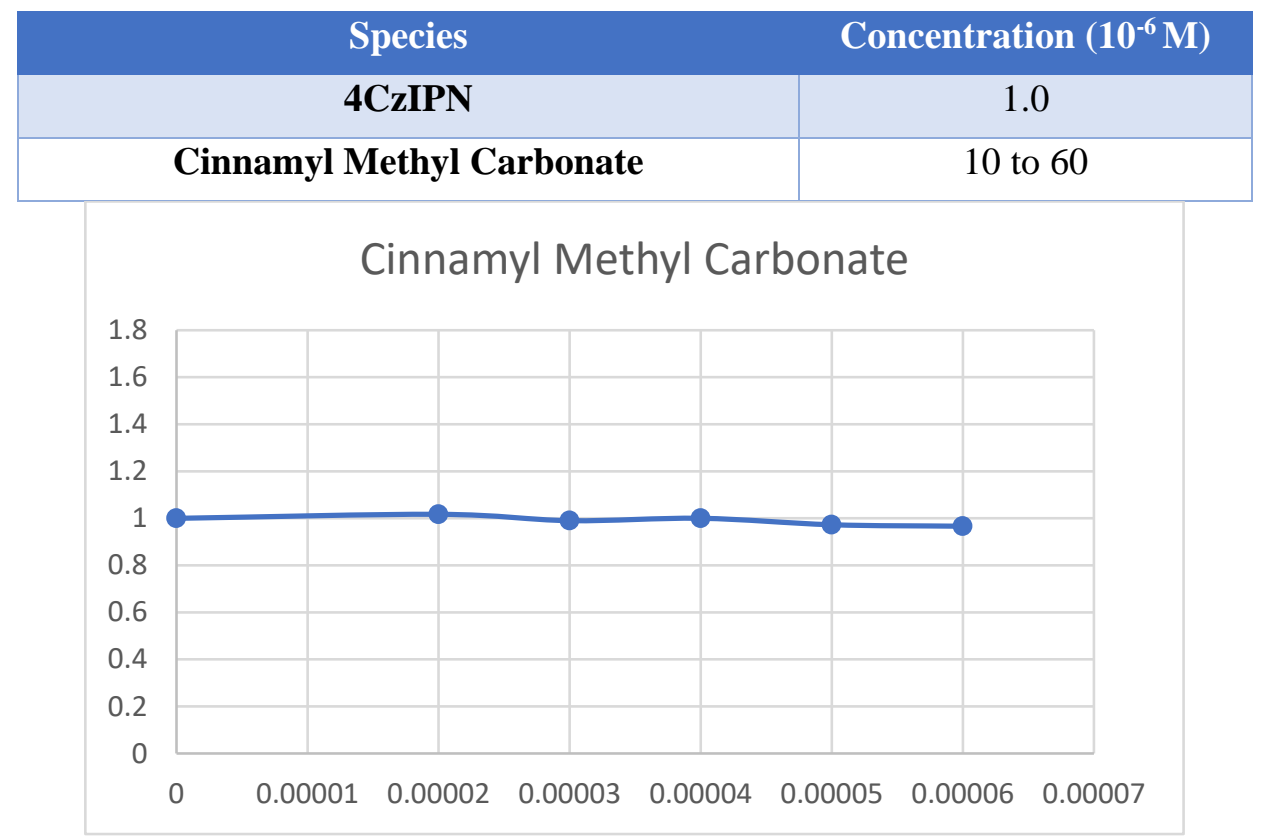

Figure S1 Fluorescence Quenching Studies with Allyl Carbonate

As indicated in the graph above, no significant quenching is observed with cinnamyl methyl carbonate, therefore it is unlikely that a radical-radical coupling pathway is operational in this reaction.

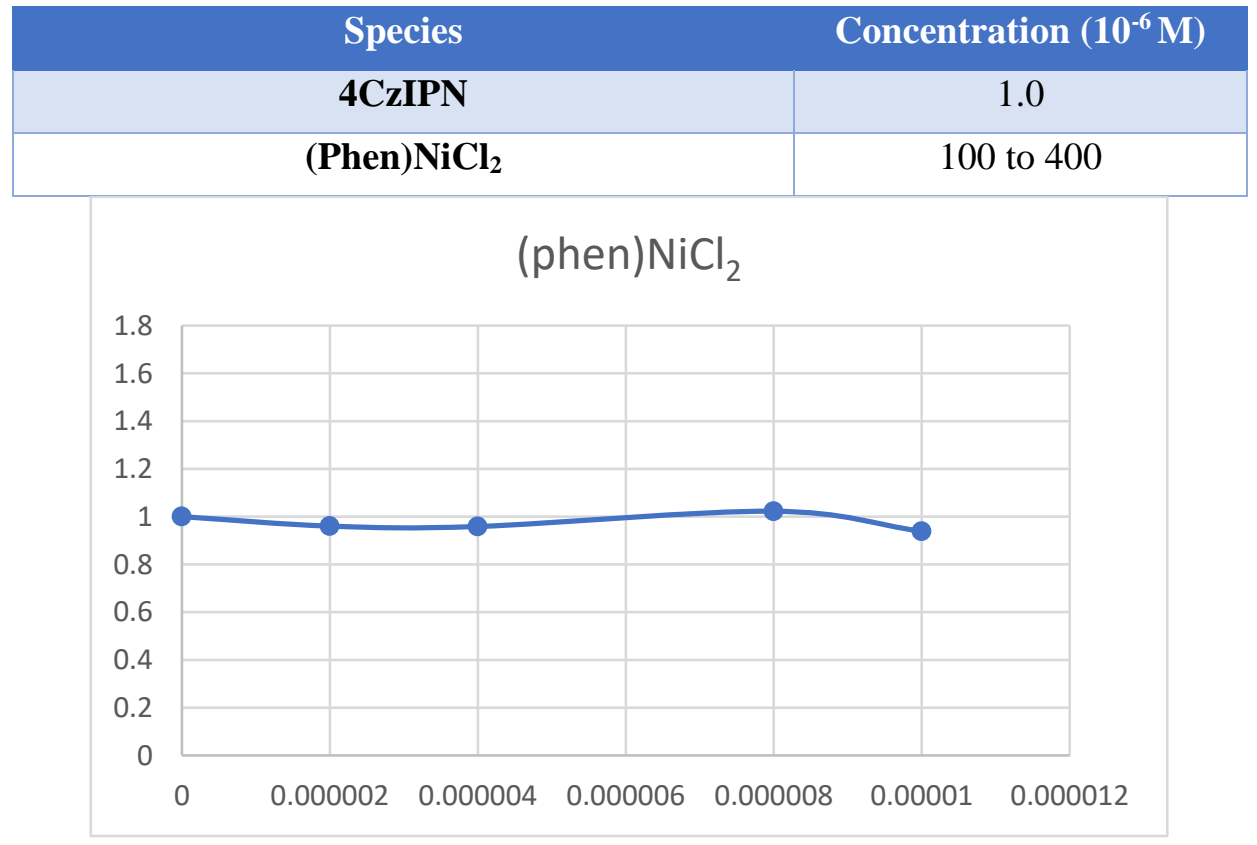


Figure S2 Fluorescence Quenching Studies with (phen) $\mathrm{NiCl}_{2}$

A recent report showed that Ir photocatalyst can be quenched by (bpy) $\mathrm{NiCl}_{2},{ }^{6}$ which indicated alternative pathways of activation of $\mathrm{Ni}$ precatalyst and oxidative addition of allyl carbonates. To test this possibility, we performed SternVolmer studies of (phen) $\mathrm{NiCl}_{2}$ on $4 \mathrm{CzIPN}$, mimicking the reaction conditions, but no significant quenching was observed. Therefore, it is unlikely that the Ni precatalyst is directly activated by excited photocatalyst 4CzIPN.

\subsection{Kinetic Isotope Experiments}

\subsubsection{Synthesis and Characterization of Deuterated Substrate}

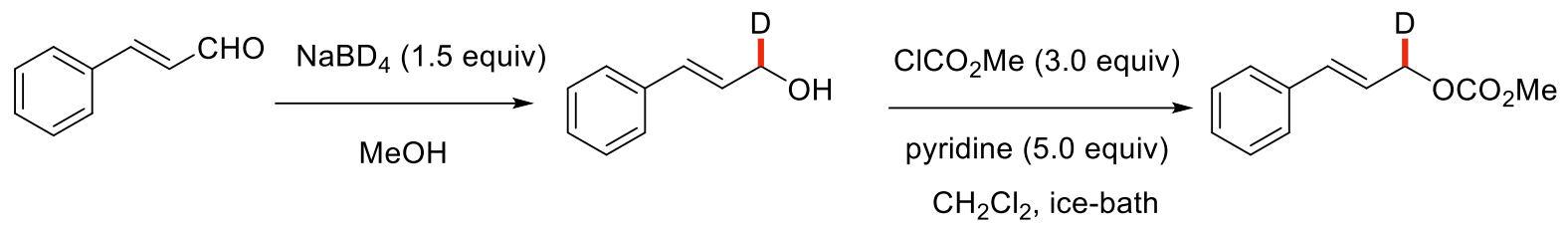

The $\alpha$-deuterated allyl methyl carbonate was synthesized via reducing cinnamyl aldehyde with $\mathrm{NaBD}_{4}$, following previous reports. ${ }^{4,7}$

(E)-Methyl (3-phenylallyl-1-d) carbonate (S1-d)<smiles>[2H]C(/C=C/c1ccccc1)OC(C)=O</smiles>

${ }^{1} \mathrm{H}$ NMR $\left(500 \mathrm{MHz}, \mathrm{CDCl}_{3}\right) \delta 7.44-7.38(\mathrm{~m}, 2 \mathrm{H}), 7.36-7.31(\mathrm{~m}, 2 \mathrm{H}), 7.30-7.26(\mathrm{~m}, 1 \mathrm{H}), 6.71(\mathrm{dd}, J=$ $15.8,1.3 \mathrm{~Hz}, 1 \mathrm{H}), 6.31(\mathrm{dd}, J=15.9,6.4 \mathrm{~Hz}, 1 \mathrm{H}), 4.83-4.74(\mathrm{~m}, 1 \mathrm{H}), 3.82(\mathrm{~s}, 3 \mathrm{H}) .{ }^{13} \mathrm{C}$ NMR $(126 \mathrm{MHz}$, $\left.\mathrm{CDCl}_{3}\right) \delta 155.8,136.2,135.0,128.7,128.3,126.8,122.5,68.2\left({ }^{2} J_{\mathrm{C}-\mathrm{D}}=22.4 \mathrm{~Hz}\right), 54.9$. IR (neat, $\left.\mathrm{cm}^{-1}\right): 3025$, 2950, 1754, 1497, 1441, 1346, 1250, 1130, 1068, 966, 924, 83, 791, 742, 691. HRMS (EI) calcd for $\mathrm{C}_{11} \mathrm{H}_{11} \mathrm{DO}_{3}[\mathrm{M}]^{+}$193.0849, found 193.0846.

\subsubsection{Kinetic Isotope Effect}

Kinetic isotopic effect experiments were carried out in the high throughput experimentation facility. Two 24well plates were used to set up 24 reactions in parallel, each with $\alpha$-deuterated and non-deuterated allyl carbonates. The reactions were stopped and worked up after $2 \mathrm{~h}$ and analyzed by UPLC using 10 mol \% 4,4'di-tert-butyl-1,1'-biphenyl as internal standard, and the data were analyzed using Excel.

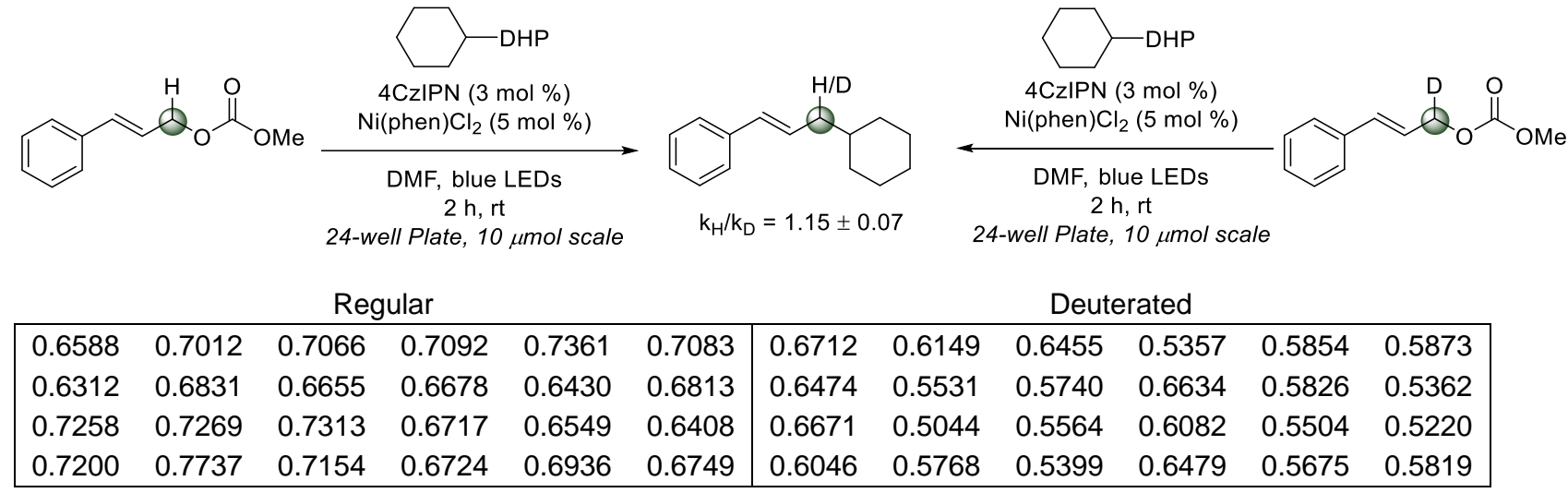

A secondary KIE is observed in this experiment, which indicated that oxidative addition might be the ratedetermining step. 


\section{Photoredox/Nickel Dual-Catalyzed Alkylation of Allyl Alcohols and Carbonates}

\subsection{Reactions Using Allylic Carbonates}

\subsubsection{General Procedure (GP1).}

To an 8 -mL reaction vial equipped with a stir bar and septa screw cap were added alkyl 1,4-dihydropyridines ( $0.45 \mathrm{mmol}, 1.5$ equiv), allylic carbonates ( $0.3 \mathrm{mmol}, 1.0$ equiv), (phen) $\mathrm{NiCl}_{2}(4.7 \mathrm{mg}, 5 \mathrm{~mol} \%$ ), and $4 \mathrm{CzIPN}$ (7.1 mg, $3 \mathrm{~mol} \%$ ). The reaction vial was subsequently closed, and three vacuum/argon cycles were performed, followed by addition of $3 \mathrm{~mL}$ of dry DMF $(0.1 \mathrm{M})$. After stirring under blue LEDs for $16 \mathrm{~h}$, the reaction was diluted with $30 \mathrm{~mL}$ of EtOAc and washed three times with $10 \mathrm{~mL}$ of saturated $\mathrm{Na}_{2} \mathrm{CO}_{3}$ and subsequently three times with $10 \mathrm{~mL}$ of brine. The organic phase was dried $\left(\mathrm{MgSO}_{4}\right)$, and the solvent was removed by rotovap. The final product was purified by flash column chromatography, using hexanes and EtOAc as eluent.

\subsubsection{Characterization Data}

(E)-(3-Cyclohexylprop-1-en-1-yl)benzene (2a \& 3a)<smiles>C(=Cc1ccccc1)CC1CCCCC1</smiles>

Following general procedure GP1 using cinnamyl methyl carbonate $(57.7 \mathrm{mg}, 0.3 \mathrm{mmol}, 1.0$ equiv), diethyl 4-cyclohexyl-2,6-dimethyl-1,4-dihydropyridine-3,5-dicarboxylate (150.9 $\mathrm{mg}, 0.45 \mathrm{mmol}, 1.5$ equiv), (phen) $\mathrm{NiCl}_{2}(4.7 \mathrm{mg}, 5 \mathrm{~mol} \%)$, and 4CzIPN (7.1 mg, $3 \mathrm{~mol} \%$ ) in DMF (3 mL, $\left.0.1 \mathrm{M}\right)$. The reaction was stirred under blue LEDs at $\mathrm{rt}$ for $16 \mathrm{~h}$. Silica gel purification using an automated system (hexanes/EtOAc, 0 to $10 \%)$ gave the product as a colorless oil $(52.3 \mathrm{mg}, 87 \%) .{ }^{1} \mathrm{H}$ NMR $\left(500 \mathrm{MHz}, \mathrm{CDCl}_{3}\right) \delta 7.35(\mathrm{~d}, J=7.6$ $\mathrm{Hz}, 2 \mathrm{H}), 7.29$ (dd, $J=7.6,7.4 \mathrm{~Hz}, 2 \mathrm{H}), 7.19(\mathrm{t}, J=7.4 \mathrm{~Hz}, 1 \mathrm{H}), 6.36(\mathrm{~d}, J=15.8 \mathrm{~Hz}, 1 \mathrm{H}), 6.23$ (dt, $J=15.3$, $7.2 \mathrm{~Hz}, 1 \mathrm{H}), 2.11(\mathrm{t}, J=7.1 \mathrm{~Hz}, 2 \mathrm{H}), 1.78-1.71(\mathrm{~m}, 4 \mathrm{H}), 1.66(\mathrm{~d}, J=12.5 \mathrm{~Hz}, 1 \mathrm{H}), 1.45-1.37(\mathrm{~m}, 1 \mathrm{H}), 1.29-$ $1.13(\mathrm{~m}, 3 \mathrm{H}), 1.01-0.93(\mathrm{~m}, 2 \mathrm{H}) .{ }^{13} \mathrm{C}$ NMR $\left(126 \mathrm{MHz}, \mathrm{CDCl}_{3}\right) \delta 138.1,130.8,129.9,128.6,126.9,126.0$, 41.2, 38.4, 33.4, 26.7, 26.5. IR (neat, $\mathrm{cm}^{-1}$ ): 3059, 3025, 2919, 2850, 1599, 1496, 1447, 1349, 1262, 1071, 1028, 963, 940, 909, 890, 846, 775, 739, 691, 513. HRMS (EI) calcd for $\mathrm{C}_{15} \mathrm{H}_{20}[\mathrm{M}]^{+} 200.1565$, found 200.1557. A 1-mmol scale reaction was carried out using cinnamyl methyl carbonate $(192.3 \mathrm{mg}, 1.0 \mathrm{mmol}$, 1.0 equiv), diethyl 4-cyclohexyl-2,6-dimethyl-1,4-dihydropyridine-3,5-dicarboxylate (503.1 mg, $1.5 \mathrm{mmol}$, 1.5 equiv), (phen) $\mathrm{NiCl}_{2}$ (15.5 mg, $5 \mathrm{~mol} \%$ ), and 4CzIPN (23.7 mg, $3 \mathrm{~mol} \%$ ) in DMF (10 mL, $\left.0.1 \mathrm{M}\right)$. The reaction was stirred under blue LEDs at $\mathrm{rt}$ for $16 \mathrm{~h}$. Silica gel purification using an automated system (hexanes/EtOAc, 0 to $10 \%$ ) gave the product as a colorless oil $(170.2 \mathrm{mg}, 85 \%) .{ }^{1} \mathrm{H}$ and ${ }^{13} \mathrm{C}$ NMR spectra match with the reported data above.

\section{(E)-(4-Methylpent-1-en-1-yl)benzene (2b \& 3b)}<smiles>CC(C)CC=Cc1ccccc1</smiles>

Following general procedure GP1 using cinnamyl methyl carbonate ( $57.7 \mathrm{mg}, 0.3 \mathrm{mmol}, 1.0$ equiv), diethyl 4-isopropyl-2,6-dimethyl-1,4-dihydropyridine-3,5-dicarboxylate (146.3 mg, $0.45 \mathrm{mmol}, 1.5$ equiv), (phen) $\mathrm{NiCl}_{2}(4.7 \mathrm{mg}, 5 \mathrm{~mol} \%)$, and 4CzIPN (7.1 mg, $\left.3 \mathrm{~mol} \%\right)$ in DMF (3 mL, $\left.0.1 \mathrm{M}\right)$. The reaction was stirred under blue LEDs at $\mathrm{rt}$ for $16 \mathrm{~h}$. Silica gel purification using an automated system (hexanes/EtOAc, 0 to $10 \%)$ gave the product as a colorless oil $(35.1 \mathrm{mg}, 73 \%) .{ }^{1} \mathrm{H} \mathrm{NMR}\left(500 \mathrm{MHz}, \mathrm{CDCl}_{3}\right) \delta 7.40-7.36(\mathrm{~m}$, $2 \mathrm{H}), 7.32(\mathrm{dd}, J=7.7,7.7 \mathrm{~Hz}, 2 \mathrm{H}), 7.24-7.19(\mathrm{~m}, 1 \mathrm{H}), 6.41(\mathrm{~d}, J=15.5 \mathrm{~Hz}, 1 \mathrm{H}), 6.25(\mathrm{dt}, J=15.6,7.2 \mathrm{~Hz}$, $1 \mathrm{H}), 2.13(\mathrm{ddd}, J=7.0,7.0,1.4 \mathrm{~Hz}, 2 \mathrm{H}), 1.80-1.74(\mathrm{~m}, 1 \mathrm{H}), 0.98(\mathrm{~d}, J=6.5 \mathrm{~Hz}, 6 \mathrm{H}) .{ }^{13} \mathrm{C} \mathrm{NMR}(126 \mathrm{MHz}$, $\left.\mathrm{CDCl}_{3}\right) \delta 138.1,131.0,130.0,128.6,126.9,126.1,42.6,28.8,22.5$. IR (neat, $\left.\mathrm{cm}^{-1}\right): 3028,2962,2933,2875$, 1749, 1712, 1496, 1406, 1368, 1267, 1124, 1071, 1019, 965, 908, 731, 693, 648, 498. HRMS (EI) calcd for $\mathrm{C}_{12} \mathrm{H}_{16}[\mathrm{M}]^{+}$160.1252, found 160.1247 .

(E)-2-(4-Methylpent-1-en-1-yl)naphthalene (2c) 
<smiles>CC(C)C/C=C/c1ccc2ccccc2c1</smiles>

Following general procedure GP1 using $(E)$-methyl (3-(naphthalen-2-yl)allyl) carbonate $(72.7 \mathrm{mg}, 0.3 \mathrm{mmol}$, 1.0 equiv), diethyl 4-isopropyl-2,6-dimethyl-1,4-dihydropyridine-3,5-dicarboxylate (146.3 $\mathrm{mg}, 0.45 \mathrm{mmol}$, 1.5 equiv), (phen) $\mathrm{NiCl}_{2}$ (4.7 mg, $5 \mathrm{~mol} \%$ ), and 4CzIPN (7.1 mg, $3 \mathrm{~mol} \%$ ) in DMF ( $\left.3 \mathrm{~mL}, 0.1 \mathrm{M}\right)$. The reaction was stirred under blue LEDs at $\mathrm{rt}$ for $16 \mathrm{~h}$. Silica gel purification using an automated system (hexanes/EtOAc, 0 to $10 \%)$ gave the product as a colorless oil $(46.7 \mathrm{mg}, 74 \%) .{ }^{1} \mathrm{H} \mathrm{NMR}\left(500 \mathrm{MHz}, \mathrm{CDCl}_{3}\right)$ $\delta$ 7.81-7.76 (m, 3H), $7.69(\mathrm{~s}, 1 \mathrm{H}), 7.60(\mathrm{dd}, J=8.5,1.5 \mathrm{~Hz}, 1 \mathrm{H}), 7.48-7.40(\mathrm{~m}, 2 \mathrm{H}), 6.55(\mathrm{~d}, J=15.5 \mathrm{~Hz}, 1 \mathrm{H})$, 6.40-6.33 (m, 1H), $2.17(\mathrm{dt}, J=7.2,1.0 \mathrm{~Hz}, 2 \mathrm{H}), 1.81-1.75(\mathrm{~m}, 1 \mathrm{H}), 0.99(\mathrm{~d}, J=6.5 \mathrm{~Hz}, 6 \mathrm{H}) .{ }^{13} \mathrm{C}$ NMR $(126$ $\left.\mathrm{MHz}, \mathrm{CDCl}_{3}\right) \delta 135.3,133.6,130.8,130.3,128.7,127.9,127.7,127.5,126.0,125.3,125.2,123.5,42.5,28.6$, 22.3. IR (neat, $\mathrm{cm}^{-1}$ ): 3056, 3019, 2953, 2925, 2868, 1598, 1508, 1464, 1435, 1383, 1366, 1271, 1167, 965 , 892, 857, 815, 785, 741, 475. HRMS (EI) calcd for $\mathrm{C}_{16} \mathrm{H}_{18}[\mathrm{M}]^{+} 210.1409$, found 210.1405.

(E)-1-Fluoro-4-(4-methylpent-1-en-1-yl)benzene (2d)<smiles>CC(C)C/C=C/c1ccc(F)cc1</smiles>

Following general procedure GP1 using (E)-3-(4-fluorophenyl)allyl methyl carbonate $(63.1 \mathrm{mg}, 0.3 \mathrm{mmol}$, 1.0 equiv), diethyl 4-isopropyl-2,6-dimethyl-1,4-dihydropyridine-3,5-dicarboxylate (146.3 $\mathrm{mg}, 0.45 \mathrm{mmol}$, 1.5 equiv), (phen) $\mathrm{NiCl}_{2}(4.7 \mathrm{mg}, 5 \mathrm{~mol} \%$ ), and 4 CzIPN (7.1 mg, $3 \mathrm{~mol} \%$ ) in DMF ( $3 \mathrm{~mL}, 0.1 \mathrm{M})$. The reaction was stirred under blue LEDs at $\mathrm{rt}$ for $16 \mathrm{~h}$. Silica gel purification using an automated system (hexanes/EtOAc, 0 to $10 \%$ ) gave the product as a colorless oil $(40.5 \mathrm{mg}, 76 \%) .{ }^{1} \mathrm{H} \mathrm{NMR} \mathrm{(500} \mathrm{MHz}, \mathrm{CDCl}_{3}$ ) $\delta 7.35-7.29(\mathrm{~m}, 2 \mathrm{H}), 6.99(\mathrm{dd}, J=8.7,8.7 \mathrm{~Hz}, 2 \mathrm{H}), 6.34(\mathrm{~d}, J=15.5 \mathrm{~Hz}, 1 \mathrm{H}), 6.14(\mathrm{dt}, J=15.5,7.3 \mathrm{~Hz}$, $1 \mathrm{H}), 2.10(\mathrm{t}, J=7.0 \mathrm{~Hz}, 2 \mathrm{H}), 1.80-1.70(\mathrm{~m}, 1 \mathrm{H}), 0.96(\mathrm{~d}, J=6.7 \mathrm{~Hz}, 6 \mathrm{H}) \cdot{ }^{13} \mathrm{C}$ NMR $\left(126 \mathrm{MHz}, \mathrm{CDCl}_{3}\right) \delta$ 162.7, 160.8, 134.0, 129.5, $127.2(\mathrm{~d}, J=7.8 \mathrm{~Hz}), 115.2(\mathrm{~d}, J=21.5 \mathrm{~Hz}), 42.2,28.5,22.3 .{ }^{19} \mathrm{~F} \mathrm{NMR}(471 \mathrm{MHz}$, $\left.\mathrm{CDCl}_{3}\right) \delta$ 115.94. IR (neat, $\mathrm{cm}^{-1}$ ): 2956, 2927, 2870, 1602, 1508, 1465, 1436, 1384, 1367, 1227, 1157, 1093, 966, 859, 841, 829, 780, 766, 573, 519. HRMS (EI) calcd for $\mathrm{C}_{12} \mathrm{H}_{15} \mathrm{~F}[\mathrm{M}]^{+} 178.1158$, found 178.1157 .

(E)-1,2,3-Trimethoxy-5-(4-methylpent-1-en-1-yl)benzene (2e)<smiles>COc1cc(/C=C/CC(C)C)cc(OC)c1OC</smiles>

Following general procedure GP1 using $(E)$-methyl (3-(3,4,5-trimethoxyphenyl)allyl) carbonate (84.7 mg, $0.3 \mathrm{mmol}, 1.0$ equiv), diethyl 4-isopropyl-2,6-dimethyl-1,4-dihydropyridine-3,5-dicarboxylate (146.3 $\mathrm{mg}$, $0.45 \mathrm{mmol}, 1.5$ equiv), (phen) $\mathrm{NiCl}_{2}(4.7 \mathrm{mg}, 5 \mathrm{~mol} \%$ ), and 4 CzIPN (7.1 mg, $3 \mathrm{~mol} \%$ ) in DMF ( $3 \mathrm{~mL}, 0.1$ $\mathrm{M})$. The reaction was stirred under blue LEDs at $\mathrm{rt}$ for $16 \mathrm{~h}$. Silica gel purification using an automated system (hexanes/EtOAc, 0 to $10 \%$ ) gave the product as a colorless oil $(47.3 \mathrm{mg}, 63 \%) .{ }^{1} \mathrm{H}$ NMR $\left(500 \mathrm{MHz}, \mathrm{CDCl}_{3}\right)$ $\delta 6.59(\mathrm{~s}, 2 \mathrm{H}), 6.30(\mathrm{~d}, J=15.7 \mathrm{~Hz}, 1 \mathrm{H}), 6.14(\mathrm{dt}, J=15.3,7.2 \mathrm{~Hz}, 1 \mathrm{H}), 3.89(\mathrm{~s}, 6 \mathrm{H}), 3.85(\mathrm{~s}, 3 \mathrm{H}), 2.15-$ $2.05(\mathrm{~m}, 2 \mathrm{H}), 1.74(\mathrm{~m}, 1 \mathrm{H}), 0.96(\mathrm{~d}, J=6.6 \mathrm{~Hz}, 6 \mathrm{H}) .{ }^{13} \mathrm{C} \mathrm{NMR}\left(126 \mathrm{MHz}, \mathrm{CDCl}_{3}\right) \delta 153.2,133.6,130.6$, 129.3, 125.2, 102.9, 60.8, 56.0, 42.3, 28.5, 22.3. IR (neat, $\mathrm{cm}^{-1}$ ): 2957, 2837, 2253, 1726, 1583, 1507, 1464, 1417,1384 1333, 1239, 1185, 1128, 1005, 965, 905, 828, 779, 726, 649. HRMS (EI) calcd for $\mathrm{C}_{15} \mathrm{H}_{22} \mathrm{O}_{3}[\mathrm{M}]^{+}$ 250.1569 , found 250.1560 .

(E)-(6,10-Dimethylundeca-4,9-dien-2-yl)benzene (2f) 


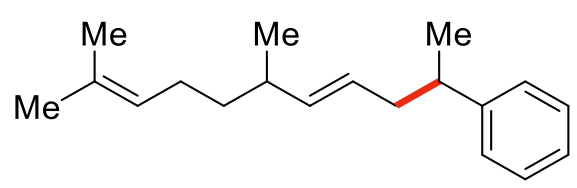

Following general procedure GP1 using (E)-4,8-dimethylnona-2,7-dien-1-yl methyl carbonate $(67.9 \mathrm{mg}, 0.3$ mmol, 1.0 equiv), diethyl 2,6-dimethyl-4-(1-phenylethyl)-1,4-dihydropyridine-3,5-dicarboxylate (160.8 mg, $0.45 \mathrm{mmol}, 1.5$ equiv), (phen) $\mathrm{NiCl}_{2}(4.7 \mathrm{mg}, 5 \mathrm{~mol} \%)$, and $4 \mathrm{CzIPN}(7.1 \mathrm{mg}, 3 \mathrm{~mol} \%)$ in DMF ( $3 \mathrm{~mL}, 0.1$ $\mathrm{M})$. The reaction was stirred under blue LEDs at $\mathrm{rt}$ for $16 \mathrm{~h}$. Silica gel purification using an automated system (hexanes/EtOAc, 0 to $10 \%$ ) gave the product as a colorless oil $(42.3 \mathrm{mg}, 55 \%) .{ }^{1} \mathrm{H} \mathrm{NMR}\left(500 \mathrm{MHz}, \mathrm{CDCl}_{3}\right)$ $\delta$ 7.31-7.28 (m, 2H), 7.22-7.13 (m, 3H), 5.42-5.28 (m, 2H), 5.11-5.07 (m, 1H), 2.77-2.72 (m, 1H), 2.36-2.30 $(\mathrm{m}, 1 \mathrm{H}), 2.26-2.18(\mathrm{~m}, 1 \mathrm{H}), 1.99-1.93(\mathrm{~m}, 3 \mathrm{H}), 1.82-1.78(\mathrm{~m}, 1 \mathrm{H}), 1.70(\mathrm{~m}, 3 \mathrm{H}), 1.61(\mathrm{~s}, 3 \mathrm{H}), 1.44-1.40(\mathrm{~m}$, $1 \mathrm{H}), 1.28-1.25(\mathrm{~m}, 3 \mathrm{H}), 0.89-0.79(\mathrm{~m}, 3 \mathrm{H}) .{ }^{13} \mathrm{C} \mathrm{NMR}\left(126 \mathrm{MHz}, \mathrm{CDCl}_{3}\right) \delta 130.7,129.8,129.8,128.4,128.3$, 127.9, 127.8, 127.2, 125.9, 125.1, 40.4, 40.1, 36.7, 32.9, 25.9, 25.7, 21.6, 19.5, 17.8. IR (neat, $\mathrm{cm}^{-1}$ ): 3028, $2962,2913,2252,1603,1494,1452,1376,1093,969,905,729,699,650,545$. HRMS (EI) calcd for $\mathrm{C}_{19} \mathrm{H}_{28}$ $[\mathrm{M}]^{+}$256.2191, found 256.2189.

(E)-(4,9-Dimethyldeca-1,8-dien-1-yl)benzene (2g)<smiles>CC(C)=CCCC(C)CC=Cc1ccccc1</smiles>

Following general procedure GP1 using cinnamyl methyl carbonate ( $57.7 \mathrm{mg}, 0.3 \mathrm{mmol}, 1.0$ equiv), diethyl 2,6-dimethyl-4-(6-methylhept-5-en-2-yl)-1,4-dihydropyridine-3,5-dicarboxylate (163.6 mg, $0.45 \mathrm{mmol}, 1.5$ equiv), (phen) $\mathrm{NiCl}_{2}(4.7 \mathrm{mg}, 5 \mathrm{~mol} \%)$, and $4 \mathrm{CzIPN}$ ( $7.1 \mathrm{mg}, 3 \mathrm{~mol} \%$ ) in DMF ( $\left.3 \mathrm{~mL}, 0.1 \mathrm{M}\right)$. The reaction was stirred under blue LEDs at $\mathrm{rt}$ for $16 \mathrm{~h}$. Silica gel purification using an automated system (hexanes/EtOAc, 0 to $10 \%)$ gave the product as a colorless oil $(48.6 \mathrm{mg}, 67 \%) .{ }^{1} \mathrm{H}$ NMR $\left(500 \mathrm{MHz}, \mathrm{CDCl}_{3}\right) \delta 7.36(\mathrm{~d}, J=7.4$ $\mathrm{Hz}, 2 \mathrm{H}), 7.31(\mathrm{dd}, J=7.6,7.6 \mathrm{~Hz}, 2 \mathrm{H}), 7.20(\mathrm{t}, J=7.3 \mathrm{~Hz}, 1 \mathrm{H}), 6.39(\mathrm{~d}, J=15.8 \mathrm{~Hz}, 1 \mathrm{H}), 6.23(\mathrm{dt}, J=15.8$, $7.2 \mathrm{~Hz}, 1 \mathrm{H}), 5.13(\mathrm{t}, J=7.1 \mathrm{~Hz}, 1 \mathrm{H}), 2.28-2.21(\mathrm{~m}, 1 \mathrm{H}), 2.10-1.96(\mathrm{~m}, 3 \mathrm{H}), 1.71(\mathrm{~s}, 3 \mathrm{H}), 1.63(\mathrm{~s}, 3 \mathrm{H}), 1.62$ $-1.57(\mathrm{~m}, 1 \mathrm{H}), 1.46-1.37(\mathrm{~m}, 1 \mathrm{H}), 1.25-1.18(\mathrm{~m}, 1 \mathrm{H}), 0.95(\mathrm{~d}, J=6.5 \mathrm{~Hz}, 3 \mathrm{H}) .{ }^{13} \mathrm{C} \mathrm{NMR}(126 \mathrm{MHz}$, $\left.\mathrm{CDCl}_{3}\right) \delta 137.8,131.1,130.8,129.6,128.4,126.7,125.8,124.7,40.4,36.6,32.8,25.6,25.5,19.4,17.6 . \mathrm{IR}$ (neat, $\mathrm{cm}^{-1}$ ): 3026, 2963, 2911, 1652, 1599, 1494, 1377, 1115, 1071, 1028, 964, 909, 829, 738, 691, 617, 524, 494. HRMS (EI) calcd for $\mathrm{C}_{17} \mathrm{H}_{24}[\mathrm{M}]^{+} 228.1878$, found 228.1832 .

Cinnamylcycloheptane (2h)

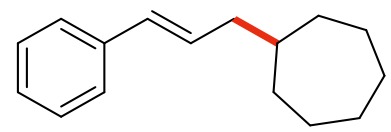

Following general procedure GP1 using cinnamyl methyl carbonate $(57.7 \mathrm{mg}, 0.3 \mathrm{mmol}, 1.0$ equiv), diethyl 4-cycloheptyl-2,6-dimethyl-1,4-dihydropyridine-3,5-dicarboxylate (170.8 mg, $0.45 \mathrm{mmol}, 1.5$ equiv), (phen) $\mathrm{NiCl}_{2}$ (4.7 mg, $5 \mathrm{~mol} \%$ ), and 4CzIPN (7.1 mg, $3 \mathrm{~mol} \%$ ) in DMF (3 mL, $\left.0.1 \mathrm{M}\right)$. The reaction was stirred under blue LEDs at $\mathrm{rt}$ for $16 \mathrm{~h}$. Silica gel purification using an automated system (hexanes/EtOAc, 0 to $10 \%)$ gave the product as a colorless oil $(46.2 \mathrm{mg}, 72 \%) .{ }^{1} \mathrm{H}$ NMR $\left(500 \mathrm{MHz}, \mathrm{CDCl}_{3}\right) \delta 7.35(\mathrm{~d}, J=7.6$ $\mathrm{Hz}, 2 \mathrm{H}), 7.29$ (dd, $J=7.6,7.3 \mathrm{~Hz}, 2 \mathrm{H}), 7.19(\mathrm{t}, J=7.3 \mathrm{~Hz}, 1 \mathrm{H}), 6.36(\mathrm{~d}, J=15.8 \mathrm{~Hz}, 1 \mathrm{H}), 6.21$ (dt, $J=15.4$, $7.1 \mathrm{~Hz}, 1 \mathrm{H}), 2.14(\mathrm{t}, J=7.0 \mathrm{~Hz}, 2 \mathrm{H}), 1.79-1.73(\mathrm{~m}, 2 \mathrm{H}), 1.68-1.57(\mathrm{~m}, 5 \mathrm{H}), 1.52-1.39(\mathrm{~m}, 4 \mathrm{H}), 1.26-1.19(\mathrm{~m}$, $2 \mathrm{H}) .{ }^{13} \mathrm{C} \mathrm{NMR}\left(126 \mathrm{MHz}, \mathrm{CDCl}_{3}\right) \delta 138.1,130.9,130.4,128.6,126.9,126.0,41.9,39.9,34.6,28.6,26.7$. IR (neat, $\mathrm{cm}^{-1}$ ): 3025, 2917, 2851, 1650, 1620, 1599, 1495, 1459, 1444, 1360, 1325, 1269, 1213, 1086, 963, 820, 769, 743, 691, 497. HRMS (EI) calcd for $\mathrm{C}_{16} \mathrm{H}_{22}[\mathrm{M}]^{+}$214.1722, found 214.1724.

(E)-(4-(Benzyloxy)but-1-en-1-yl)benzene (2i) 


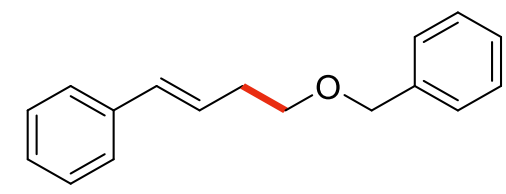

Following general procedure GP1 using cinnamyl methyl carbonate ( $57.7 \mathrm{mg}, 0.3 \mathrm{mmol}, 1.0$ equiv), diethyl 4-((benzyloxy)methyl)-2,6-dimethyl-1,4-dihydropyridine-3,5-dicarboxylate (186.7 mg, $0.45 \mathrm{mmol}, 1.5$ equiv), (phen) $\mathrm{NiCl}_{2}(4.7 \mathrm{mg}, 5 \mathrm{~mol} \%)$, and $4 \mathrm{CzIPN}$ ( $7.1 \mathrm{mg}, 3 \mathrm{~mol} \%$ ) in DMF ( $\left.3 \mathrm{~mL}, 0.1 \mathrm{M}\right)$. The reaction was stirred under blue LEDs at $\mathrm{rt}$ for $16 \mathrm{~h}$. Silica gel purification using an automated system (hexanes/EtOAc, 0 to $10 \%)$ gave the product as a colorless oil $(50.6 \mathrm{mg}, 71 \%) .{ }^{1} \mathrm{H} \mathrm{NMR}\left(500 \mathrm{MHz}, \mathrm{CDCl}_{3}\right) \delta 7.38-7.33(\mathrm{~m}$, $6 \mathrm{H}), 7.32-7.27(\mathrm{~m}, 3 \mathrm{H}), 7.24-7.17(\mathrm{~m}, 1 \mathrm{H}), 6.47(\mathrm{~d}, J=15.6 \mathrm{~Hz}, 1 \mathrm{H}), 6.25(\mathrm{dt}, J=15.9,7.0 \mathrm{~Hz}, 1 \mathrm{H}), 4.56$ $(\mathrm{s}, 2 \mathrm{H}), 3.61(\mathrm{t}, J=6.7 \mathrm{~Hz}, 2 \mathrm{H}), 2.55(\mathrm{ddd}, J=6.8,6.7,1.5 \mathrm{~Hz}, 2 \mathrm{H}) .{ }^{13} \mathrm{C} \mathrm{NMR}\left(126 \mathrm{MHz}, \mathrm{CDCl}_{3}\right) \delta 138.3$, 137.5, 131.5, 128.4, 128.3, 127.6, 127.5, 126.9, 125.9, 72.9, 69.7, 33.4. IR (neat, $\left.\mathrm{cm}^{-1}\right): 3061,3027,2854$, 1598, 1495, 1453, 1362, 1308, 1205, 1095, 1028, 964, 910, 844, 792, 734, 693, 606, 495.6, 460.3. HRMS (EI) calcd for $\mathrm{C}_{17} \mathrm{H}_{18} \mathrm{O}[\mathrm{M}]^{+} 238.1385$, found 238.1387.

(E)-2-Cinnamylbicyclo[2.2.1] heptane $^{8}(\mathbf{2 j})$<smiles>C(=Cc1ccccc1)CC1CC2CCC1C2</smiles>

Following general procedure GP1 using cinnamyl methyl carbonate ( $57.7 \mathrm{mg}, 0.3 \mathrm{mmol}, 1.0$ equiv), diethyl 4-((IR*,4S*)-bicyclo[2.2.1]heptan-2-yl)-2,6-dimethyl-1,4-dihydropyridine-3,5-dicarboxylate (172.7 $\mathrm{mg}$, $0.45 \mathrm{mmol}, 1.5$ equiv), (phen) $\mathrm{NiCl}_{2}(4.7 \mathrm{mg}, 5 \mathrm{~mol} \%)$, and $4 \mathrm{CzIPN}(7.1 \mathrm{mg}, 3 \mathrm{~mol} \%)$ in DMF (3 mL, 0.1 M). The reaction was stirred under blue LEDs at $\mathrm{rt}$ for $16 \mathrm{~h}$. Silica gel purification using an automated system (hexanes/EtOAc, 0 to $10 \%)$ gave the product as a colorless oil $(47.0 \mathrm{mg}, 74 \%) .{ }^{1} \mathrm{H} \mathrm{NMR}\left(500 \mathrm{MHz}, \mathrm{CDCl}_{3}\right)$ $\delta 7.38-7.33(\mathrm{~m}, 2 \mathrm{H}), 7.29(\mathrm{dd}, J=7.7,7.1 \mathrm{~Hz}, 2 \mathrm{H}), 7.19(\mathrm{td}, J=7.1,1.5 \mathrm{~Hz}, 1 \mathrm{H}), 6.36(\mathrm{~d}, J=15.7 \mathrm{~Hz}, 1 \mathrm{H})$, 6.19 (dt, $J=15.7,7.0 \mathrm{~Hz}, 1 \mathrm{H}), 2.24(\mathrm{~s}, 1 \mathrm{H}), 2.21-2.15(\mathrm{~m}, 1 \mathrm{H}), 2.06-2.01(\mathrm{~m}, 2 \mathrm{H}), 1.60-1.43(\mathrm{~m}, 4 \mathrm{H}), 1.36$ $(\mathrm{dt}, J=9.7,2.0 \mathrm{~Hz}, 1 \mathrm{H}), 1.21-1.10(\mathrm{~m}, 4 \mathrm{H}) .{ }^{13} \mathrm{C} \mathrm{NMR}\left(126 \mathrm{MHz}, \mathrm{CDCl}_{3}\right) \delta 137.9,130.2,130.0,128.4,126.6$, 125.8, 42.0, 40.6, 40.2, 37.8, 36.6, 35.1, 30.0, 28.8. IR (neat, $\mathrm{cm}^{-1}$ ): 3059, 3025, 2947, 2912, 2868, 1651, $1599,1493,1450,1434,1313,1139,1072,1029,964,791,748,726,691,522$. HRMS (EI) calcd for $\mathrm{C}_{16} \mathrm{H}_{20}$ $[\mathrm{M}]^{+} 212.1565$, found 212.1567 .

\subsection{Reactions Using Allylic Alcohols.}

\subsubsection{General Procedure (GP2)}

To an $8-\mathrm{mL}$ reaction vial equipped with a stir bar and septa screw cap were added alkyl 1,4-dihydropyridines ( $0.45 \mathrm{mmol}, 1.5$ equiv), allylic alcohols ( $0.3 \mathrm{mmol}, 1.0$ equiv), DMDC (0.9 mmol. 3.0 equiv), (phen) $\mathrm{NiCl}_{2}$ $(4.7 \mathrm{mg}, 5 \mathrm{~mol} \%)$, and $4 \mathrm{CzIPN}(7.1 \mathrm{mg}, 3 \mathrm{~mol} \%)$. The reaction vial was subsequently closed, and three vacuum/argon cycles were performed, followed by addition of $3 \mathrm{~mL}$ of dry $\operatorname{DMF}(0.1 \mathrm{M})$. After stirring under blue LEDs for $16 \mathrm{~h}$, the reaction was diluted with $30 \mathrm{~mL}$ of EtOAc and washed three times with $10 \mathrm{~mL}$ of saturated $\mathrm{Na}_{2} \mathrm{CO}_{3}$ and subsequently three times with $10 \mathrm{~mL}$ of brine. The organic phase was dried $\left(\mathrm{MgSO}_{4}\right)$, and the solvent was removed by rotovap. The final product was purified by flash column chromatography, using hexanes and EtOAc as eluent.

\subsubsection{Characterization Data}

(E)-1-(3-Cyclohexylprop-1-en-1-yl)-3,5-difluorobenzene (3c)<smiles>Fc1cc(F)cc(C=CCC2CCCCC2)c1</smiles>

Following general procedure GP2 using (E)-3-(3,5-difluorophenyl)prop-2-en-1-ol (51.1 mg, $0.3 \mathrm{mmol}, 1.0$ equiv), diethyl 4-cyclohexyl-2,6-dimethyl-1,4-dihydropyridine-3,5-dicarboxylate (150.9 mg, $0.45 \mathrm{mmol}, 1.5$ 
equiv), DMDC (127 mg, $0.9 \mathrm{mmol}, 3.0$ equiv), (phen) $\mathrm{NiCl}_{2}$ (4.7 mg, $5 \mathrm{~mol} \%$ ), and 4CzIPN (7.1 mg, $3 \mathrm{~mol}$ $\%$ ) in DMF (3 mL, 0.1 M). The reaction was stirred under blue LEDs at $\mathrm{rt}$ for $16 \mathrm{~h}$. Silica gel purification using an automated system (hexanes/EtOAc, 0 to $10 \%)$ gave the product as a colorless oil $(29.3 \mathrm{mg}, 41 \%)$. ${ }^{1} \mathrm{H}$ NMR $\left(500 \mathrm{MHz}, \mathrm{CDCl}_{3}\right) \delta 7.40-7.36(\mathrm{~m}, 1 \mathrm{H}), 6.82-6.74(\mathrm{~m}, 2 \mathrm{H}), 6.42(\mathrm{~d}, J=15.9 \mathrm{~Hz}, 1 \mathrm{H}), 6.21(\mathrm{dt}$, $J=15.4,7.3 \mathrm{~Hz}, 1 \mathrm{H}), 2.11(\mathrm{t}, J=7.0 \mathrm{~Hz}, 2 \mathrm{H}), 1.79-1.68(\mathrm{~m}, 4 \mathrm{H}), 1.68-1.62(\mathrm{~m}, 1 \mathrm{H}), 1.43-1.36(\mathrm{~m}, 1 \mathrm{H})$, $1.25-1.14(\mathrm{~m}, 3 \mathrm{H}), 0.99-0.92(\mathrm{~m}, 2 \mathrm{H}) .{ }^{13} \mathrm{C}$ NMR $\left(126 \mathrm{MHz}, \mathrm{CDCl}_{3}\right) \delta 160.8,132.3(\mathrm{~d}, J=4.1 \mathrm{~Hz}), 127.9$ $(\mathrm{dd}, J=9.3,5.7 \mathrm{~Hz}), 122.2,111.4(\mathrm{~d}, J=24.8 \mathrm{~Hz}), 104.0(\mathrm{t}, J=25.7 \mathrm{~Hz}), 41.3,38.0,33.1,26.4,26.2 .{ }^{19} \mathrm{~F}$ NMR $\left(471 \mathrm{MHz}, \mathrm{CDCl}_{3}\right) \delta-112.68(\mathrm{~d}, J=6.7 \mathrm{~Hz}),-114.88(\mathrm{~d}, J=6.8 \mathrm{~Hz})$. IR (neat, $\left.\mathrm{cm}^{-1}\right): 2922,2852,1613$, $1593,1500,1449,1428,1274,1138,1090,965,941,848,807,728,608,535,528,505,498$. HRMS (EI) calcd for $\mathrm{C}_{15} \mathrm{H}_{18} \mathrm{~F}_{2}[\mathrm{M}]^{+} 236.1377$, found 236.1368 .

(E)-1-Chloro-4-(4-methylpent-1-en-1-yl)benzene (3d)<smiles>CC(C)C/C=C/c1ccc(Cl)cc1</smiles>

Following general procedure GP2 using (E)-3-(4-chlorophenyl)prop-2-en-1-ol (50.6 mg, $0.3 \mathrm{mmol}, 1.0$ equiv), diethyl 4-isopropyl-2,6-dimethyl-1,4-dihydropyridine-3,5-dicarboxylate (146.3 mg, $0.45 \mathrm{mmol}, 1.5$ equiv), DMDC (127 mg, $0.9 \mathrm{mmol}, 3.0$ equiv), (phen) $\mathrm{NiCl}_{2}$ ( $4.7 \mathrm{mg}, 5 \mathrm{~mol} \%$ ), and 4CzIPN (7.1 mg, $3 \mathrm{~mol} \%$ ) in DMF ( $3 \mathrm{~mL}, 0.1 \mathrm{M})$. The reaction was stirred under blue LEDs at $\mathrm{rt}$ for $16 \mathrm{~h}$. Silica gel purification using an automated system (hexanes/EtOAc, 0 to $10 \%$ ) gave the product as a colorless oil (40.9 mg, 70\%). ${ }^{1} \mathrm{H} \mathrm{NMR}$ $\left(500 \mathrm{MHz}, \mathrm{CDCl}_{3}\right) \delta 7.29-7.25(\mathrm{~m}, 4 \mathrm{H}), 6.33(\mathrm{~d}, J=15.5 \mathrm{~Hz}, 1 \mathrm{H}), 6.20(\mathrm{dt}, J=15.3,7.1 \mathrm{~Hz}, 1 \mathrm{H}), 2.10$ $(\mathrm{ddd}, J=7.1,7.1,1.2 \mathrm{~Hz}, 2 \mathrm{H}), 1.77-1.72(\mathrm{~m}, 1 \mathrm{H}), 0.95(\mathrm{~d}, J=6.7 \mathrm{~Hz}, 6 \mathrm{H}) .{ }^{13} \mathrm{C} \mathrm{NMR}\left(126 \mathrm{MHz}, \mathrm{CDCl}_{3}\right) \delta$ 136.3, 132.2, 130.6, 129.5, 128.5, 127.0, 42.3, 28.5, 22.3. IR (neat, $\mathrm{cm}^{-1}$ ): 2955, 2927, 2869, 1711, 1491, 1464, 1404 1384, 1366, 1220, 1091, 1012, 966, 856, 827, 799, 777, 679, 528, 502. HRMS (EI) calcd for $\mathrm{C}_{12} \mathrm{H}_{15} \mathrm{Cl}[\mathrm{M}]^{+}$194.0862, found 194.0861.

(E)-1-(4-Methylpent-1-en-1-yl)-4-phenoxybenzene (3e)<smiles>CC(C)C/C=C/c1ccc(Oc2ccccc2)cc1</smiles>

Following general procedure GP2 using (E)-3-(4-phenoxyphenyl)prop-2-en-1-ol (67.9 mg, $0.3 \mathrm{mmol}, 1.0$ equiv), diethyl 4-isopropyl-2,6-dimethyl-1,4-dihydropyridine-3,5-dicarboxylate (146.3 mg, $0.45 \mathrm{mmol}, 1.5$ equiv), DMDC (127 mg, $0.9 \mathrm{mmol}, 3.0$ equiv), (phen) $\mathrm{NiCl}_{2}$ (4.7 mg, $5 \mathrm{~mol} \%$ ), and 4CzIPN (7.1 mg, $3 \mathrm{~mol} \%$ ) in DMF ( $3 \mathrm{~mL}, 0.1 \mathrm{M})$. The reaction was stirred under blue LEDs at $\mathrm{rt}$ for $16 \mathrm{~h}$. Silica gel purification using an automated system (hexanes/EtOAc, 0 to $10 \%$ ) gave the product as a colorless oil (48.4 mg, 64\%). ${ }^{1} \mathrm{H} \mathrm{NMR}$ $\left(500 \mathrm{MHz}, \mathrm{CDCl}_{3}\right) \delta 7.35-7.32(\mathrm{~m}, 4 \mathrm{H}), 7.10(\mathrm{t}, J=6.8 \mathrm{~Hz}, 1 \mathrm{H}), 7.01(\mathrm{~d}, J=8.6 \mathrm{~Hz}, 2 \mathrm{H}), 6.96(\mathrm{~d}, J=8.6$ $\mathrm{Hz}, 2 \mathrm{H}), 6.35(\mathrm{~d}, J=15.5 \mathrm{~Hz}, 1 \mathrm{H}), 6.15(\mathrm{dt}, J=15.1,7.0 \mathrm{~Hz}, 1 \mathrm{H}), 2.10(\mathrm{ddd}, J=6.9,6.8,1.2 \mathrm{~Hz}, 2 \mathrm{H}), 1.76$ $-1.71(\mathrm{~m}, 1 \mathrm{H}), 0.95(\mathrm{~d}, J=6.5 \mathrm{~Hz}, 6 \mathrm{H}) .{ }^{13} \mathrm{C} \mathrm{NMR}\left(126 \mathrm{MHz}, \mathrm{CDCl}_{3}\right) \delta 156.1,133.5,130.1,129.8,129.2$, 127.3, 123.2, 119.2, 118.8, 118.6, 42.5, 28.8, 22.5. IR (neat, $\mathrm{cm}^{-1}$ ): 2981, 2254, 1710, 1419, 1361, 1222, 1091, 910, 727, 648, 530. HRMS (EI) calcd for $\mathrm{C}_{18} \mathrm{H}_{20} \mathrm{O}[\mathrm{M}]^{+} 252.1519$, found 252.1506.

(E)-6-(4-Methylpent-1-en-1-yl)-2,3-dihydrobenzo[b][1,4]dioxin (3f)<smiles>CC(C)C/C=C/c1ccc2c(c1)OCCO2</smiles>

Following general procedure GP2 using (E)-3-(2,3-dihydrobenzo[b][1,4]dioxin-6-yl)prop-2-en-1-ol (57.7 mg, $0.3 \mathrm{mmol}, 1.0$ equiv), diethyl 4-isopropyl-2,6-dimethyl-1,4-dihydropyridine-3,5-dicarboxylate (146.3 mg, 
$0.45 \mathrm{mmol}, 1.5$ equiv), DMDC (127 mg, $0.9 \mathrm{mmol}, 3.0$ equiv), (phen) $\mathrm{NiCl}_{2}$ (4.7 $\mathrm{mg}, 5 \mathrm{~mol} \%$ ), and 4CzIPN (7.1 mg, $3 \mathrm{~mol} \%$ ) in DMF (3 mL, $0.1 \mathrm{M})$. The reaction was stirred under blue LEDs at $\mathrm{rt}$ for $16 \mathrm{~h}$. Silica gel purification using an automated system (hexanes/EtOAc, 0 to 10\%) gave the product as a colorless oil (46.9 mg, 72\%). ${ }^{1} \mathrm{H}$ NMR $\left(500 \mathrm{MHz}, \mathrm{CDCl}_{3}\right) \delta 6.88(\mathrm{~d}, J=2.0 \mathrm{~Hz}, 1 \mathrm{H}), 6.84(\mathrm{dd}, J=8.3,2.1 \mathrm{~Hz}, 1 \mathrm{H}), 6.79(\mathrm{~d}, J$ $=8.4 \mathrm{~Hz}, 1 \mathrm{H}), 6.25(\mathrm{~d}, J=15.6 \mathrm{~Hz}, 1 \mathrm{H}), 6.07(\mathrm{dt}, J=15.6,7.3 \mathrm{~Hz}, 1 \mathrm{H}), 4.26(\mathrm{~s}, 4 \mathrm{H}), 2.07(\mathrm{ddd}, J=7.4,7.0$, $1.1 \mathrm{~Hz}, 2 \mathrm{H}), 1.75-1.67(\mathrm{~m}, 1 \mathrm{H}), 0.93(\mathrm{~d}, J=6.6 \mathrm{~Hz}, 6 \mathrm{H}) .{ }^{13} \mathrm{C} \mathrm{NMR}\left(126 \mathrm{MHz}, \mathrm{CDCl}_{3}\right) \delta 143.3,142.5,131.8$, 129.9, 128.2, 119.2, 117.1, 114.3, 64.3, 64.3 42.2, 28.5, 22.3. IR (neat, $\left.\mathrm{cm}^{-1}\right): 3416,3004,1710,1583,1508$, $1421,1359,1307,1286,1258,1220,1092,1069,970,890,818,784,529$. HRMS (EI) calcd for $\mathrm{C}_{14} \mathrm{H}_{18} \mathrm{O}_{2}$ $[\mathrm{M}]^{+}$218.1307, found 218.1298.

(E)-1-Methoxy-4-(4-methylpent-1-en-1-yl)benzene (3g)<smiles>COc1ccc(/C=C/CC(C)C)cc1</smiles>

Following general procedure GP2 using (E)-3-(4-methoxyphenyl)prop-2-en-1-ol (49.3 mg, $0.3 \mathrm{mmol}, 1.0$ equiv), diethyl 4-isopropyl-2,6-dimethyl-1,4-dihydropyridine-3,5-dicarboxylate (146.3 $\mathrm{mg}, 0.45 \mathrm{mmol}, 1.5$ equiv), DMDC (127 mg, $0.9 \mathrm{mmol}, 3.0$ equiv), (phen)NiCl 2 ( $4.7 \mathrm{mg}, 5 \mathrm{~mol} \%$ ), and 4CzIPN (7.1 mg, $3 \mathrm{~mol} \%$ ) in DMF ( $3 \mathrm{~mL}, 0.1 \mathrm{M})$. The reaction was stirred under blue LEDs at $\mathrm{rt}$ for $16 \mathrm{~h}$. Silica gel purification using an automated system (hexanes/EtOAc, 0 to $10 \%$ ) gave the product as a colorless oil (43.5 mg, 76\%). ${ }^{1} \mathrm{H} \mathrm{NMR}$ $\left(500 \mathrm{MHz}, \mathrm{CDCl}_{3}\right) \delta 7.30(\mathrm{~d}, J=8.7 \mathrm{~Hz}, 2 \mathrm{H}), 6.85(\mathrm{~d}, J=8.6 \mathrm{~Hz}, 2 \mathrm{H}), 6.33(\mathrm{~d}, J=15.9 \mathrm{~Hz}, 1 \mathrm{H}), 6.09(\mathrm{dt}, J$ $=15.4,7.3 \mathrm{~Hz}, 1 \mathrm{H}), 3.82(\mathrm{~s}, 3 \mathrm{H}), 2.09(\mathrm{dd}, J=6.9,6.8 \mathrm{~Hz}, 2 \mathrm{H}), 1.78-1.69(\mathrm{~m}, 1 \mathrm{H}), 0.95(\mathrm{~d}, J=6.5 \mathrm{~Hz}, 6 \mathrm{H})$. ${ }^{13} \mathrm{C}$ NMR $\left(126 \mathrm{MHz}, \mathrm{CDCl}_{3}\right) \delta 158.5,130.7,130.0,127.6,126.9,113.8,55.2,42.3,28.6,22.3$. IR (neat, $\mathrm{cm}^{-}$ $\left.{ }^{1}\right)$ : 2969, 2930, 1530, 1511, 1454, 1411, 1377, 1357, 1328, 1273, 1236, 1216, 1140, 1110, 1076, 1048, 971, 815, 783, 583. HRMS (EI) calcd for $\mathrm{C}_{13} \mathrm{H}_{18} \mathrm{O}[\mathrm{M}]^{+}$190.1358, found 190.1346 .

(E)-(3-(Cyclohex-3-en-1-yl)prop-1-en-1-yl)benzene (3h)<smiles>C1=CCC(C/C=C/c2ccccc2)CC1</smiles>

Following general procedure GP2 using (E)-3-phenylprop-2-en-1-ol (40.3 mg, $0.3 \mathrm{mmol}, 1.0$ equiv), diethyl 4-(cyclohex-3-en-1-yl)-2,6-dimethyl-1,4-dihydropyridine-3,5-dicarboxylate $(150.0 \mathrm{mg}, 0.45 \mathrm{mmol}, 1.5$ equiv), DMDC (127 mg, $0.9 \mathrm{mmol}, 3.0$ equiv), (phen) $\mathrm{NiCl}_{2}$ ( $4.7 \mathrm{mg}, 5 \mathrm{~mol} \%$ ), and 4CzIPN (7.1 mg, $3 \mathrm{~mol} \%$ ) in DMF ( $3 \mathrm{~mL}, 0.1 \mathrm{M})$. The reaction was stirred under blue LEDs at $\mathrm{rt}$ for $16 \mathrm{~h}$. Silica gel purification using an automated system (hexanes/EtOAc, 0 to $10 \%$ ) gave the product as a colorless oil ( $35.5 \mathrm{mg}, 60 \%) .{ }^{1} \mathrm{H}$ NMR $\left(500 \mathrm{MHz}, \mathrm{CDCl}_{3}\right) \delta 7.38-7.34(\mathrm{~m}, 2 \mathrm{H}), 7.29(\mathrm{dd}, J=7.7,7.7 \mathrm{~Hz}, 2 \mathrm{H}), 7.20(\mathrm{td}, J=7.0,1.5 \mathrm{~Hz}, 1 \mathrm{H}), 6.40$ $(\mathrm{dd}, J=15.9,4.2 \mathrm{~Hz}, 1 \mathrm{H}), 6.24(\mathrm{dt}, J=15.7,7.2 \mathrm{~Hz}, 1 \mathrm{H}), 5.73-5.64(\mathrm{~m}, 2 \mathrm{H}), 2.25-2.19(\mathrm{~m}, 2 \mathrm{H}), 2.18-$ $2.11(\mathrm{~m}, 1 \mathrm{H}), 2.09-1.99(\mathrm{~m}, 2 \mathrm{H}), 1.84-1.80(\mathrm{~m}, 1 \mathrm{H}), 1.78-1.70(\mathrm{~m}, 2 \mathrm{H}), 1.34-1.26(\mathrm{~m}, 1 \mathrm{H}) .{ }^{13} \mathrm{C} \mathrm{NMR}$ $\left(126 \mathrm{MHz}, \mathrm{CDCl}_{3}\right) \delta 137.7,130.9,129.2,128.4,126.9,126.7,126.3,125.8,39.9,33.8,31.6,28.4,25.0 . \mathrm{IR}$ $\left(\right.$ neat, $\left.\mathrm{cm}^{-1}\right)$ : 2969, 2930, 1530, 1511, 1454, 1411, 1377, 1357, 1328, 1273, 1236, 1216, 1140, 1110, 1076, 1048, 971, 815, 783, 583. HRMS (EI) calcd for $\mathrm{C}_{15} \mathrm{H}_{18}[\mathrm{M}]^{+}$198.1409, found 198.1403.

4-Cinnamyltetrahydro-2H-pyran (3i)

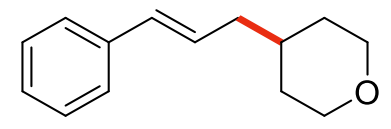

Following general procedure GP2 using (E)-3-phenylprop-2-en-1-ol (40.3 mg, $0.3 \mathrm{mmol}, 1.0$ equiv), diethyl 2,6-dimethyl-4-(tetrahydro-2H-pyran-4-yl)-1,4-dihydropyridine-3,5-dicarboxylate (151.8 mg, $0.45 \mathrm{mmol}$, 
1.5 equiv), DMDC (127 mg, $0.9 \mathrm{mmol}, 3.0$ equiv), (phen) $\mathrm{NiCl}_{2}(4.7 \mathrm{mg}, 5 \mathrm{~mol} \%$ ), and 4CzIPN (7.1 mg, 3 mol \%) in DMF ( $3 \mathrm{~mL}, 0.1 \mathrm{M})$. The reaction was stirred under blue LEDs at $\mathrm{rt}$ for $16 \mathrm{~h}$. Silica gel purification using an automated system (hexanes/EtOAc, 0 to $10 \%)$ gave the product as a colorless oil $(31.6 \mathrm{mg}, 52 \%)$. ${ }^{1} \mathrm{H}$ NMR $\left(500 \mathrm{MHz}, \mathrm{CDCl}_{3}\right) \delta 7.34(\mathrm{~d}, J=7.1 \mathrm{~Hz}, 2 \mathrm{H}), 7.30(\mathrm{dd}, J=7.6,7.6 \mathrm{~Hz}, 2 \mathrm{H}), 7.20(\mathrm{t}, J=7.2 \mathrm{~Hz}$, $1 \mathrm{H}), 6.39(\mathrm{~d}, J=15.8 \mathrm{~Hz}, 1 \mathrm{H}), 6.20(\mathrm{dt}, J=15.4,7.3 \mathrm{~Hz}, 1 \mathrm{H}), 3.99(\mathrm{dd}, J=11.5,4.5 \mathrm{~Hz}, 2 \mathrm{H}), 3.39(\mathrm{td}, J=$ $11.9,1.9 \mathrm{~Hz}, 2 \mathrm{H}), 2.20-2.14(\mathrm{~m}, 2 \mathrm{H}), 1.68-1.62(\mathrm{~m}, 3 \mathrm{H}), 1.40-1.32(\mathrm{~m}, 2 \mathrm{H}) .{ }^{13} \mathrm{C} \mathrm{NMR}\left(126 \mathrm{MHz}, \mathrm{CDCl}_{3}\right)$ $\delta 137.5,131.4,128.4,128.2,126.9,125.9,68.0,40.3,35.4,32.9$. IR (neat, $\mathrm{cm}^{-1}$ ): $3025,2951,2912,2838$, 1494, 1443, 1386, 1364, 1231, 1135, 1092, 1073, 1012, 982, 965, 891, 859, 742, 693. HRMS (EI) calcd for $\mathrm{C}_{14} \mathrm{H}_{18} \mathrm{O}[\mathrm{M}]^{+} 202.1358$, found 202.1359.

(E)-(4-Methylhex-1-en-1-yl)benzene (3j)<smiles>CCC(C)CC=Cc1ccccc1</smiles>

Following general procedure GP2 using (E)-3-phenylprop-2-en-1-ol (40.3 mg, $0.3 \mathrm{mmol}, 1.0$ equiv), diethyl 4-(sec-butyl)-2,6-dimethyl-1,4-dihydropyridine-3,5-dicarboxylate (139.2 mg, $0.45 \mathrm{mmol}, 1.5$ equiv), DMDC (127 mg, $0.9 \mathrm{mmol}, 3.0$ equiv), (phen) $\mathrm{NiCl}_{2}$ (4.7 mg, $5 \mathrm{~mol} \%$ ), and 4CzIPN (7.1 mg, $3 \mathrm{~mol} \%$ ) in DMF (3 $\mathrm{mL}, 0.1 \mathrm{M})$. The reaction was stirred under blue LEDs at $\mathrm{rt}$ for $16 \mathrm{~h}$. Silica gel purification using an automated system (hexanes/EtOAc, 0 to $10 \%)$ gave the product as a colorless oil $(41.8 \mathrm{mg}, 80 \%) .{ }^{1} \mathrm{H}$ NMR $(500 \mathrm{MHz}$, $\left.\mathrm{CDCl}_{3}\right) \delta 7.37-7.32(\mathrm{~m}, 2 \mathrm{H}), 7.29(\mathrm{t}, J=7.7 \mathrm{~Hz}, 2 \mathrm{H}), 7.21-7.16(\mathrm{~m}, 1 \mathrm{H}), 6.37(\mathrm{~d}, J=15.8 \mathrm{~Hz}, 1 \mathrm{H}), 6.21$ $(\mathrm{dt}, J=15.7,7.3 \mathrm{~Hz}, 1 \mathrm{H}), 2.26(\mathrm{~m}, 1 \mathrm{H}), 2.09-2.03(\mathrm{~m}, 1 \mathrm{H}), 1.53-1.49(\mathrm{~m}, 1 \mathrm{H}), 1.46-1.39(\mathrm{~m}, 1 \mathrm{H}), 1.24$ - $1.67(\mathrm{~m}, 1 \mathrm{H}), 0.94-0.90(\mathrm{~m}, 6 \mathrm{H}) .{ }^{13} \mathrm{C} \mathrm{NMR}\left(126 \mathrm{MHz}, \mathrm{CDCl}_{3}\right) \delta 137.9,130.7,129.7,128.3,126.6,125.8$, 40.1, 34.9, 29.1, 19.1, 11.4. IR (neat, $\mathrm{cm}^{-1}$ ): 2968, 2920, 1654, 1530, 1511, 1457, 1401, 1329, 1273, 1236, 1216, 1140, 1110, 1076, 971, 909, 829, 738, 692, 524. HRMS (EI) calcd for $\mathrm{C}_{13} \mathrm{H}_{18}[\mathrm{M}]^{+}$174.1409, found 174.1411.

Benzyl (E)-4-(3-(4-Methoxyphenyl)allyl)piperidine-1-carboxylate (3k)<smiles>COc1ccc(/C=C/CC2CCN(C(=O)OCc3ccccc3)CC2)cc1</smiles>

Following general procedure GP2 using (E)-3-(4-methoxyphenyl)prop-2-en-1-ol (49.3 mg, $0.3 \mathrm{mmol}, 1.0$ equiv), diethyl 4-isopropyl-2,6-dimethyl-1,4-dihydropyridine-3,5-dicarboxylate (211.8 mg, $0.45 \mathrm{mmol}, 1.5$ equiv), DMDC (127 mg, $0.9 \mathrm{mmol}, 3.0$ equiv), (phen) $\mathrm{NiCl}_{2}$ ( $4.7 \mathrm{mg}, 5 \mathrm{~mol} \%$ ), and 4CzIPN (7.1 mg, $3 \mathrm{~mol} \%$ ) in DMF ( $3 \mathrm{~mL}, 0.1 \mathrm{M})$. The reaction was stirred under blue LEDs at $\mathrm{rt}$ for $16 \mathrm{~h}$. Silica gel purification using an automated system (hexanes/EtOAc, 0 to $10 \%$ ) gave the product as a colorless oil (72.4 mg, 66\%). ${ }^{1} \mathrm{H} \mathrm{NMR}$ $\left(500 \mathrm{MHz}, \mathrm{CDCl}_{3}\right) \delta 7.38-7.33(\mathrm{~m}, 4 \mathrm{H}), 7.31(\mathrm{dd}, J=8.8,4.3 \mathrm{~Hz}, 1 \mathrm{H}), 7.29-7.27(\mathrm{~m}, 2 \mathrm{H}), 6.84(\mathrm{~d}, J=8.7$ $\mathrm{Hz}, 2 \mathrm{H}), 6.32(\mathrm{~d}, J=15.7 \mathrm{~Hz}, 1 \mathrm{H}), 6.03(\mathrm{dt}, J=15.4,7.3 \mathrm{~Hz}, 1 \mathrm{H}), 5.13(\mathrm{~s}, 2 \mathrm{H}), 4.18-4.10(\mathrm{~m}, 2 \mathrm{H}), 3.80(\mathrm{~s}$, $3 \mathrm{H}), 2.77(\mathrm{~s}, 2 \mathrm{H}), 2.14(\mathrm{dd}, J=7.0,6.9 \mathrm{~Hz}, 2 \mathrm{H}), 1.72(\mathrm{~s}, 2 \mathrm{H}), 1.28-1.25(\mathrm{~m}, 1 \mathrm{H}), 1.22-1.20(\mathrm{~m}, 2 \mathrm{H}) .{ }^{13} \mathrm{C}$ NMR $\left(126 \mathrm{MHz}, \mathrm{CDCl}_{3}\right) \delta 159.0,155.5,137.2,131.1,130.5,128.6,128.0,128.0,127.2,126.1,114.1,67.1$, 55.5, 44.4, 40.1, 36.6, 14.4. IR (neat, $\mathrm{cm}^{-1}$ ): 2929, 2852, 1694, 1606, 1511, 1466, 1364, 1276, 1244, 1175, 1128, 1108, 1087, 1030, 966, 830, 764, 698, 599. HRMS (EI) calcd for $\mathrm{C}_{23} \mathrm{H}_{27} \mathrm{NO}_{3}[\mathrm{M}]^{+} 365.1991$, found 365.1987.

(E)-4-Phenylbut-3-en-1-ol (3I)<smiles>OCC/C=C/c1ccccc1</smiles>

Following general procedure GP2 using (E)-3-phenylprop-2-en-1-ol (40.3 mg, $0.3 \mathrm{mmol}, 1.0$ equiv), diethyl 4-(hydroxymethyl)-2,6-dimethyl-1,4-dihydropyridine-3,5-dicarboxylate (141.0 mg, $0.45 \mathrm{mmol}, 1.5$ equiv), 
DMDC (127 mg, $0.9 \mathrm{mmol}, 3.0$ equiv), (phen) $\mathrm{NiCl}_{2}$ (4.7 mg, $5 \mathrm{~mol} \%$ ), and 4CzIPN (7.1 mg, $3 \mathrm{~mol} \%$ ) in DMF ( $3 \mathrm{~mL}, 0.1 \mathrm{M})$. The reaction was stirred under blue LEDs at $\mathrm{rt}$ for $16 \mathrm{~h}$. Silica gel purification using an automated system (hexanes/EtOAc, 0 to $10 \%)$ gave the product as a colorless oil $(25.3 \mathrm{mg}, 57 \%)$. ${ }^{1} \mathrm{H}$ NMR $\left(500 \mathrm{MHz}, \mathrm{CDCl}_{3}\right) \delta 7.36(\mathrm{~d}, J=7.5 \mathrm{~Hz}, 2 \mathrm{H}), 7.31(\mathrm{dd}, J=8.4,6.9 \mathrm{~Hz}, 2 \mathrm{H}), 7.25-7.19(\mathrm{~m}, 1 \mathrm{H}), 6.51(\mathrm{~d}, J$ $=15.8 \mathrm{~Hz}, 1 \mathrm{H}), 6.21(\mathrm{dt}, J=16.0,7.1 \mathrm{~Hz}, 1 \mathrm{H}), 3.80-3.70(\mathrm{~m}, 2 \mathrm{H}), 2.50(\mathrm{td}, J=6.3,1.5 \mathrm{~Hz}, 2 \mathrm{H}), 1.47(\mathrm{~s}$, 1H). ${ }^{13} \mathrm{C} \mathrm{NMR}\left(126 \mathrm{MHz}, \mathrm{CDCl}_{3}\right) \delta 137.4,133.0,128.7,127.4,126.4,126.2,62.2,36.6$. IR (neat, $\left.\mathrm{cm}^{-1}\right): 3375$, 2923, 2853, 1768, 1727, 1598, 1492, 1450, 1371, 1336, 1312, 1272, 1227, 1197, 1089, 1043, 1029, 966, 747, 726, 700. HRMS (EI) calcd for $\mathrm{C}_{10} \mathrm{H}_{12} \mathrm{O}[\mathrm{M}]^{+} 148.0889$, found 148.0879 .

$(3 \mathrm{a} R, 4 R, 6 R, 6 \mathrm{a} R)$-4-Cinnamyl-6-methoxy-2,2-dimethyltetrahydrofuro[3,4- $d][1,3]$ dioxole $(\mathbf{4 a})$<smiles>COC1OC(C/C=C/c2ccccc2)C(OC(C)(C)C)C1O</smiles>

Following general procedure GP2 using (E)-3-phenylprop-2-en-1-ol (40.3 mg, $0.3 \mathrm{mmol}, 1.0$ equiv), diethyl 4-((3aR,6R,6aR)-6-methoxy-2,2-dimethyltetrahydrofuro[3,4- $d][1,3]$ dioxol-4-yl)-2,6-dimethyl-1,4-

dihydropyridine-3,5-dicarboxylate ( $191.5 \mathrm{mg}, 0.45 \mathrm{mmol}, 1.5$ equiv), DMDC (127 mg, $0.9 \mathrm{mmol}, 3.0$ equiv), (phen) $\mathrm{NiCl}_{2}(4.7 \mathrm{mg}, 5 \mathrm{~mol} \%)$, and 4CzIPN (7.1 mg, $3 \mathrm{~mol} \%$ ) in DMF (3 mL, $\left.0.1 \mathrm{M}\right)$. The reaction was stirred under blue LEDs at $\mathrm{rt}$ for $16 \mathrm{~h}$. Silica gel purification using an automated system (hexanes/EtOAc, 0 to $10 \%)$ gave the product as a colorless oil $(46.2 \mathrm{mg}, 53 \%) .{ }^{1} \mathrm{H}$ NMR $\left(500 \mathrm{MHz}, \mathrm{CDCl}_{3}\right) \delta 7.37(\mathrm{~d}, J=7.2$ $\mathrm{Hz}, 2 \mathrm{H}), 7.31(\mathrm{t}, J=7.6 \mathrm{~Hz}, 2 \mathrm{H}), 7.25-7.20(\mathrm{~m}, 1 \mathrm{H}), 6.49(\mathrm{~d}, J=15.9 \mathrm{~Hz}, 1 \mathrm{H}), 6.22(\mathrm{ddd}, J=15.9,7.7,6.4$ $\mathrm{Hz}, 1 \mathrm{H}), 4.99(\mathrm{~s}, 1 \mathrm{H}), 4.66-4.62(\mathrm{~m}, 2 \mathrm{H}), 4.34(\mathrm{t}, J=7.7 \mathrm{~Hz}, 1 \mathrm{H}), 3.38(\mathrm{~s}, 3 \mathrm{H}), 2.62-2.56(\mathrm{~m}, 1 \mathrm{H}), 2.50-$ $2.44(\mathrm{~m}, 1 \mathrm{H}), 1.50(\mathrm{~s}, 3 \mathrm{H}), 1.33(\mathrm{~s}, 3 \mathrm{H}) .{ }^{13} \mathrm{C} \mathrm{NMR}(126 \mathrm{MHz}, \mathrm{CDCl} 3) \delta 132.5,128.4,127.2,126.0,125.8$, 114.5, 109.5, 107.0, 86.5, 85.5, 83.4, 54.8, 38.6, 26.4, 24.9. IR (neat, $\mathrm{cm}^{-1}$ ): 2989, 2935, 1496, 1449, 1373, $1272,1239,1210,1193,1161,1106,1092,1060,1026,965,871,743,694,514,493$. HRMS (EI) calcd for $\mathrm{C}_{17} \mathrm{H}_{22} \mathrm{O}_{4}[\mathrm{M}]^{+} 290.1518$, found 290.1509 .

(3aR,5aS,8bR)-5-Cinnamyl-2,2,7,7-tetramethyltetrahydro-5H-bis([1,3]dioxolo)[4,5- $b: 4 ', 5$ '- $d]$ pyran (4b)<smiles>CC1(C)O[C@H]2[C@@H]3OC(C)(C)O[C@@H]3[C@@H]3OC(C)(C)O[C@H]3[C@H]2O1</smiles>

Following general procedure GP2 using (E)-3-phenylprop-2-en-1-ol (40.3 mg, $0.3 \mathrm{mmol}, 1.0$ equiv), diethyl 2,6-dimethyl-4-((3aR,5aS,8aS,8bR)-2,2,7,7-tetramethyltetrahydro-5H-bis([1,3]dioxolo)[4,5- $b: 4$ ',5'- $d]$ pyran5-yl)-1,4-dihydropyridine-3,5-dicarboxylate ( $216.7 \mathrm{mg}, 0.45 \mathrm{mmol}, 1.5$ equiv), DMDC ( $0.9 \mathrm{mmol}, 3.0$ equiv), (phen) $\mathrm{NiCl}_{2}$ (4.7 mg, $5 \mathrm{~mol} \%$ ), and 4CzIPN (127 mg, $7.1 \mathrm{mg}, 3 \mathrm{mmol} \%$ ) in DMF (3 mL, $\left.0.1 \mathrm{M}\right)$. The reaction was stirred under blue LEDs at $\mathrm{rt}$ for $16 \mathrm{~h}$. Silica gel purification using an automated system (hexanes/EtOAc, 0 to $10 \%$ ) gave the product as a colorless oil $(54.8 \mathrm{mg}, 53 \%) .{ }^{1} \mathrm{H} \mathrm{NMR}\left(500 \mathrm{MHz}, \mathrm{CDCl}_{3}\right)$ $\delta 7.36(\mathrm{~d}, J=7.5 \mathrm{~Hz}, 2 \mathrm{H}), 7.29(\mathrm{dd}, J=7.5,7.3 \mathrm{~Hz}, 2 \mathrm{H}), 7.20(\mathrm{t}, J=7.3 \mathrm{~Hz}, 1 \mathrm{H}), 6.54(\mathrm{~d}, J=15.9 \mathrm{~Hz}, 1 \mathrm{H})$, $6.25(\mathrm{dt}, J=15.7,7.0 \mathrm{~Hz}, 1 \mathrm{H}), 5.56(\mathrm{~d}, J=5.1 \mathrm{~Hz}, 1 \mathrm{H}), 4.60(\mathrm{dd}, J=7.9,2.3 \mathrm{~Hz}, 1 \mathrm{H}), 4.31(\mathrm{dd}, J=5.1,2.3$ $\mathrm{Hz}, 1 \mathrm{H}), 4.20(\mathrm{dd}, J=8.0,1.9 \mathrm{~Hz}, 1 \mathrm{H}), 3.86(\mathrm{t}, J=6.7 \mathrm{~Hz}, 1 \mathrm{H}), 2.56(\mathrm{~m}, 2 \mathrm{H}), 1.55(\mathrm{~s}, 3 \mathrm{H}), 1.49(\mathrm{~s}, 3 \mathrm{H}), 1.37$ (s, 3H), 1.34 (s, 3H). ${ }^{13} \mathrm{C}$ NMR $\left(126 \mathrm{MHz}, \mathrm{CDCl}_{3}\right) \delta 137.7,132.4,128.6,127.2,126.3,126.1,109.3,108.6$, 96.8, 72.4, 71.0, 70.7, 67.7, 33.9, 26.2, 26.2, 25.1, 24.6. IR (neat, $\left.\mathrm{cm}^{-1}\right): 2981,2919,1381,1372,1255,1210$, $1166,1142,1102,1067,1035,997,966,916,901,887,864,804,743,693$. HRMS (EI) calcd for $\mathrm{C}_{19} \mathrm{H}_{23} \mathrm{O}_{5}$ $\left[\mathrm{M}-\mathrm{CH}_{3}\right]^{+} 331.1545$, found 331.1537 . 


\section{Photoredox/Nickel Dual-Catalyzed Sulfonylation of Allyl Carbonates}

\subsection{General Procedure (GP3)}

To an $8-\mathrm{mL}$ reaction vial equipped with a stir bar and septa screw cap were added sodium benzenesulfinates ( $0.6 \mathrm{mmol}, 2.0$ equiv), allylic carbonates ( $0.3 \mathrm{mmol}, 1.0$ equiv), (phen) $\mathrm{NiCl}_{2}(4.7 \mathrm{mg}, 5 \mathrm{~mol} \%)$, and $4 \mathrm{CzIPN}$ ( $7.1 \mathrm{mg}, 3 \mathrm{~mol} \%$ ). The reaction vial was subsequently closed, and three vacuum/argon cycles were performed, followed by addition of $3 \mathrm{~mL}$ of dry DMF $(0.1 \mathrm{M})$. After stirring under blue LEDs for $16 \mathrm{~h}$, the reaction was diluted with $30 \mathrm{~mL}$ of EtOAc and washed three times with $10 \mathrm{~mL}$ of saturated $\mathrm{Na}_{2} \mathrm{CO}_{3}$ and subsequently three times with $10 \mathrm{~mL}$ of brine. The organic phase was dried $\left(\mathrm{MgSO}_{4}\right)$, and the solvent was removed by rotovap. The final product was purified by flash column chromatography, using hexanes and EtOAc as eluent.

\subsection{Characterization Data}

(Cinnamylsulfonyl)benzene (5a)<smiles>O=S(=O)(C/C=C/c1ccccc1)c1ccccc1</smiles>

Following general procedure GP3 using cinnamyl methyl carbonate $(57.7 \mathrm{mg}, 0.3 \mathrm{mmol}, 1.0$ equiv), sodium benzenesulfinate ( $98.5 \mathrm{mg}, 0.6 \mathrm{mmol}, 2.0$ equiv), (phen) $\mathrm{NiCl}_{2}(4.7 \mathrm{mg}, 5 \mathrm{~mol} \%$ ), and 4CzIPN (7.1 mg, 3 $\mathrm{mol} \%$ ) in DMF ( $3 \mathrm{~mL}, 0.1 \mathrm{M})$. The reaction was stirred under blue LEDs at $\mathrm{rt}$ for $16 \mathrm{~h}$. Silica gel purification using an automated system (hexanes/EtOAc, 10 to 30\%) gave the product as a white solid (57.6 mg, 74\%). $\mathrm{mp}=67-72{ }^{\circ} \mathrm{C} .{ }^{1} \mathrm{H}$ NMR $\left(500 \mathrm{MHz}, \mathrm{CDCl}_{3}\right) \delta 7.88(\mathrm{ddd}, J=8.4,8.4,1.1 \mathrm{~Hz}, 2 \mathrm{H}), 7.67-7.58(\mathrm{~m}, 1 \mathrm{H})$, $7.54(\mathrm{t}, J=7.7 \mathrm{~Hz}, 2 \mathrm{H}), 7.34-7.28(\mathrm{~m}, 5 \mathrm{H}), 6.37(\mathrm{~d}, J=15.8 \mathrm{~Hz}, 1 \mathrm{H}), 6.10(\mathrm{dt}, J=15.6,7.6 \mathrm{~Hz}, 1 \mathrm{H}), 3.95$ $(\mathrm{ddd}, J=7.6,7.6,1.1 \mathrm{~Hz}, 2 \mathrm{H}) .{ }^{13} \mathrm{C} \mathrm{NMR}\left(126 \mathrm{MHz}, \mathrm{CDCl}_{3}\right) \delta 139.1,138.2,135.7,133.7,129.0,128.6(2 \mathrm{C})$, 128.4,126.5, 115.0, 60.4. IR (neat, $\mathrm{cm}^{-1}$ ): 2975, 2903, 1317, 1306, 1295, 1234, 1149, 1084, 1025, 967, 903, 770, 751, 688, 619, 595, 564, 530, 495. HRMS (EI) calcd for $\mathrm{C}_{15} \mathrm{H}_{14} \mathrm{O}_{2} \mathrm{~S}[\mathrm{M}]^{+}$258.0715, found 258.0722.

1-(Cinnamylsulfonyl)-4-methylbenzene (5b)<smiles>Cc1ccc(S(=O)(=O)C/C=C/c2ccccc2)cc1</smiles>

Following general procedure GP3 using cinnamyl methyl carbonate $(57.7 \mathrm{mg}, 0.3 \mathrm{mmol}, 1.0$ equiv), sodium 4-methylbenzenesulfinate (106.9 mg, $0.6 \mathrm{mmol}, 2.0$ equiv), (phen) $\mathrm{NiCl}_{2}$ (4.7 mg, $5 \mathrm{~mol} \%$ ), and 4CzIPN (7.1 $\mathrm{mg}, 3 \mathrm{~mol} \%$ ) in DMF ( $3 \mathrm{~mL}, 0.1 \mathrm{M}$ ). The reaction was stirred under blue LEDs at $\mathrm{rt}$ for $16 \mathrm{~h}$. Silica gel purification using an automated system (hexanes/EtOAc, 10 to 30\%) gave the product as a white solid (67.2 $\mathrm{mg}, 82 \%) . \mathrm{mp}=113-116^{\circ} \mathrm{C} .{ }^{1} \mathrm{H} \mathrm{NMR}\left(500 \mathrm{MHz}, \mathrm{CDCl}_{3}\right) \delta 7.82-7.66(\mathrm{~m}, 2 \mathrm{H}), 7.38-7.28(\mathrm{~m}, 7 \mathrm{H}), 6.40$ $(\mathrm{d}, J=15.8 \mathrm{~Hz}, 1 \mathrm{H}), 6.11(\mathrm{dt}, J=15.5,7.5 \mathrm{~Hz}, 1 \mathrm{H}), 3.94(\mathrm{~d}, J=7.7 \mathrm{~Hz}, 2 \mathrm{H}), 2.45(\mathrm{~s}, 3 \mathrm{H}) .{ }^{13} \mathrm{C}$ NMR $(126$ $\mathrm{MHz}, \mathrm{CDCl}_{3}$ ) $\delta 144.6,138.9,135.7,135.5,129.6,128.6,128.4,128.4,126.5,115.3,60.5,21.5$. IR (neat, $\mathrm{cm}^{-}$ $\left.{ }^{1}\right)$ : 3025, 2925, 1479, 1458, 1447, 1397, 1225, 1205, 1135, 1084, 967, 819, 749, 727, 616, 599, 557, 530, 478. HRMS (EI) calcd for $\mathrm{C}_{16} \mathrm{H}_{16} \mathrm{O}_{2} \mathrm{~S}[\mathrm{M}]^{+} 272.0871$, found 272.0869 . 


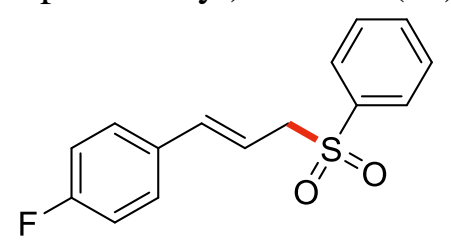

Following general procedure GP3 using (E)-3-(4-fluorophenyl)allyl methyl carbonate $(63.1 \mathrm{mg}, 0.3 \mathrm{mmol}$, 1.0 equiv), sodium benzenesulfinate ( $98.5 \mathrm{mg}, 0.6 \mathrm{mmol}, 2.0$ equiv), (phen) $\mathrm{NiCl}_{2}(4.7 \mathrm{mg}, 5 \mathrm{~mol} \%$ ), and 4CzIPN (7.1 mg, $3 \mathrm{~mol} \%$ ) in DMF ( $3 \mathrm{~mL}, 0.1 \mathrm{M}$ ). The reaction was stirred under blue LEDs at rt for $16 \mathrm{~h}$. Silica gel purification using an automated system (hexanes/EtOAc, 10 to 30\%) gave the product as a white solid (43.0 mg, 52\%). $\mathrm{mp}=89-92{ }^{\circ} \mathrm{C} .{ }^{1} \mathrm{H}$ NMR $\left(500 \mathrm{MHz}, \mathrm{CDCl}_{3}\right) \delta 7.89(\mathrm{dd}, J=8.3,1.3 \mathrm{~Hz}, 2 \mathrm{H}), 7.69-$ $7.64(\mathrm{~m}, 1 \mathrm{H}), 7.56(\mathrm{dd}, J=7.7,7.7 \mathrm{~Hz}, 2 \mathrm{H}), 7.29-7.24(\mathrm{~m}, 2 \mathrm{H}), 7.01(\mathrm{dd}, J=8.7,8.7 \mathrm{~Hz}, 2 \mathrm{H}), 6.36(\mathrm{~d}, J=$ $15.8 \mathrm{~Hz}, 1 \mathrm{H}), 6.03(\mathrm{dt}, J=15.5,7.6 \mathrm{~Hz}, 1 \mathrm{H}), 3.95(\mathrm{dd}, J=7.5,1.1 \mathrm{~Hz}, 2 \mathrm{H}) .{ }^{13} \mathrm{C}$ NMR $\left(126 \mathrm{MHz}, \mathrm{CDCl}_{3}\right) \delta$ 162.0, 138.6, 138.2, 134.0, 132.1, 129.3, 128.6, $128.4(\mathrm{~d}, J=8.2 \mathrm{~Hz}), 115.8(\mathrm{~d}, J=21.7 \mathrm{~Hz}), 115.0(\mathrm{~d}, J=$ $2.3 \mathrm{~Hz}), 60.6 .{ }^{19} \mathrm{~F}\left(471 \mathrm{MHz}, \mathrm{CDCl}_{3}\right) \delta 112.7$. IR (neat, $\left.\mathrm{cm}^{-1}\right): 3082,2963,1508,1447,1306,1226,1148$, $1085,968,904,842,821,765,708,688,601,577,556,528,509$. HRMS (EI) calcd for $\mathrm{C}_{15} \mathrm{H}_{13} \mathrm{FO}_{2} \mathrm{~S}[\mathrm{M}]^{+}$ 276.0620 , found 276.0621 .

(E)-2-(3-(Phenylsulfonyl)prop-1-en-1-yl)naphthalene (5d)<smiles>O=S(=O)(C/C=C/c1ccc2ccccc2c1)c1ccccc1</smiles>

Following general procedure GP3 using (E)-methyl (3-(naphthalen-2-yl)allyl) carbonate (72.7 mg, $0.3 \mathrm{mmol}$, 1.0 equiv), sodium benzenesulfinate $\left(98.5 \mathrm{mg}, 0.6 \mathrm{mmol}, 2.0\right.$ equiv), (phen) $\mathrm{NiCl}_{2}(4.7 \mathrm{mg}, 5 \mathrm{~mol} \%$ ), and 4CzIPN (7.1 mg, $3 \mathrm{~mol} \%$ ) in DMF (3 mL, 0.1 M). The reaction was stirred under blue LEDs at $\mathrm{rt}$ for $16 \mathrm{~h}$. Silica gel purification using an automated system (hexanes/EtOAc, 10 to 30\%) gave the product as a white solid $(43.5 \mathrm{mg}, 47 \%) . \mathrm{mp}=96-99^{\circ} \mathrm{C} .{ }^{1} \mathrm{H}$ NMR $\left(500 \mathrm{MHz}, \mathrm{CDCl}_{3}\right) \delta 7.90(\mathrm{~d}, J=7.9 \mathrm{~Hz}, 2 \mathrm{H}), 7.82-7.75$ (m, 3H), $7.67-7.61(\mathrm{~m}, 2 \mathrm{H}), 7.56-7.51(\mathrm{~m}, 2 \mathrm{H}), 7.50(\mathrm{dd}, J=8.6,1.6 \mathrm{~Hz}, 1 \mathrm{H}), 7.48-7.44(\mathrm{~m}, 2 \mathrm{H}), 6.52$ $(\mathrm{d}, J=15.8 \mathrm{~Hz}, 1 \mathrm{H}), 6.23(\mathrm{dt}, J=15.5,7.6 \mathrm{~Hz}, 1 \mathrm{H}), 4.01(\mathrm{~d}, J=7.6 \mathrm{~Hz}, 2 \mathrm{H}) .{ }^{13} \mathrm{C}$ NMR $\left(126 \mathrm{MHz}, \mathrm{CDCl}_{3}\right)$ $\delta 139.4$ (2C), 138.6, 133.9, 133.5, 133.3, 129.2, 128.7, 128.6, 128.2, 127.8, 127.2, 126.6, 126.6, 123.4, 115.5, 60.8. IR (neat, $\mathrm{cm}^{-1}$ ): 3051, 2962, 1446, 1307, 1291, 1150, 1085, 964, 909, 861, 813, 774, 749, 704, 687, 598, 568, 556, 527, 475. HRMS (EI) calcd for $\mathrm{C}_{19} \mathrm{H}_{16} \mathrm{O}_{2} \mathrm{~S}[\mathrm{M}]^{+}$308.0871, found 308.0867.

(E)-1-Methoxy-4-(3-(phenylsulfonyl)prop-1-en-1-yl)benzene (5e)<smiles>COc1ccc(/C=C/CS(=O)(=O)c2ccccc2)cc1</smiles>

Following general procedure GP3 using (E)-3-(4-methoxyphenyl)allyl methyl carbonate (66.7 mg, $0.3 \mathrm{mmol}$, 1.0 equiv), sodium benzenesulfinate $\left(98.5 \mathrm{mg}, 0.6 \mathrm{mmol}, 2.0\right.$ equiv), (phen) $\mathrm{NiCl}_{2}(4.7 \mathrm{mg}, 5 \mathrm{~mol} \%$ ), and 4CzIPN (7.1 mg, $3 \mathrm{~mol} \%$ ) in DMF (3 mL, $0.1 \mathrm{M}$ ). The reaction was stirred under blue LEDs at $\mathrm{rt}$ for $16 \mathrm{~h}$. Silica gel purification using an automated system (hexanes/EtOAc, 10 to 30\%) gave the product as a white solid (59.6 mg, 69\%). $\mathrm{mp}>230{ }^{\circ} \mathrm{C}$ (decomp.). ${ }^{1} \mathrm{H} \mathrm{NMR}\left(500 \mathrm{MHz}, \mathrm{CDCl}_{3}\right) \delta 7.88(\mathrm{dd}, J=8.2,1.3 \mathrm{~Hz}, 2 \mathrm{H})$, $7.66-7.61(\mathrm{~m}, 1 \mathrm{H}), 7.53(\mathrm{dd}, J=7.8,7.8 \mathrm{~Hz}, 2 \mathrm{H}), 7.24-7.20(\mathrm{~m}, 2 \mathrm{H}), 6.84(\mathrm{dd}, J=9.1,2.5 \mathrm{~Hz}, 2 \mathrm{H}), 6.30$ $(\mathrm{d}, J=15.9 \mathrm{~Hz}, 1 \mathrm{H}), 5.95(\mathrm{dt}, J=15.7,7.7 \mathrm{~Hz}, 1 \mathrm{H}), 3.93(\mathrm{dd}, J=7.8,1.1 \mathrm{~Hz}, 2 \mathrm{H}), 3.81(\mathrm{~s}, 3 \mathrm{H}) .{ }^{13} \mathrm{C} \mathrm{NMR}$ $\left(126 \mathrm{MHz}, \mathrm{CDCl}_{3}\right) \delta 160.0,138.8,138.6,133.8,129.2,129.2,128.7,128.1,114.2,112.7,60.8,55.4$. IR (neat, 
$\left.\mathrm{cm}^{-1}\right): 3003,2981,1709,1607,1511,1458,1446,1304,1223,1176,1146,1085,1030,829,749,726,688$, 601, 561, 529. HRMS (EI) calcd for $\mathrm{C}_{16} \mathrm{H}_{16} \mathrm{O}_{3} \mathrm{~S}[\mathrm{M}]^{+}$288.0820, found 288.0817.

(E)-((5,9-Dimethyldeca-2,8-dien-1-yl)sulfonyl)benzene (5f)<smiles>CC(C)=CCCC(C)CC=CCS(=O)(=O)c1ccccc1</smiles>

Following general procedure GP3 using (E)-5,9-dimethyldeca-2,8-dien-1-yl methyl carbonate (72.1 mg, 0.3 mmol, 1.0 equiv), sodium benzenesulfinate ( $98.5 \mathrm{mg}, 0.6 \mathrm{mmol}, 2.0$ equiv), (phen) $\mathrm{NiCl}_{2}$ ( $4.7 \mathrm{mg}, 5 \mathrm{~mol} \%$ ), and 4CzIPN (7.1 mg, $3 \mathrm{~mol} \%$ ) in DMF ( $3 \mathrm{~mL}, 0.1 \mathrm{M})$. The reaction was stirred under blue LEDs at $\mathrm{rt}$ for 16 h. Silica gel purification using an automated system (hexanes/EtOAc, 10 to 30\%) gave the product as a colorless, sticky oil (51.5 mg, 56\%). ${ }^{1} \mathrm{H}$ NMR $\left(500 \mathrm{MHz}, \mathrm{CDCl}_{3}\right) \delta$ 7.90-7.86 (m, 2H), $7.66-7.60(\mathrm{~m}, 1 \mathrm{H})$, $7.55(\mathrm{dd}, J=7.7,7.7 \mathrm{~Hz}, 2 \mathrm{H}), 5.55-5.50(\mathrm{~m}, 1 \mathrm{H}), 5.42-5.36(\mathrm{~m}, 1 \mathrm{H}), 5.07-5.03(\mathrm{~m}, 1 \mathrm{H}), 3.77(\mathrm{~d}, J=7.5$ $\mathrm{Hz}, 2 \mathrm{H}), 2.05-2.00(\mathrm{~m}, 1 \mathrm{H}), 1.97-1.81(\mathrm{~m}, 3 \mathrm{H}), 1.69(\mathrm{~s}, 3 \mathrm{H}), 1.60(\mathrm{~s}, 3 \mathrm{H}), 1.44-1.38(\mathrm{~m}, 1 \mathrm{H}), 1.25-1.19$ $(\mathrm{m}, 1 \mathrm{H}), 1.10-1.03(\mathrm{~m}, 1 \mathrm{H}), 0.76(\mathrm{~d}, J=6.7 \mathrm{~Hz}, 3 \mathrm{H}) .{ }^{13} \mathrm{C} \mathrm{NMR}\left(126 \mathrm{MHz}, \mathrm{CDCl}_{3}\right) \delta 140.3,138.4,133.4$, 131.2, 128.9, 128.4, 124.5, 116.9, 60.1, 39.8, 36.4, 32.2, 25.6, 25.4, 19.1, 17.5. IR (neat, $\mathrm{cm}^{-1}$ ): 2961, 2914, 1745, 1586, 1447, 137, 1319, 1307, 1237, 1144, 1086, 1025, 999, 971, 793, 731, 689, 600, 555, 530. HRMS (EI) calcd for $\mathrm{C}_{18} \mathrm{H}_{26} \mathrm{O}_{2} \mathrm{~S}[\mathrm{M}]^{+}$306.1654, found 306.1650. 


\section{Spectral Data}

${ }^{1} \mathrm{H}-\mathrm{NMR}$ and ${ }^{13} \mathrm{C}-\mathrm{NMR}$ of $(E)$-(3-Cyclohexylprop-1-en-1-yl)benzene (2a \& 3a)<smiles>C(=C/c1ccccc1)\CC1CCCCC1</smiles>

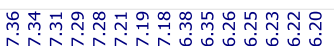

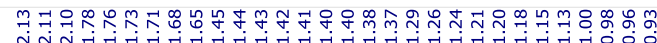

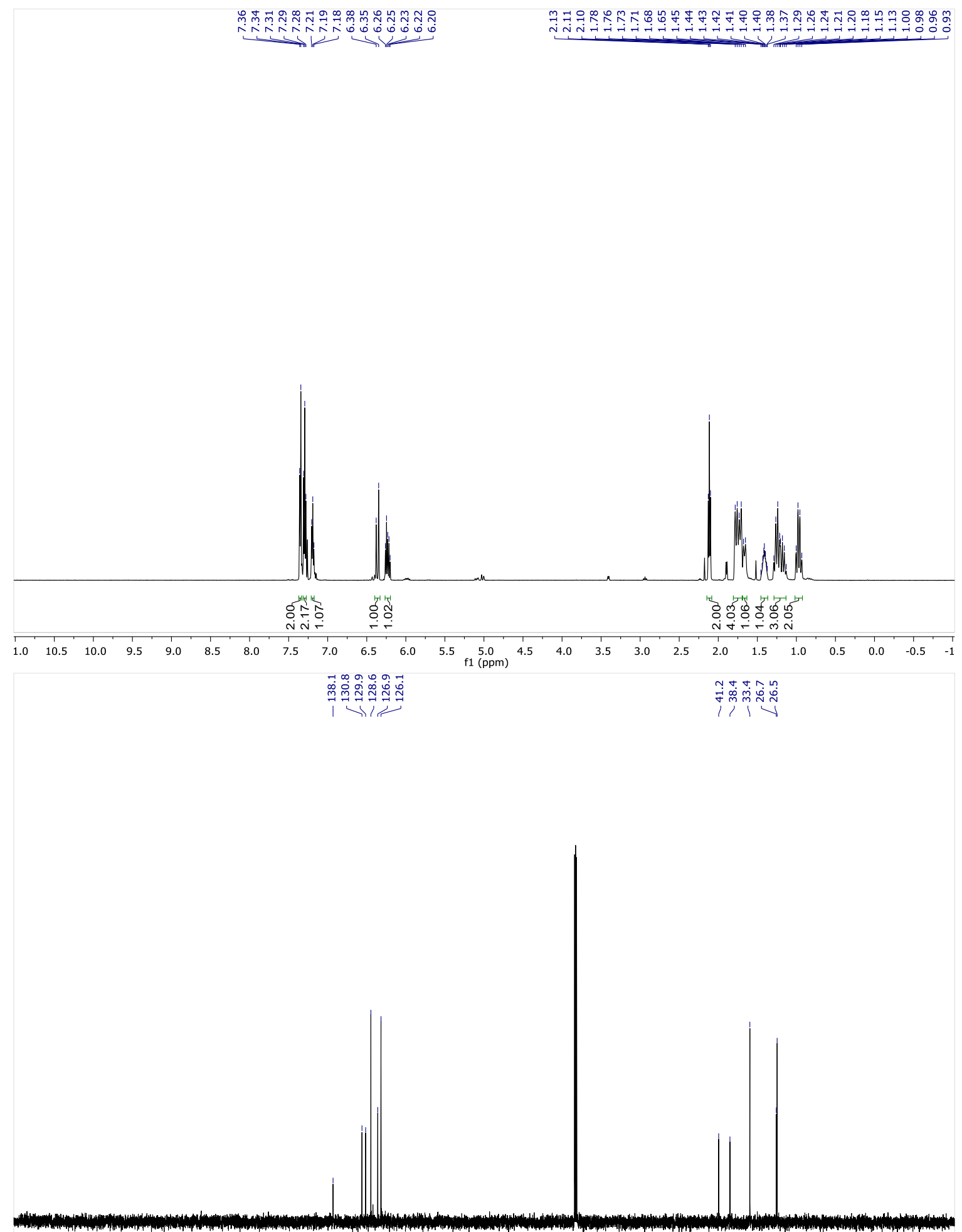

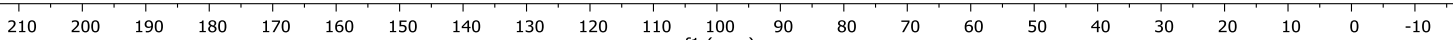


${ }^{1} \mathrm{H}-\mathrm{NMR}$ and ${ }^{13} \mathrm{C}-\mathrm{NMR}$ of $(E)$-(4-Methylpent-1-en-1-yl)benzene (2b \& $\left.\mathbf{3 b}\right)$<smiles>CC(C)CC=Cc1ccccc1</smiles>

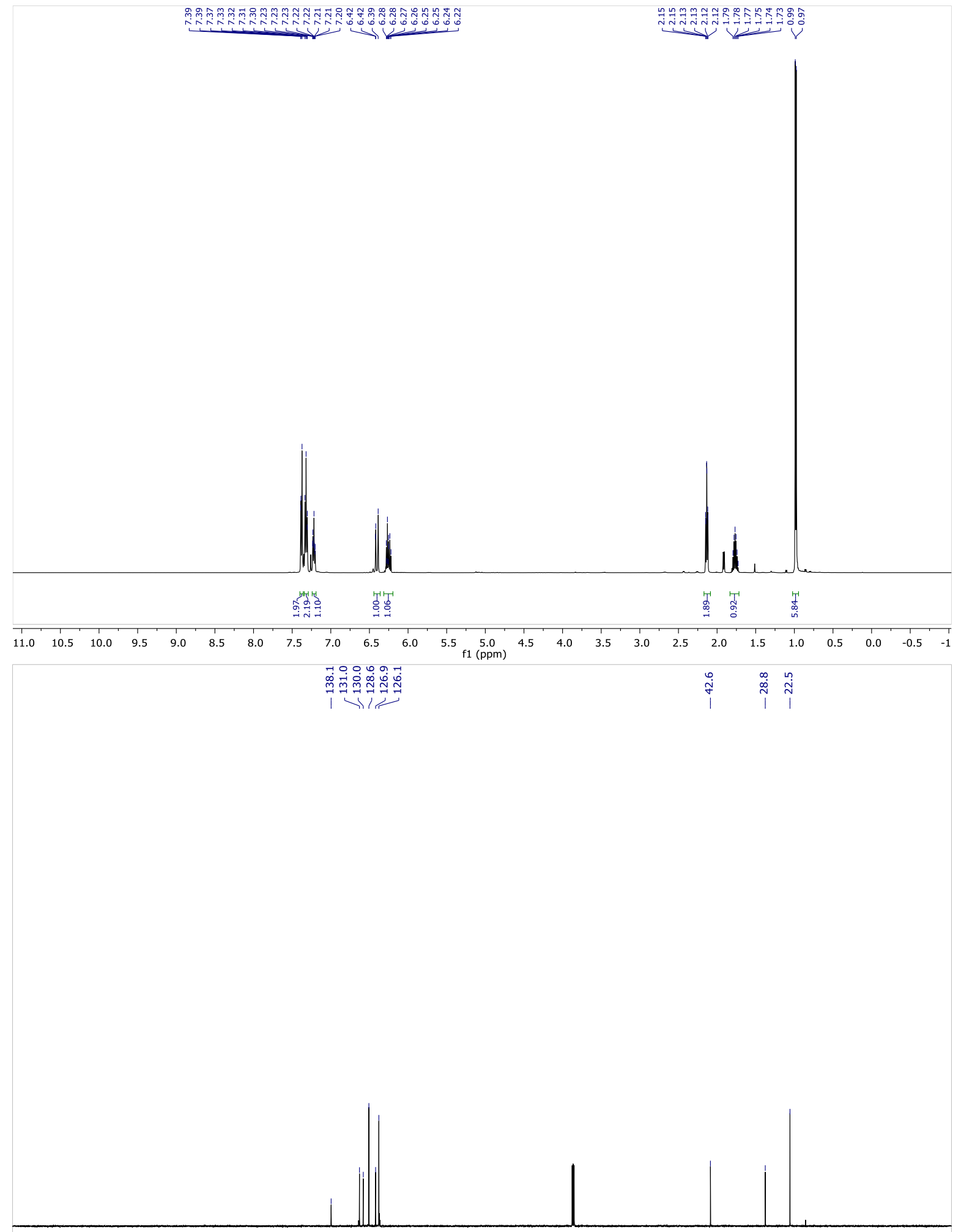

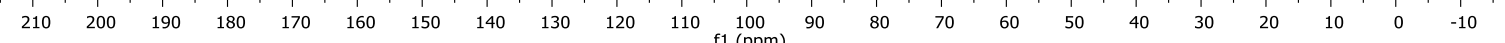


${ }^{1} \mathrm{H}-\mathrm{NMR}$ and ${ }^{13} \mathrm{C}-\mathrm{NMR}$ of $(E)$-2-(4-Methylpent-1-en-1-yl)naphthalene (2c)<smiles>CC(C)C/C=C/c1ccc2ccccc2c1</smiles>

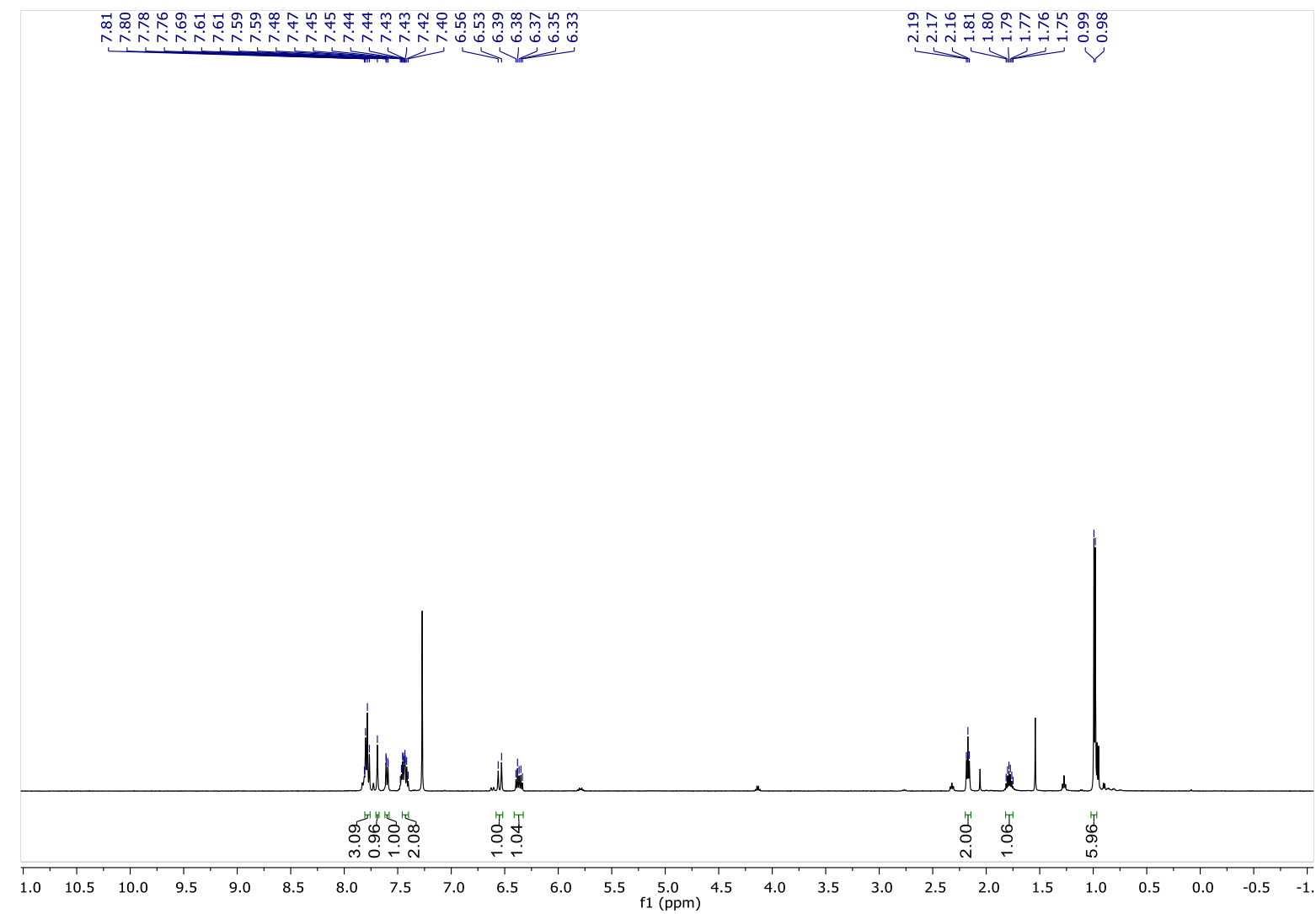

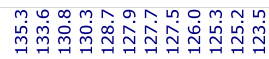

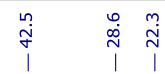

$\begin{array}{lllllllllllllllllllllll}210 & 200 & 190 & 180 & 170 & 160 & 150 & 140 & 130 & 120 & 110 & 100 & 90 & 80 & 70 & 60 & 50 & 40 & 30 & 20 & 10 & 0 & -10\end{array}$ 
${ }^{1} \mathrm{H}-\mathrm{NMR},{ }^{13} \mathrm{C}-\mathrm{NMR}$ and ${ }^{19}$ F-NMR of (E)-1-Fluoro-4-(4-methylpent-1-en-1-yl)benzene (2d)
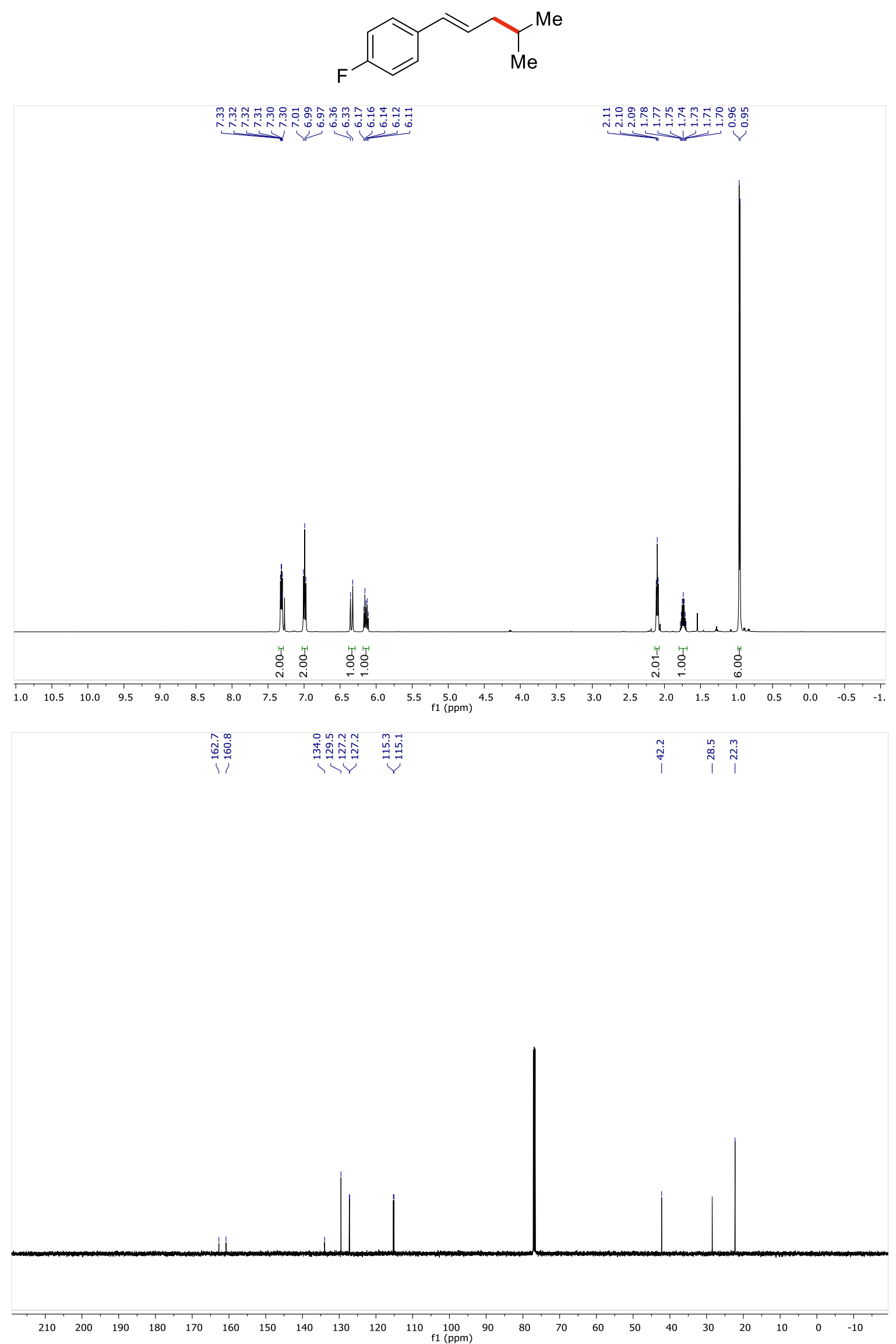


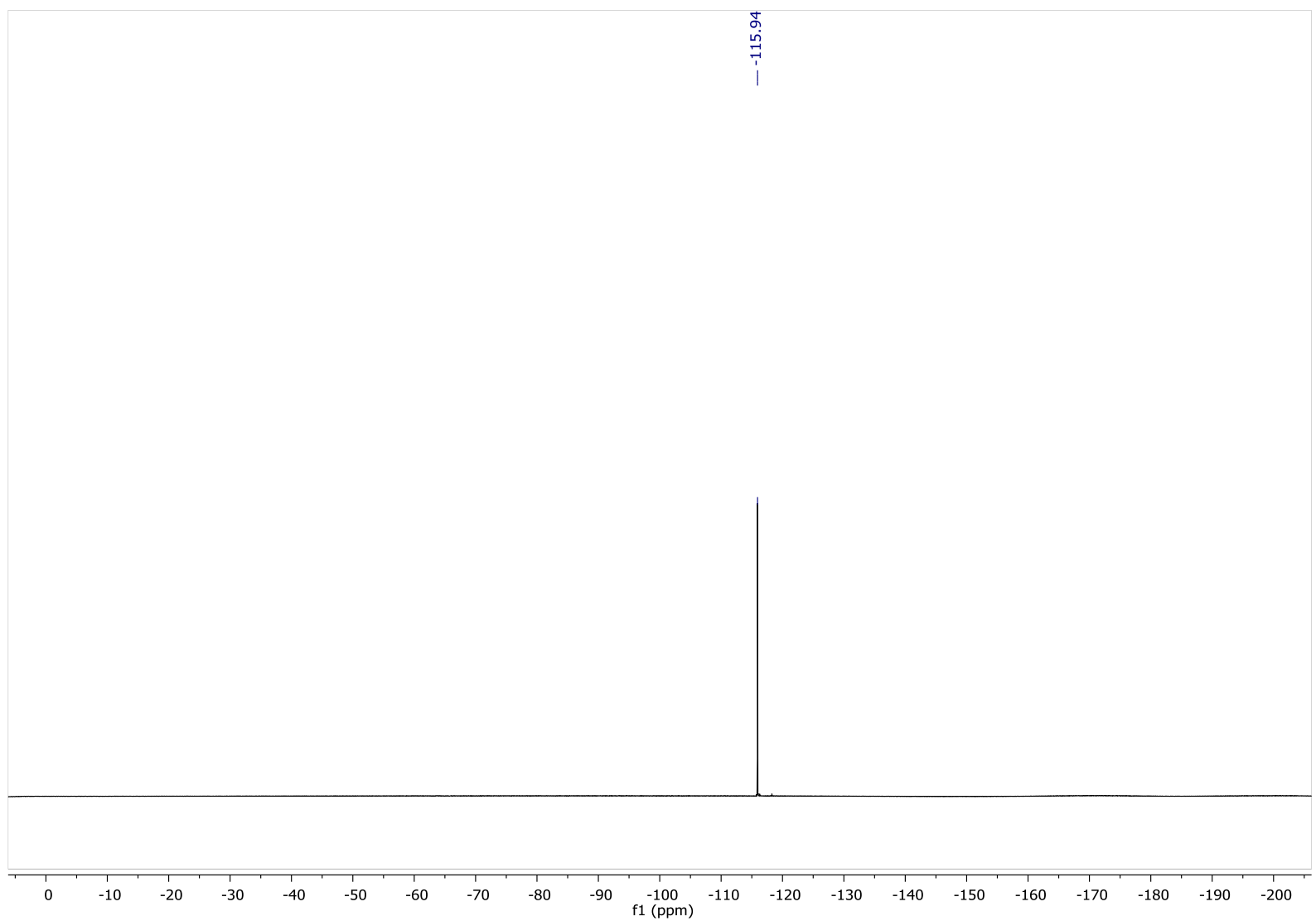


${ }^{1} \mathrm{H}-\mathrm{NMR}$ and ${ }^{13} \mathrm{C}-\mathrm{NMR}$ of $(E)-1,2,3-$ Trimethoxy-5-(4-methylpent-1-en-1-yl)benzene (2e)<smiles>COc1cc(/C=C/CC(C)C)cc(OC)c1OC</smiles>

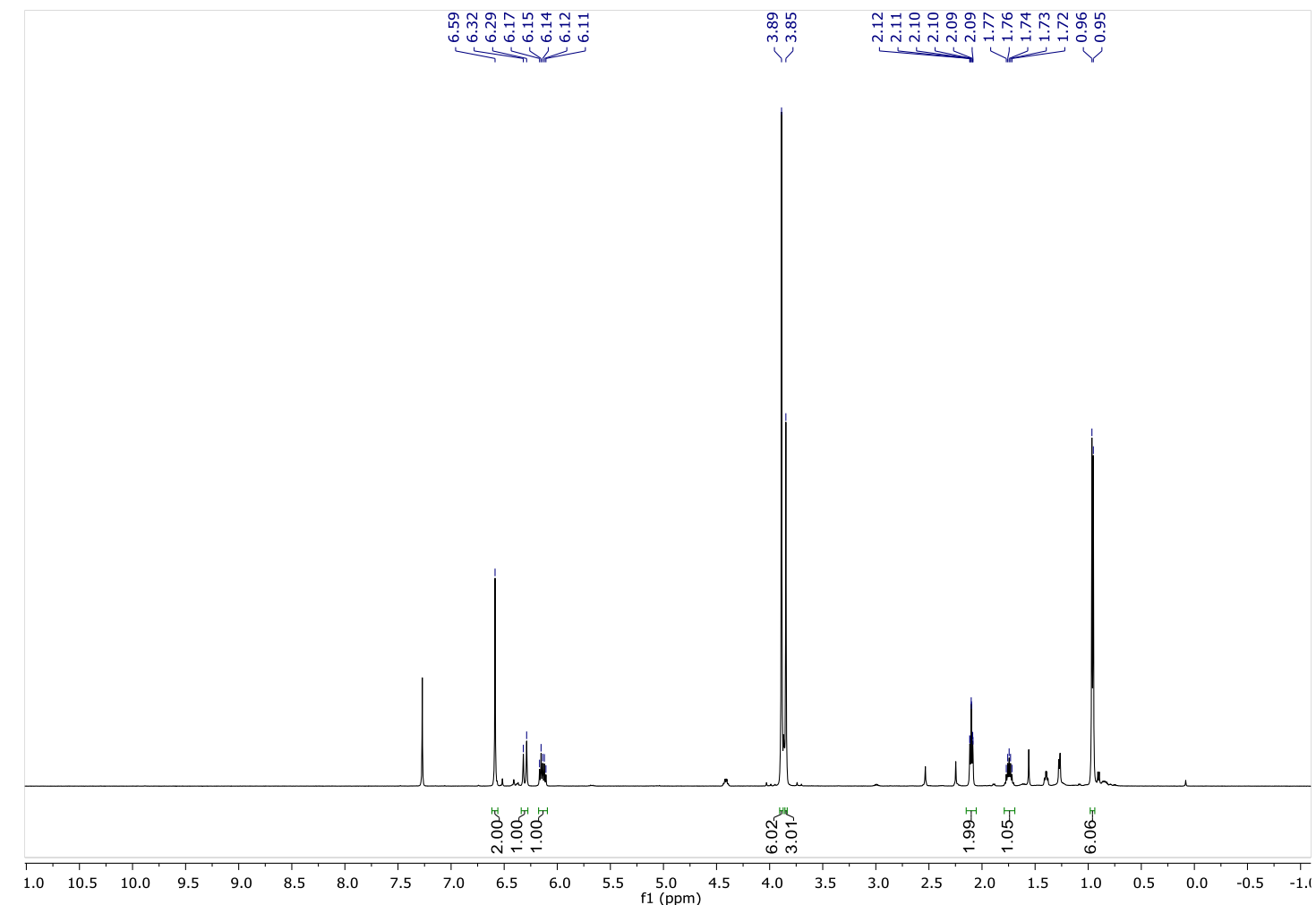

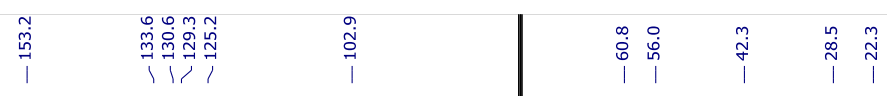

$\begin{array}{llllllllllllllllllllllllllllllllll}210 & 200 & 190 & 180 & 170 & 160 & 150 & 140 & 130 & 120 & 110 & 100 & 90 & 80 & 70 & 60 & 50 & 40 & 30 & 20 & 10 & 0 & -10\end{array}$ 
${ }^{1} \mathrm{H}-\mathrm{NMR}$ and ${ }^{13} \mathrm{C}-\mathrm{NMR}$ of (E)-(6,10-Dimethylundeca-4,9-dien-2-yl)benzene (2f)<smiles>CC(C)=CCCC(C)C=CCC(C)c1ccccc1</smiles>

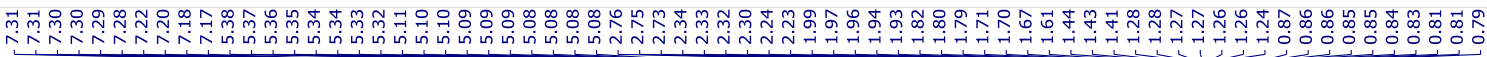
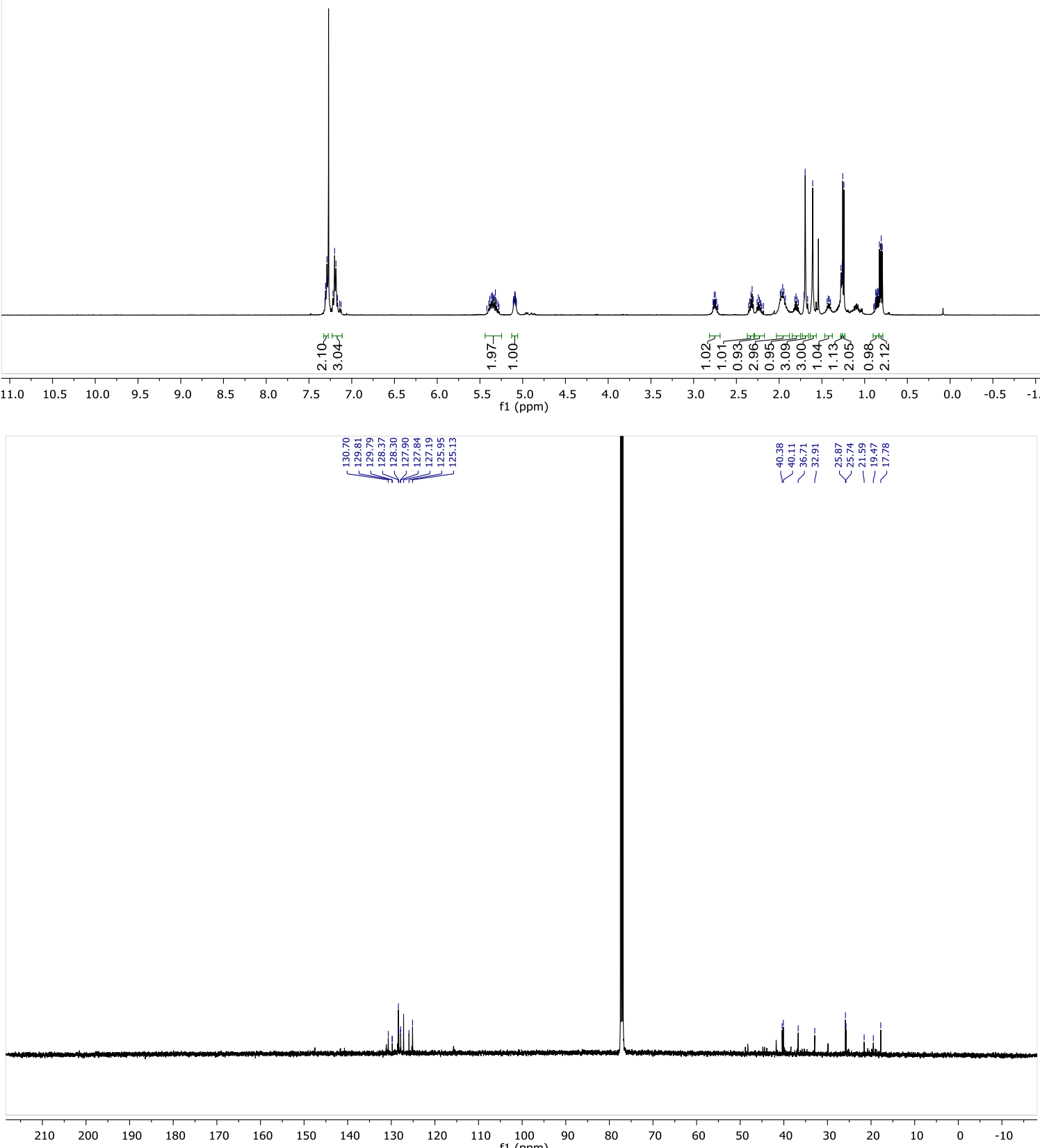
${ }^{1} \mathrm{H}-\mathrm{NMR}$ and ${ }^{13} \mathrm{C}-\mathrm{NMR}$ of $(E)$-(4,9-Dimethyldeca-1,8-dien-1-yl)benzene (2g)<smiles>CC(C)=CCCC(C)CC=Cc1ccccc1</smiles>

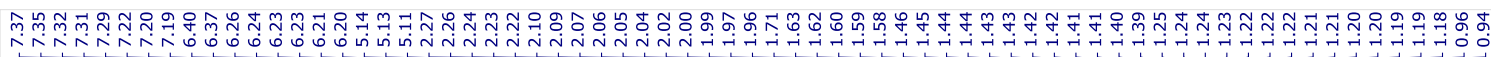

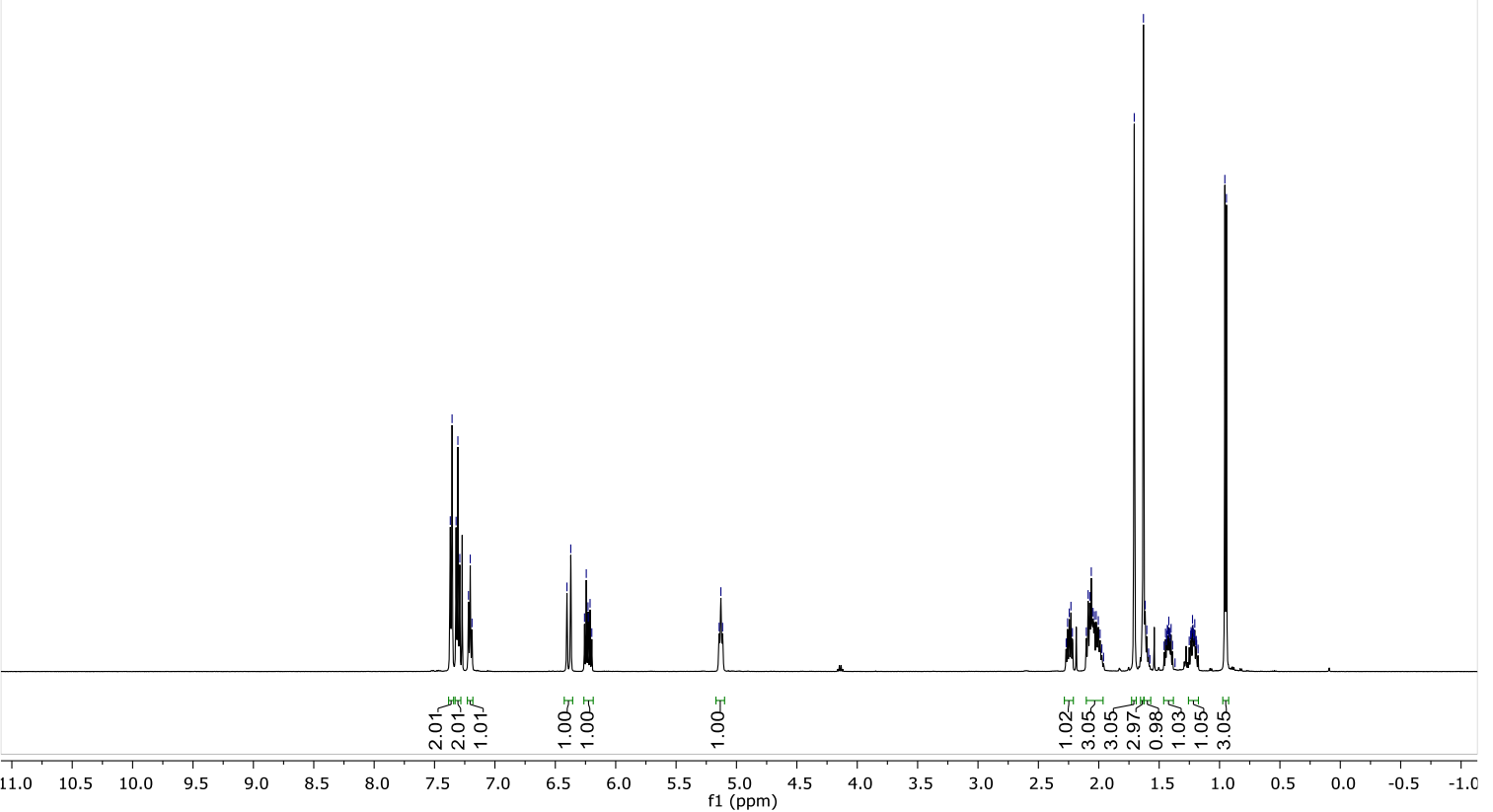

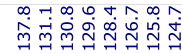

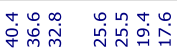

1 긱

$111 \vee 1$

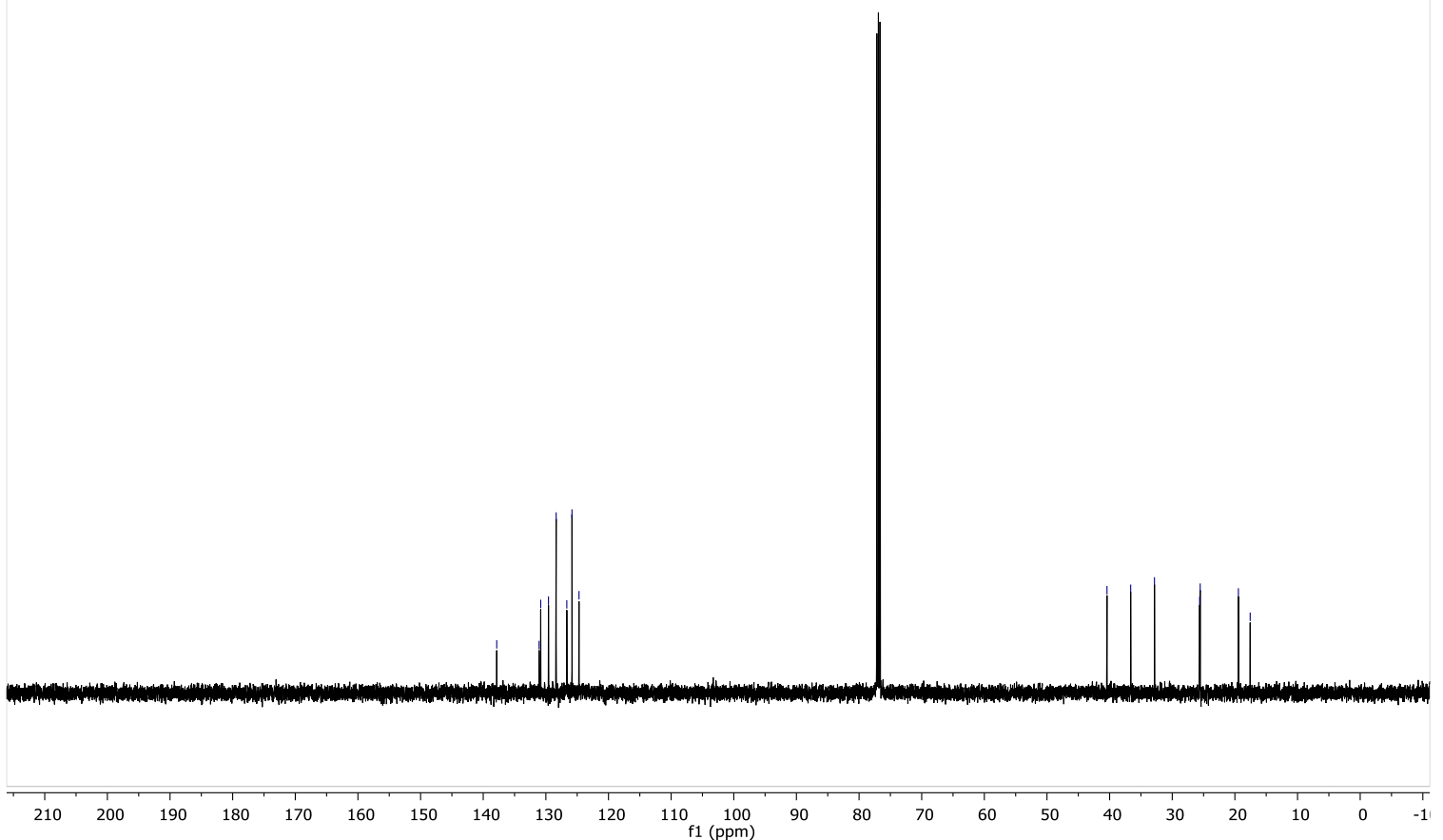


${ }^{1} \mathrm{H}-\mathrm{NMR}$ and ${ }^{13} \mathrm{C}-\mathrm{NMR}$ of Cinnamylcycloheptane (2h)
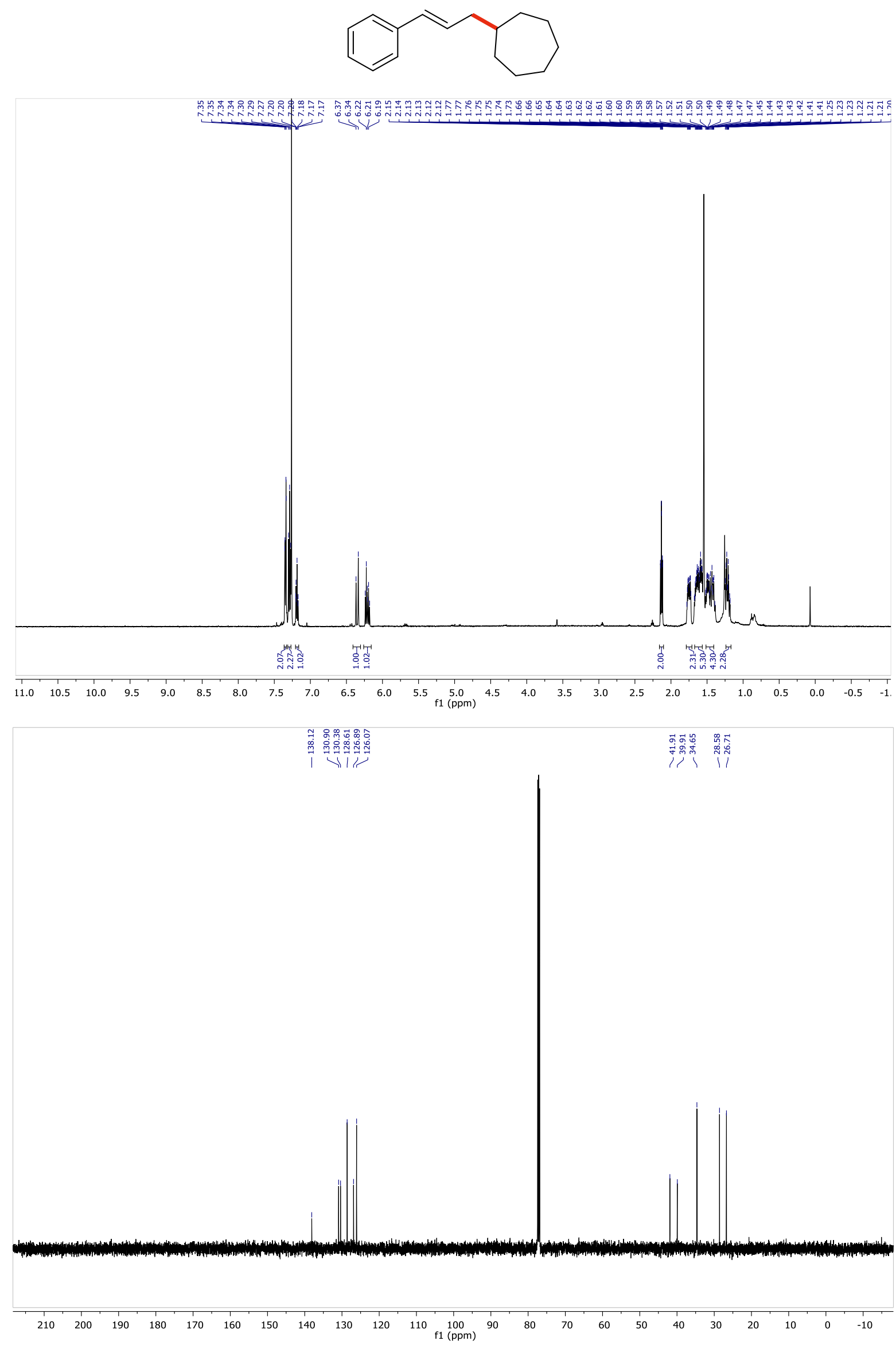
${ }^{1} \mathrm{H}-\mathrm{NMR}$ and ${ }^{13} \mathrm{C}-\mathrm{NMR}$ of $(E)$-(4-(Benzyloxy)but-1-en-1-yl)benzene (2i)<smiles>C(=C/c1ccccc1)\CCOCc1ccccc1</smiles>
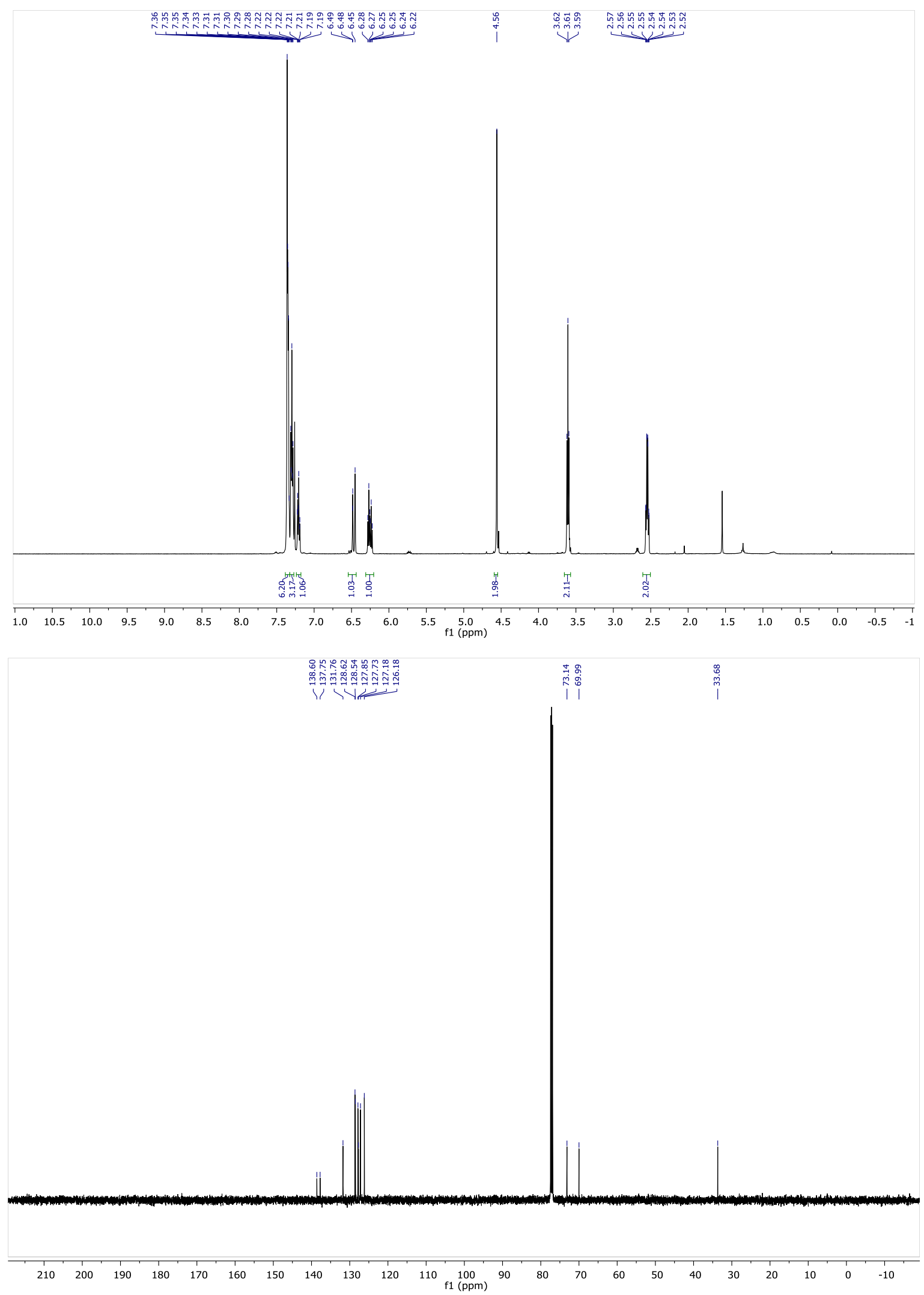
${ }^{1} \mathrm{H}$-NMR and ${ }^{13} \mathrm{C}-\mathrm{NMR}$ of (E)-2-Cinnamylbicyclo[2.2.1]heptane (2j)

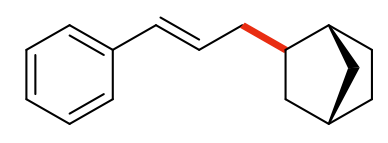

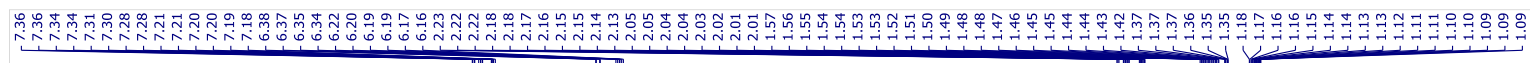
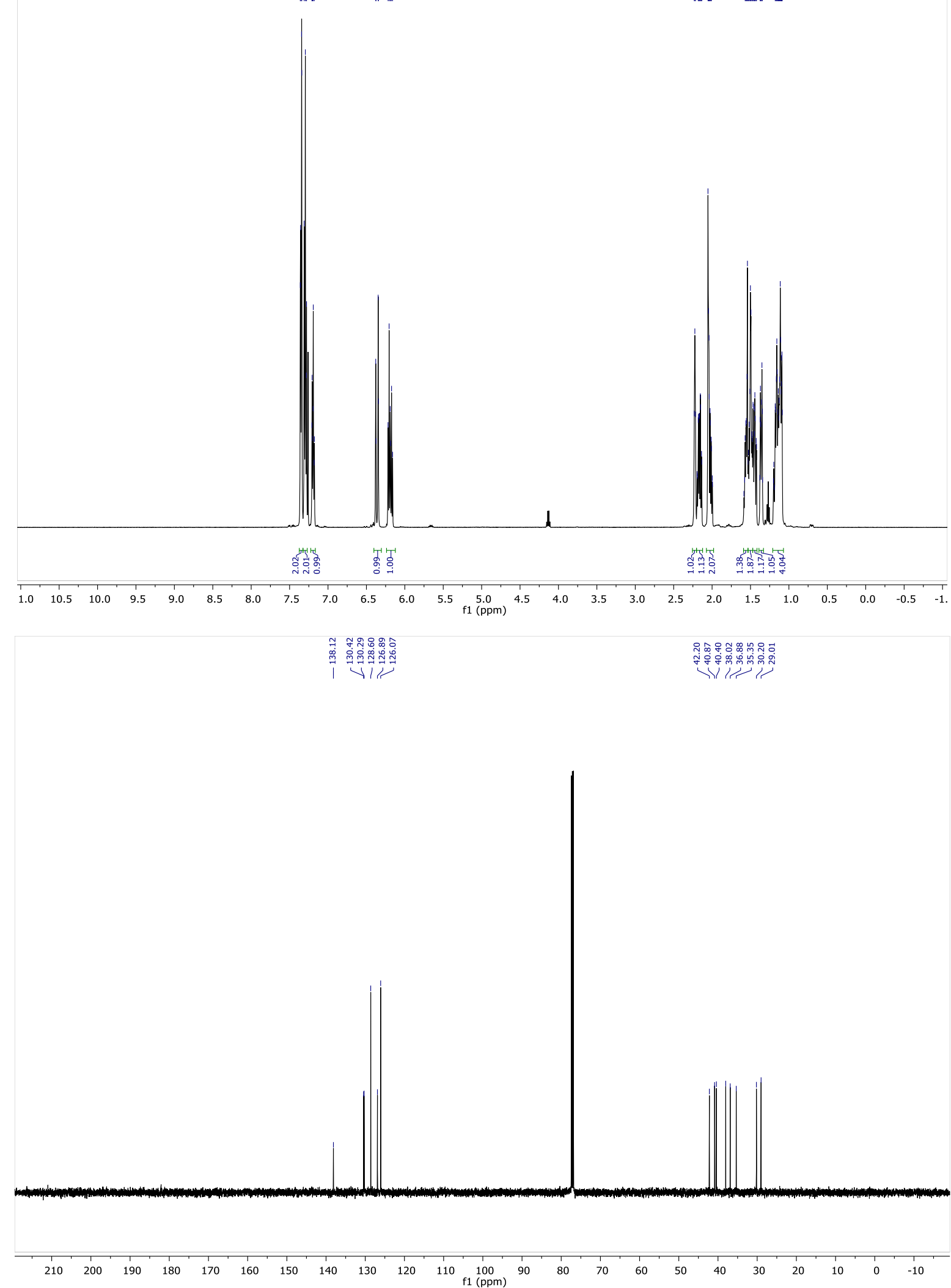
${ }^{1} \mathrm{H}-\mathrm{NMR},{ }^{13} \mathrm{C}-\mathrm{NMR}$ and ${ }^{19} \mathrm{~F}-\mathrm{NMR}$ of (E)-1-(3-Cyclohexylprop-1-en-1-yl)-3,5-difluorobenzene (3c)

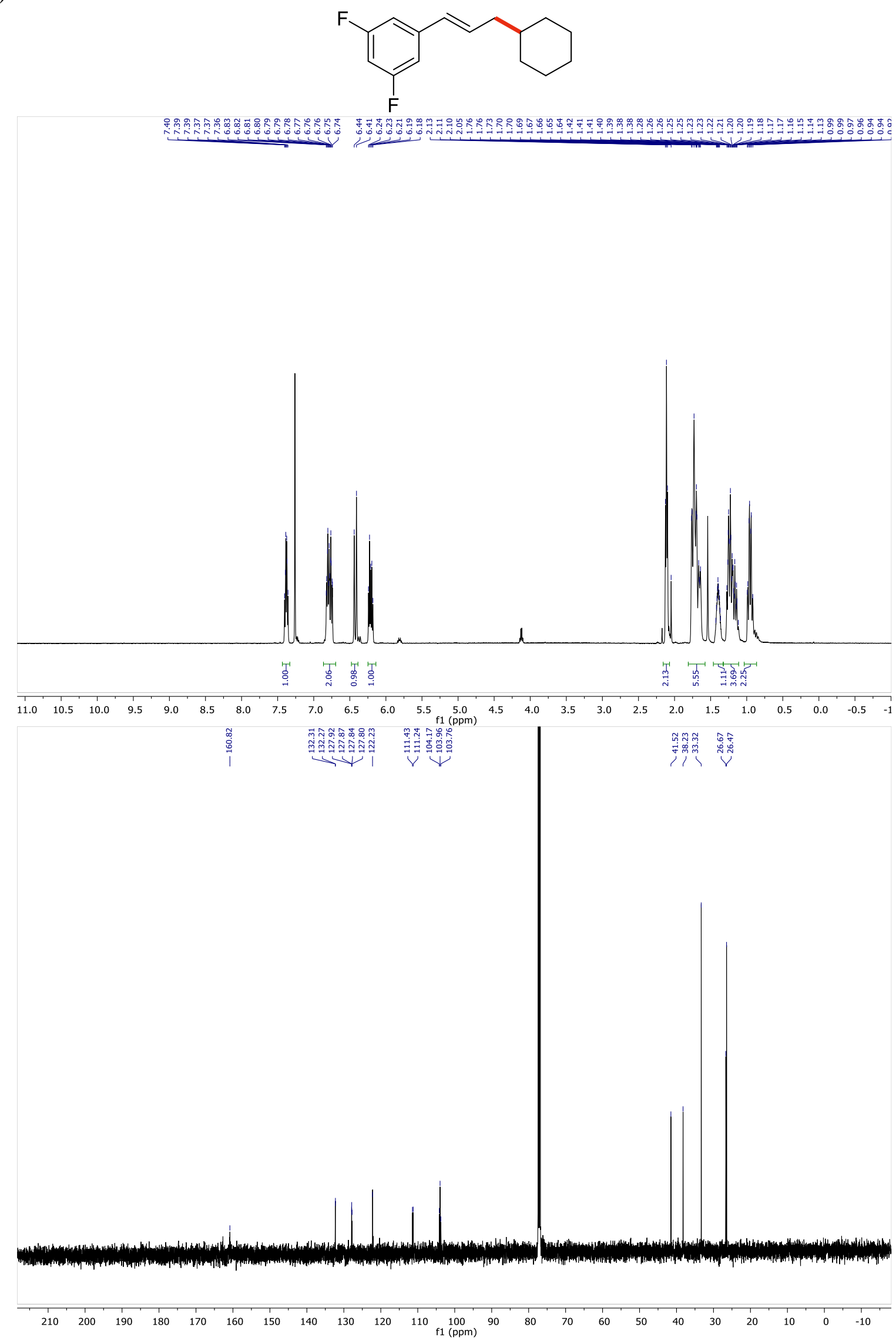




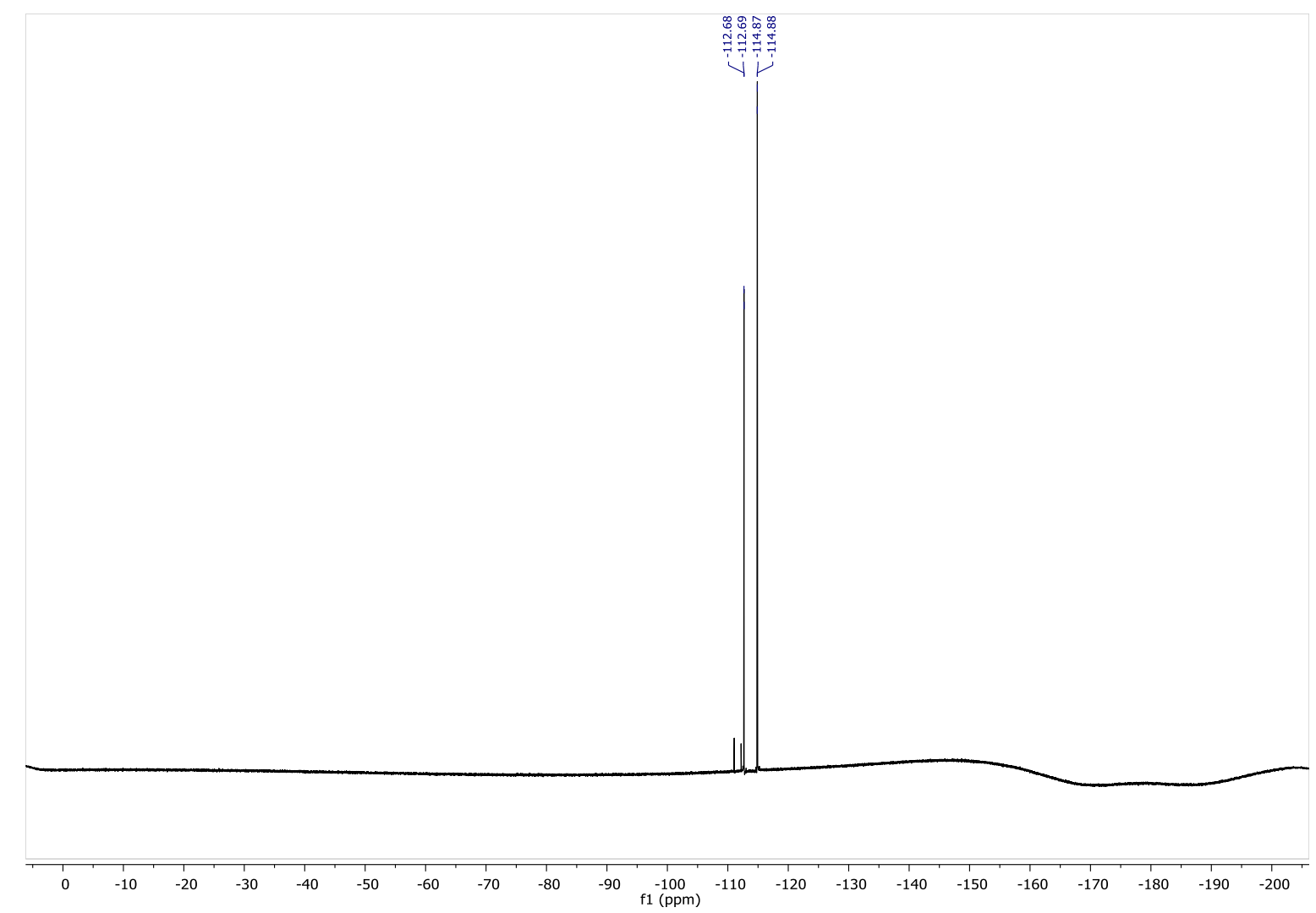


${ }^{1} \mathrm{H}-\mathrm{NMR}$ and ${ }^{13} \mathrm{C}-\mathrm{NMR}$ of (E)-1-Chloro-4-(4-methylpent-1-en-1-yl)benzene (3d)
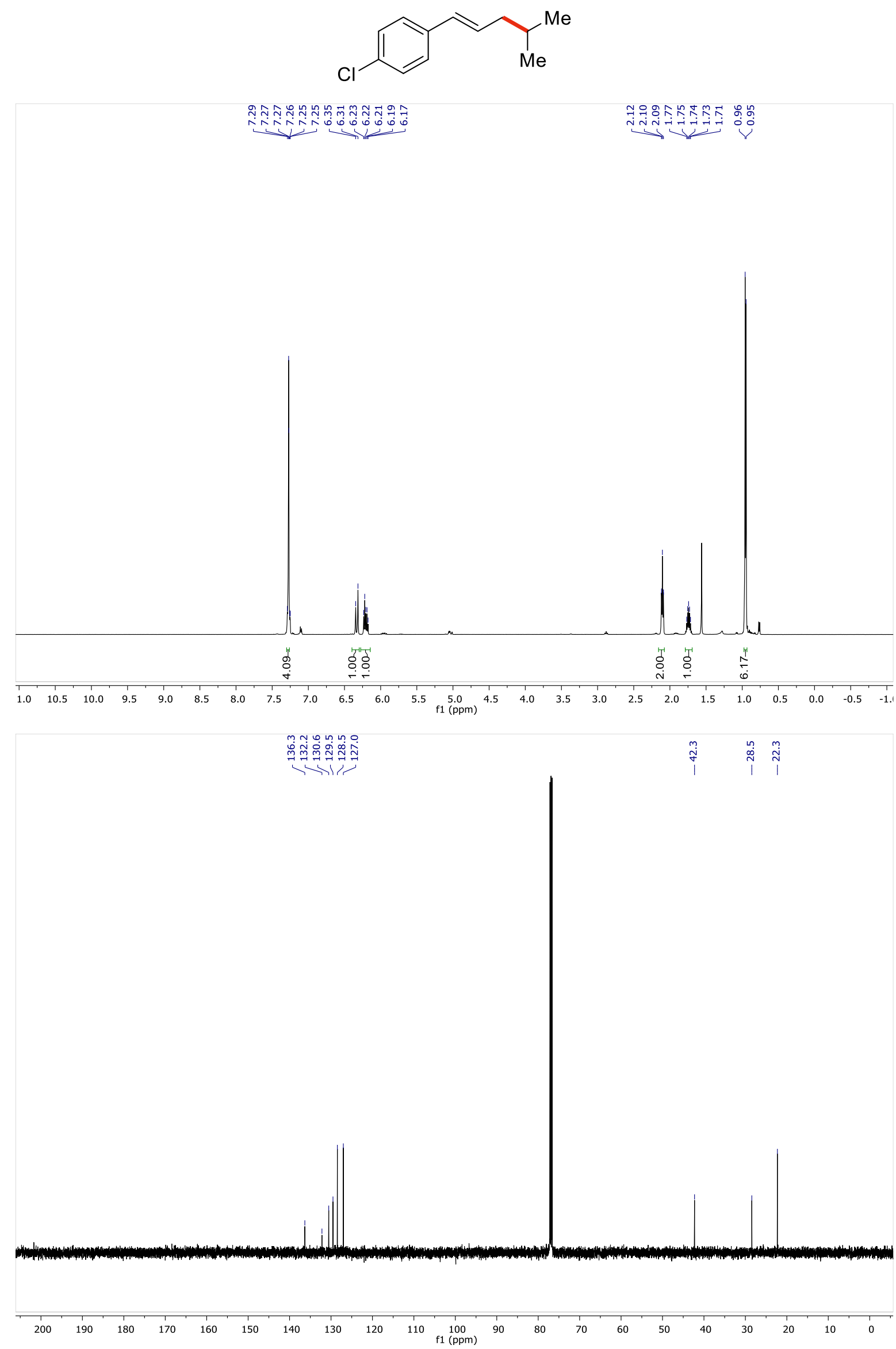
${ }^{1} \mathrm{H}-\mathrm{NMR}$ and ${ }^{13} \mathrm{C}-\mathrm{NMR}$ of (E)-1-(4-Methylpent-1-en-1-yl)-4-phenoxybenzene (3e)
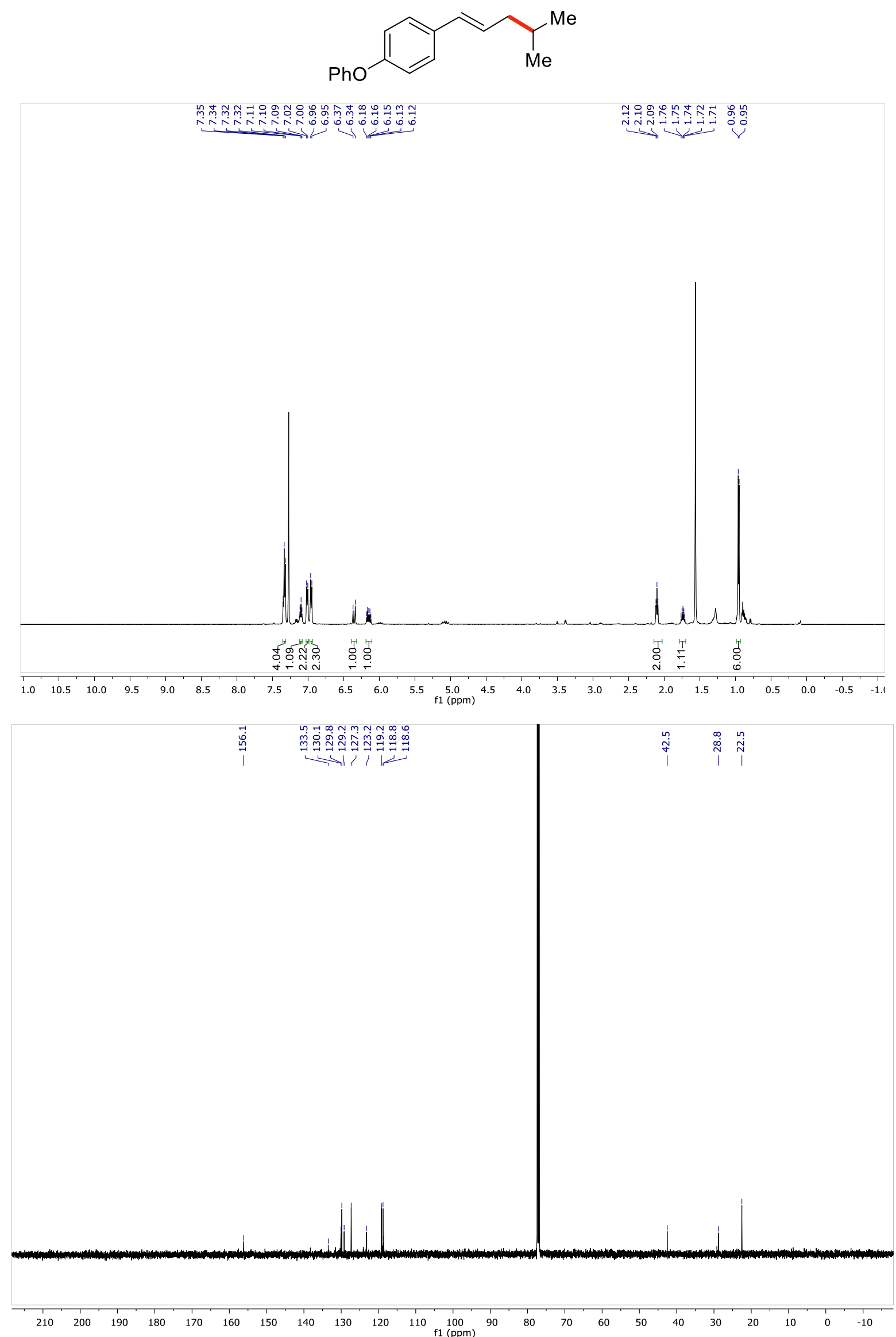
${ }^{1} \mathrm{H}-\mathrm{NMR}$ and ${ }^{13} \mathrm{C}-\mathrm{NMR}$ of (E)-6-(4-Methylpent-1-en-1-yl)-2,3-dihydrobenzo[b][1,4]dioxine (3f)
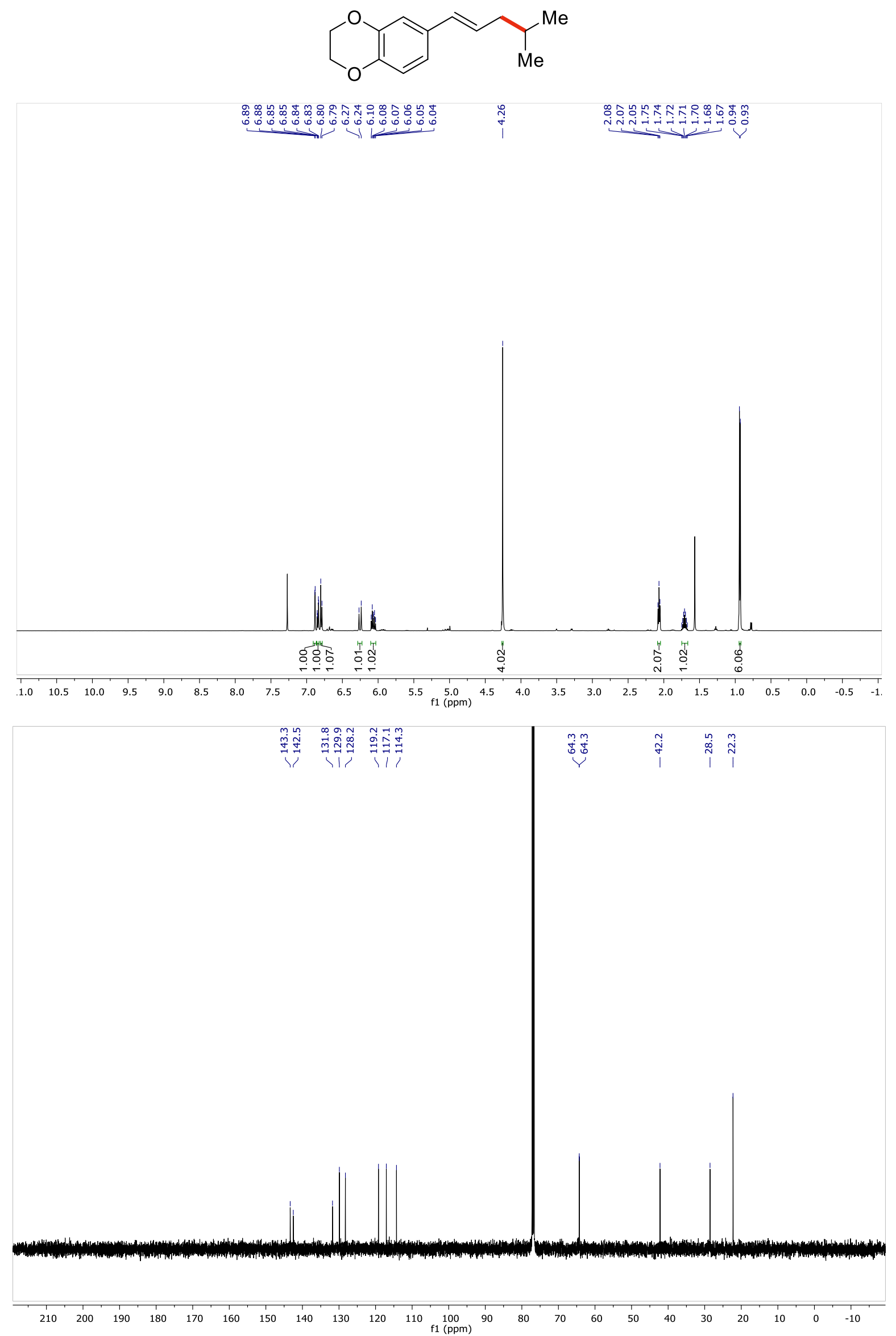
${ }^{1} \mathrm{H}-\mathrm{NMR}$ and ${ }^{13} \mathrm{C}-\mathrm{NMR}$ of (E)-1-Methoxy-4-(4-methylpent-1-en-1-yl)benzene (3g)<smiles>COc1ccc(/C=C/CC(C)C)cc1</smiles>

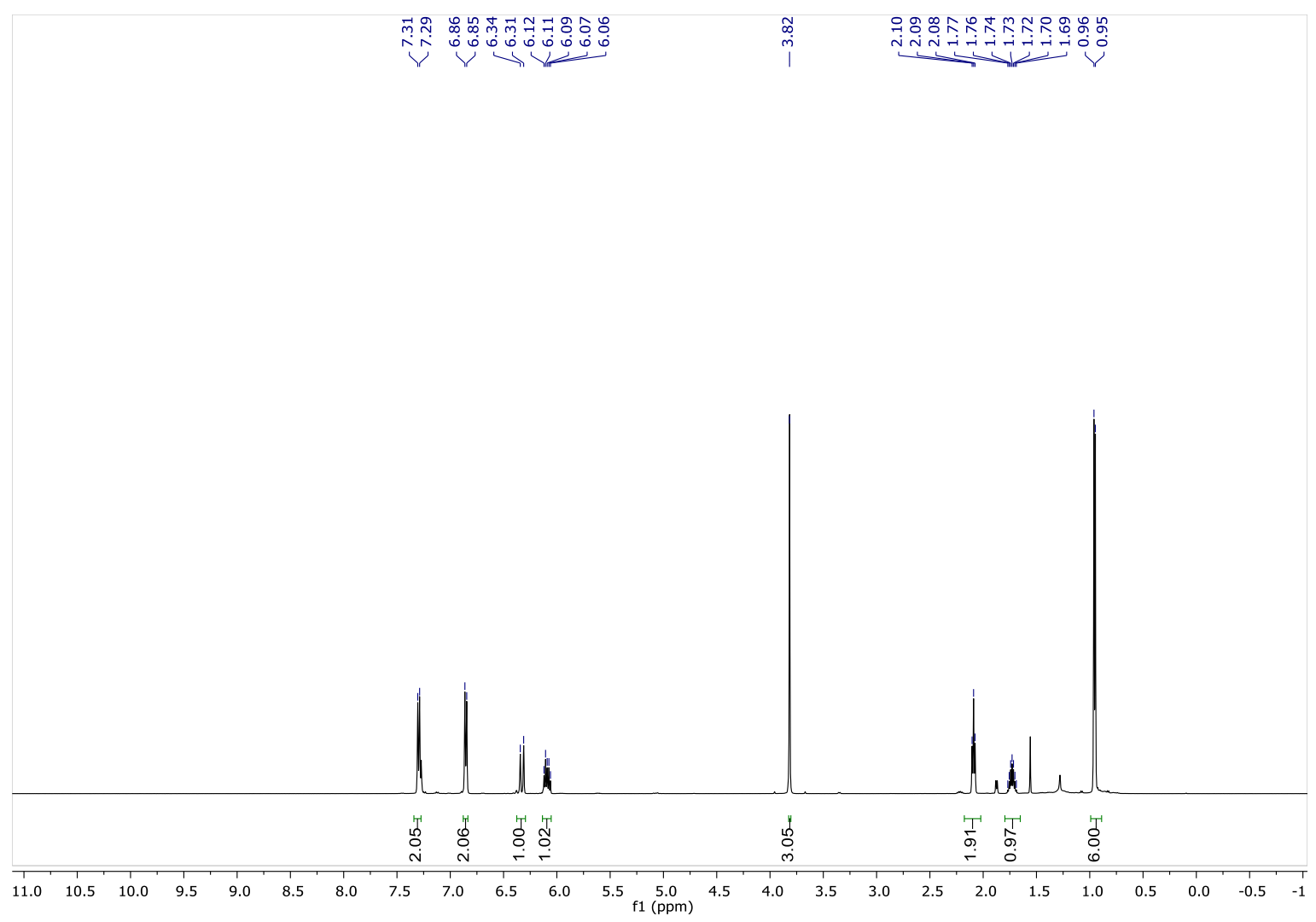

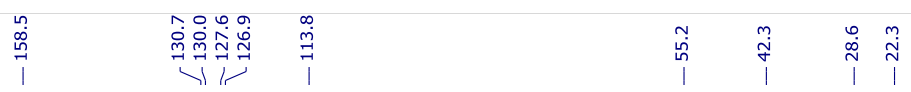

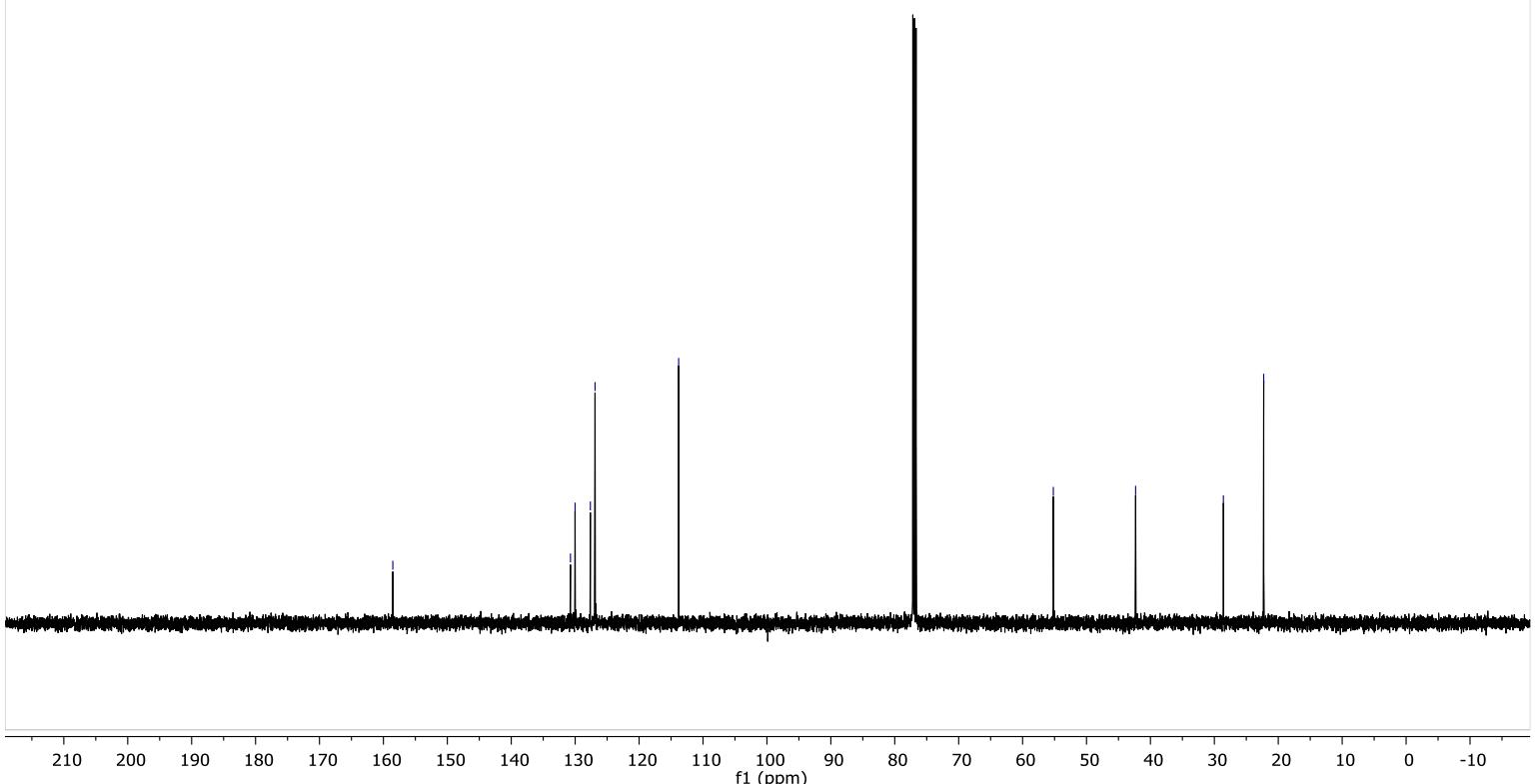


${ }^{1} \mathrm{H}-\mathrm{NMR}$ and ${ }^{13} \mathrm{C}-\mathrm{NMR}$ of (E)-(3-(Cyclohex-3-en-1-yl)prop-1-en-1-yl)benzene (3h)<smiles>C1=CCC(CC=Cc2ccccc2)CC1</smiles>

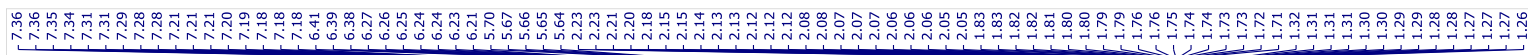
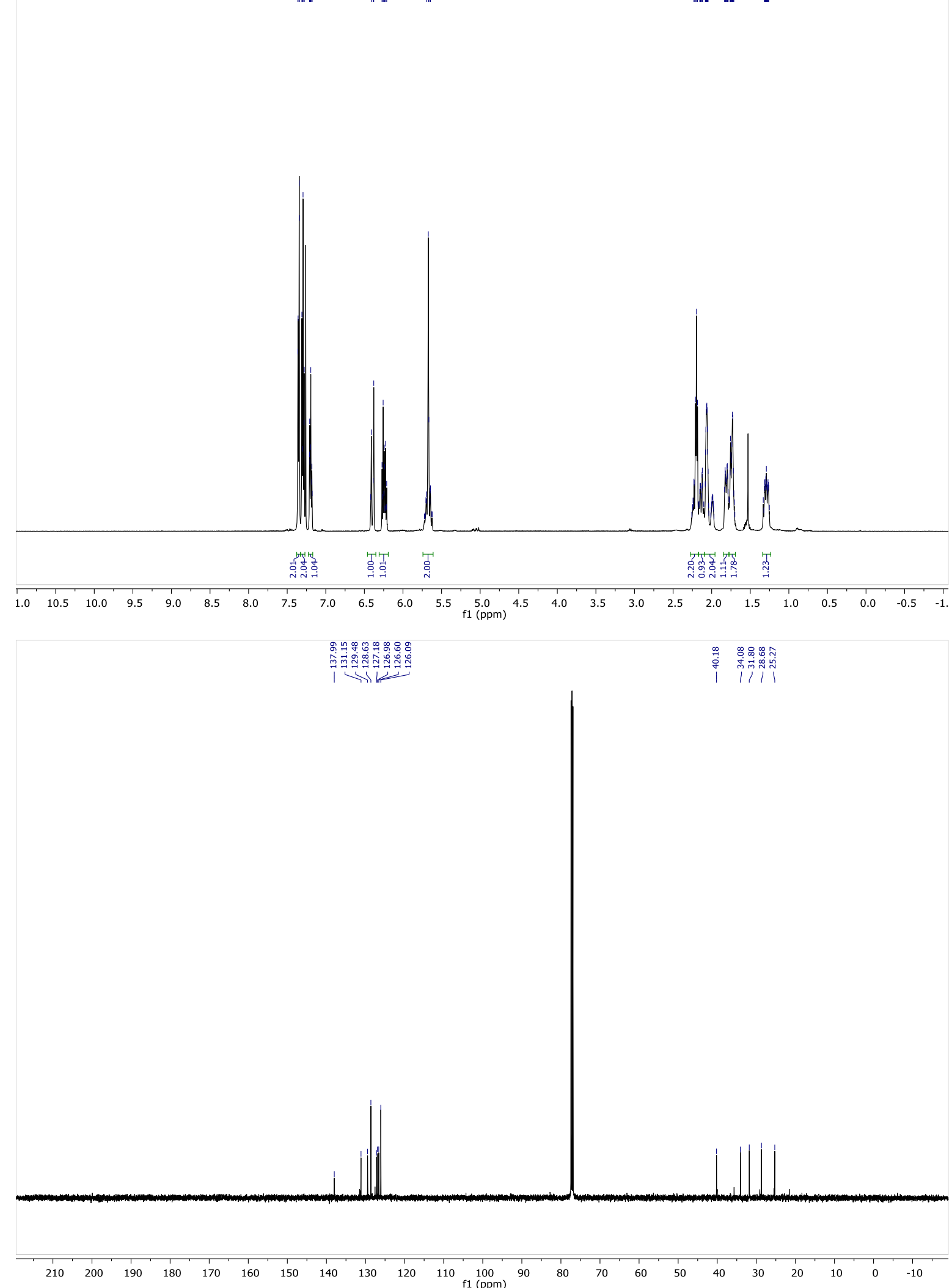
${ }^{1} \mathrm{H}-\mathrm{NMR}$ and ${ }^{13} \mathrm{C}-\mathrm{NMR}$ of 4-Cinnamyltetrahydro-2H-pyran (3i)

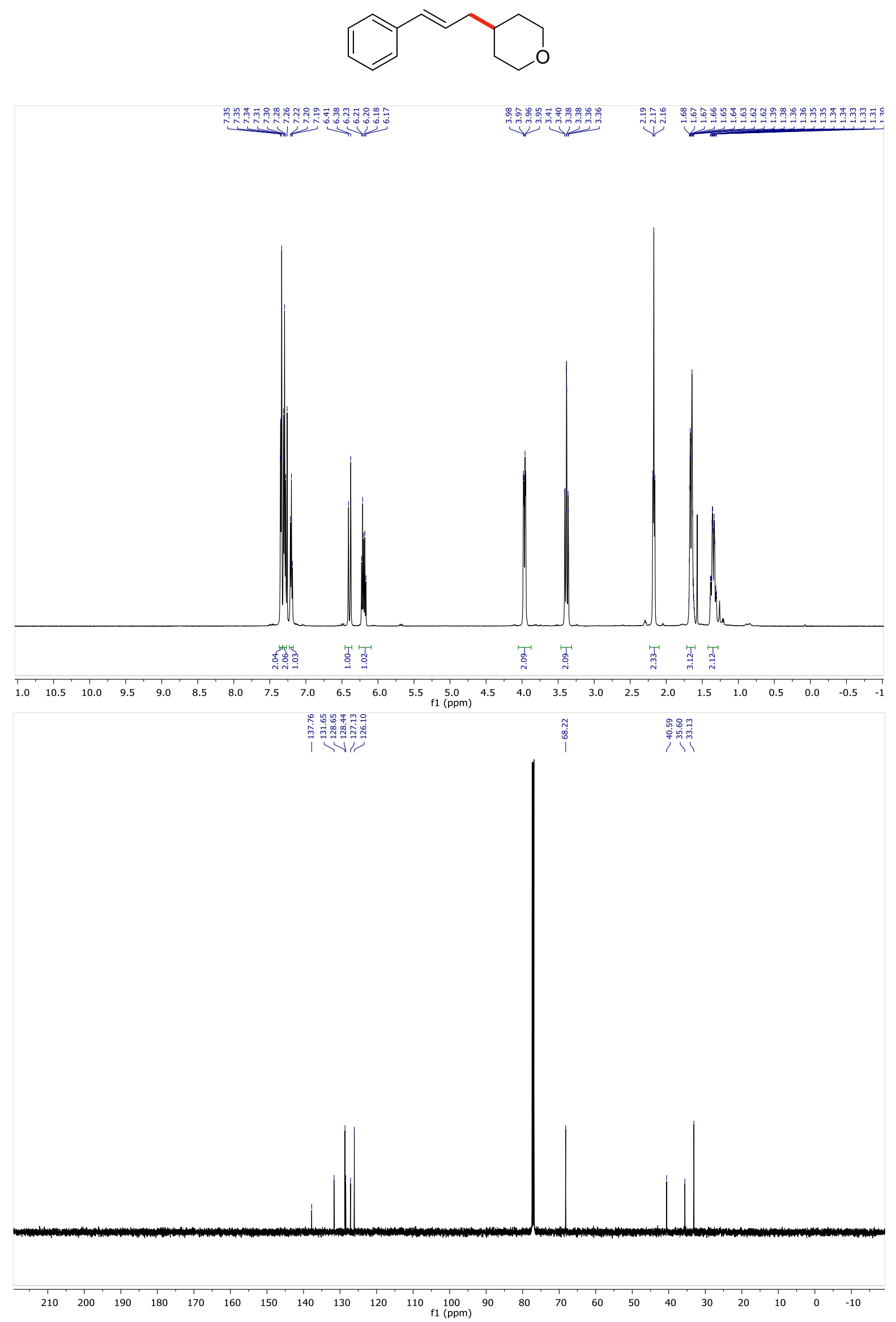


${ }^{1} \mathrm{H}-\mathrm{NMR}$ and ${ }^{13} \mathrm{C}-\mathrm{NMR}$ of $(E)$-(4-Methylhex-1-en-1-yl)benzene (3j)

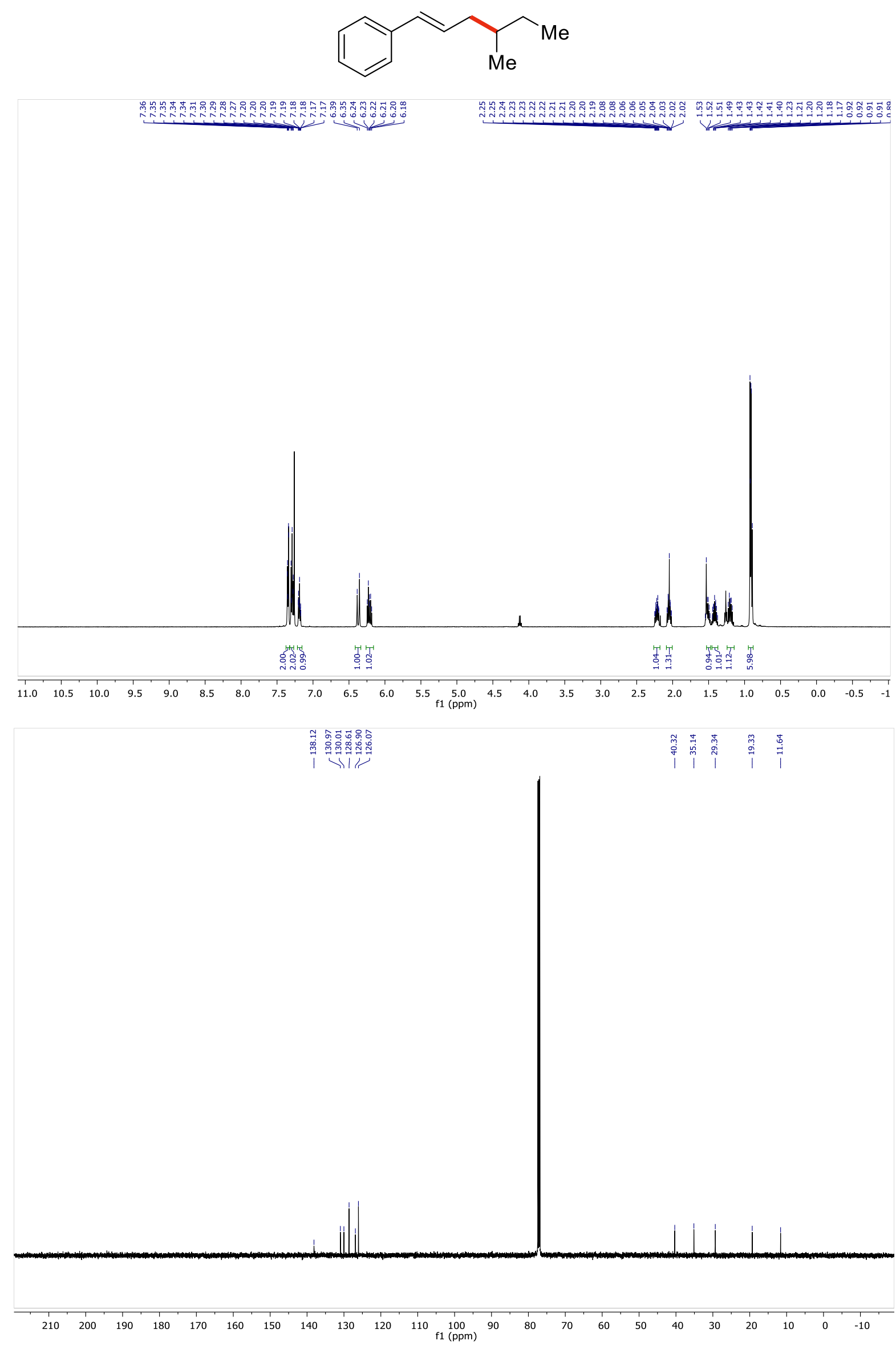


${ }^{1} \mathrm{H}-\mathrm{NMR}$ and ${ }^{13} \mathrm{C}-\mathrm{NMR}$ of Benzyl (E)-4-(3-(4-methoxyphenyl)allyl)piperidine-1-carboxylate(3k)<smiles>COc1ccc(/C=C/CC2CCN(C(=O)OCc3ccccc3)CC2)cc1</smiles>

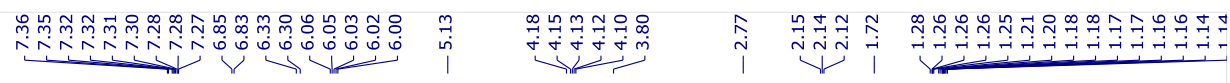
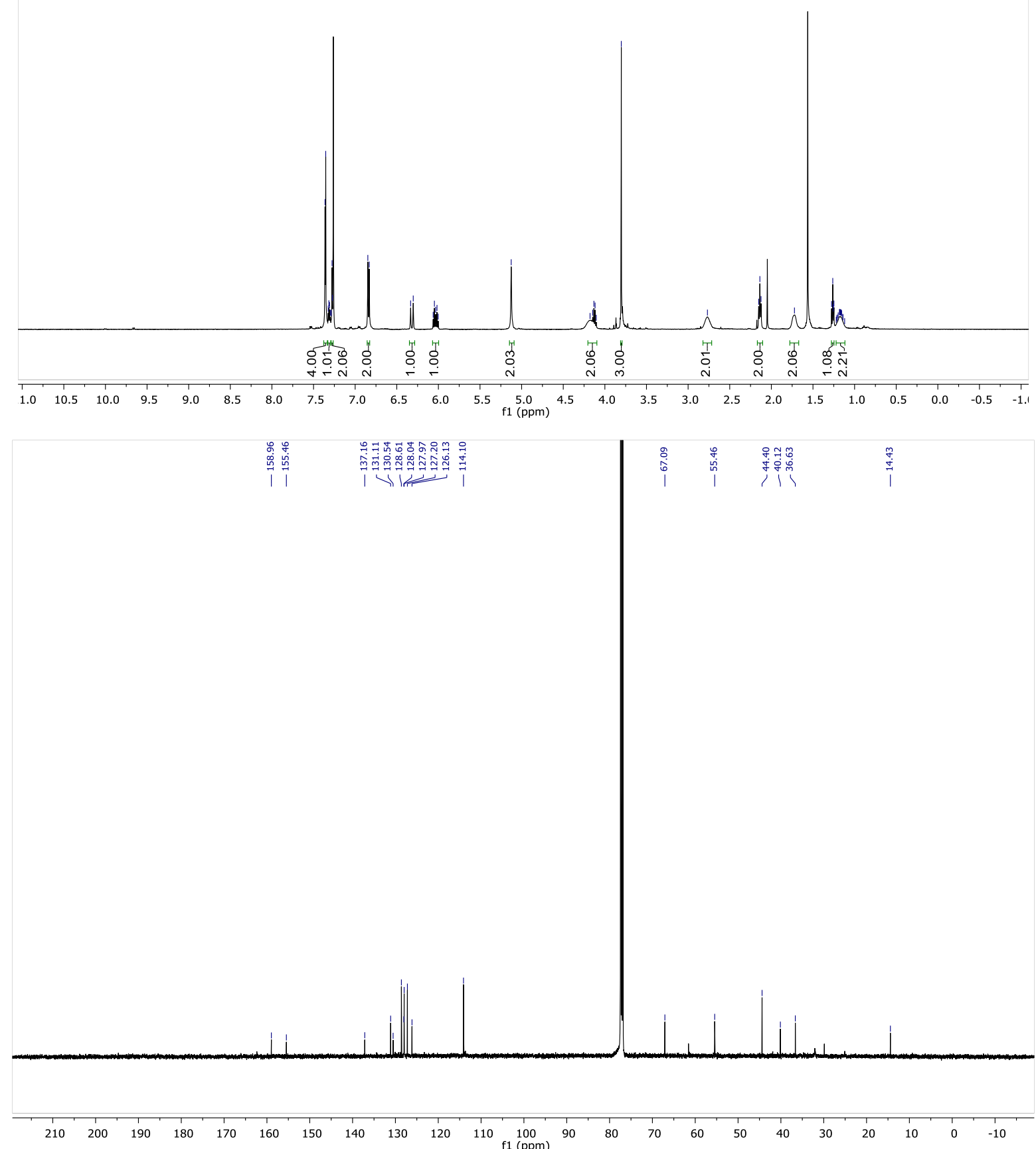
${ }^{1} \mathrm{H}-\mathrm{NMR}$ and ${ }^{13} \mathrm{C}-\mathrm{NMR}$ of (E)-4-Phenylbut-3-en-1-ol (3I)

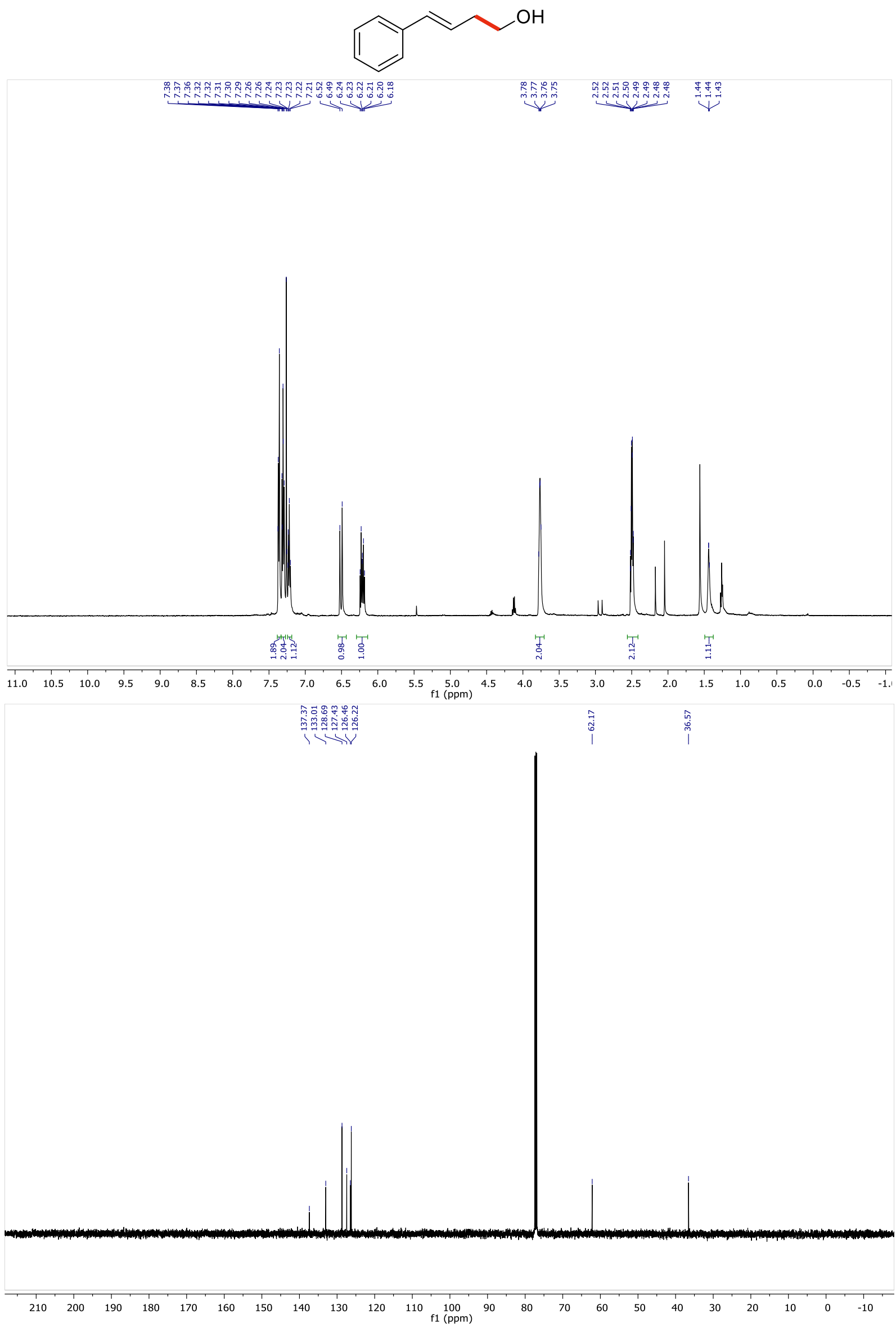


${ }^{1} \mathrm{H}-\mathrm{NMR}$ and $\quad{ }^{13} \mathrm{C}-\mathrm{NMR}$ of (3aR,4R,6R,6aR)-4-Cinnamyl-6-methoxy-2,2dimethyltetrahydrofuro[3,4-d][1,3]dioxole (4a)<smiles>COC1OC(C/C=C/c2ccccc2)C2OC(C)(C)OC12</smiles>
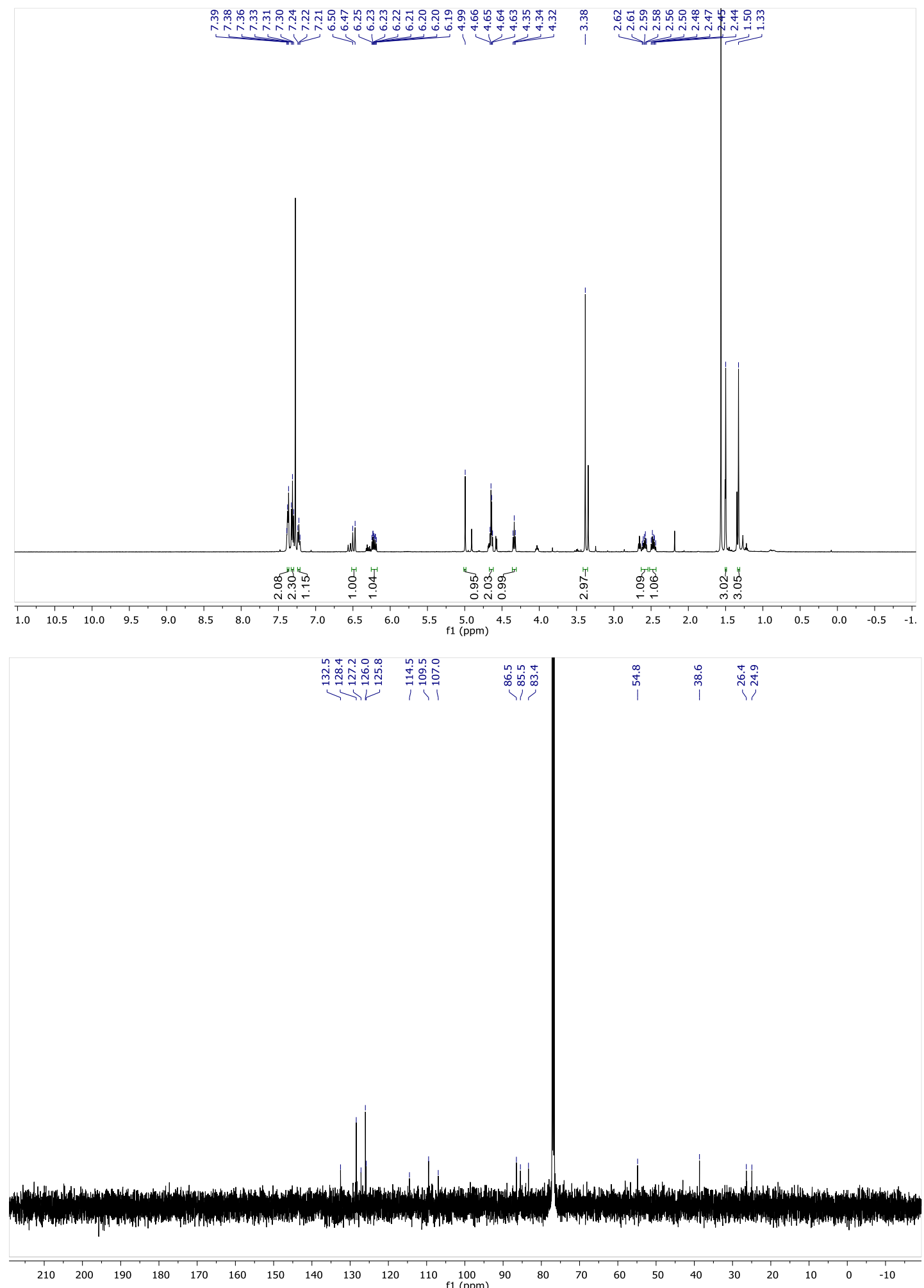
${ }^{1} \mathrm{H}-\mathrm{NMR}$ and ${ }^{13} \mathrm{C}-\mathrm{NMR}$ of (3aR,5aS,8bR)-5-Cinnamyl-2,2,7,7-tetramethyltetrahydro-5Hbis([1,3]dioxolo)[4,5-b:4',5'-d]pyran (4b)<smiles>CC1(C)O[C@H]2OC(C/C=C/c3ccccc3)[C@H]3OC(C)(C)O[C@H]3[C@H]2O1</smiles>

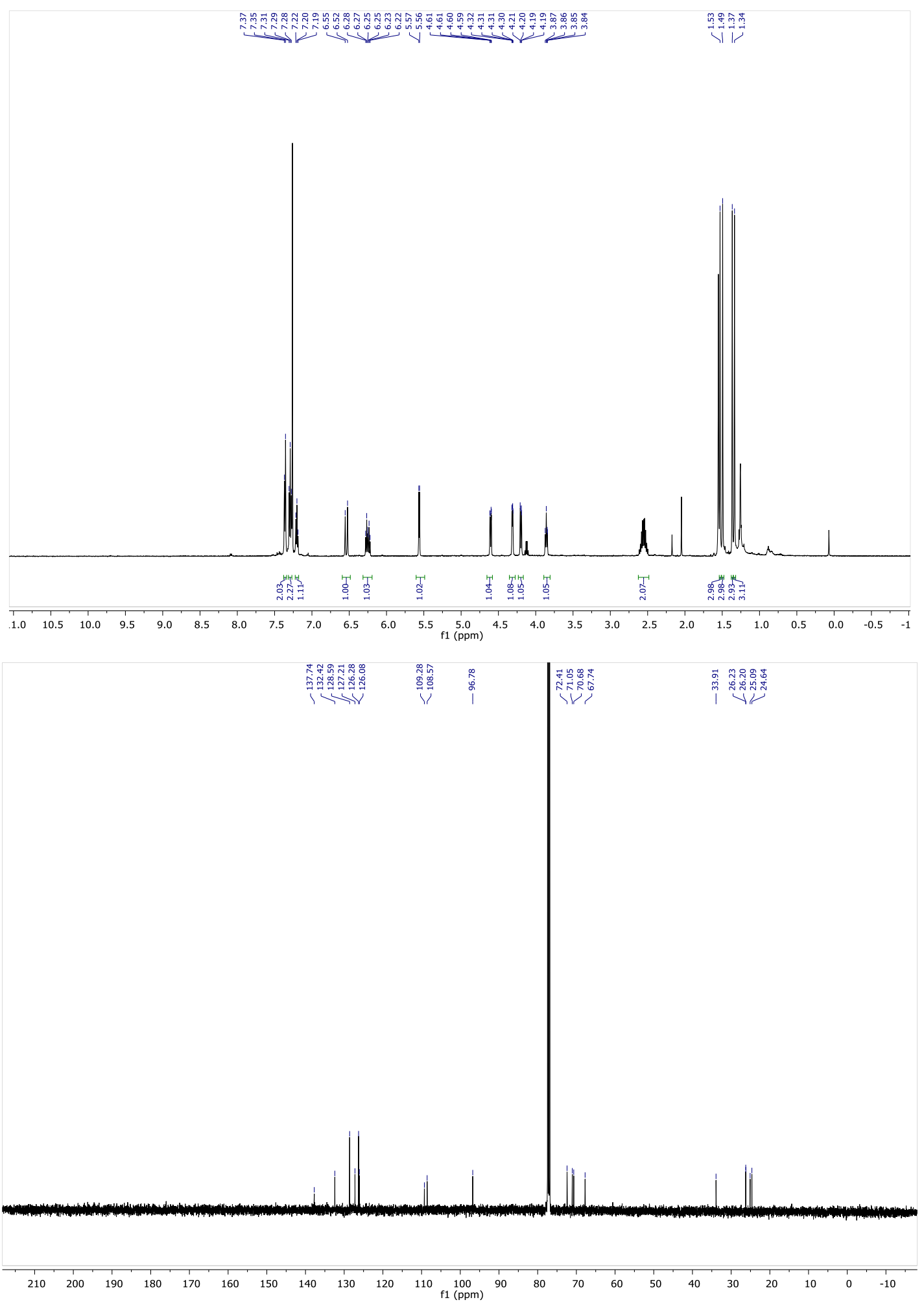


${ }^{1} \mathrm{H}$-NMR and ${ }^{13} \mathrm{C}-\mathrm{NMR}$ of (Cinnamylsulfonyl)benzene (5a)<smiles>O=S(=O)(C/C=C/c1ccccc1)c1ccccc1</smiles>
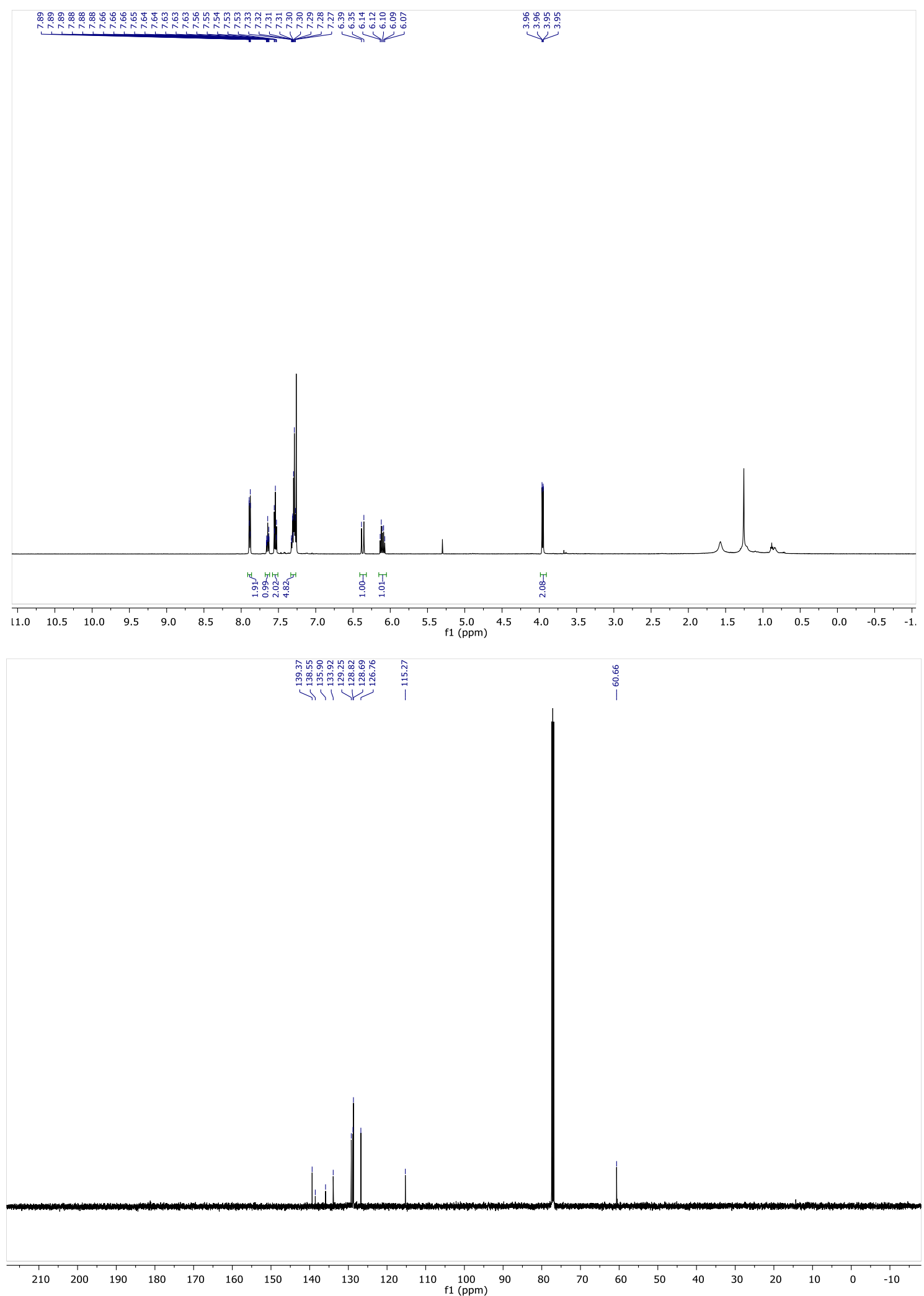
${ }^{1} \mathrm{H}-\mathrm{NMR}$ and ${ }^{13} \mathrm{C}$-NMR of 1-(Cinnamylsulfonyl)-4-methylbenzene (5b)
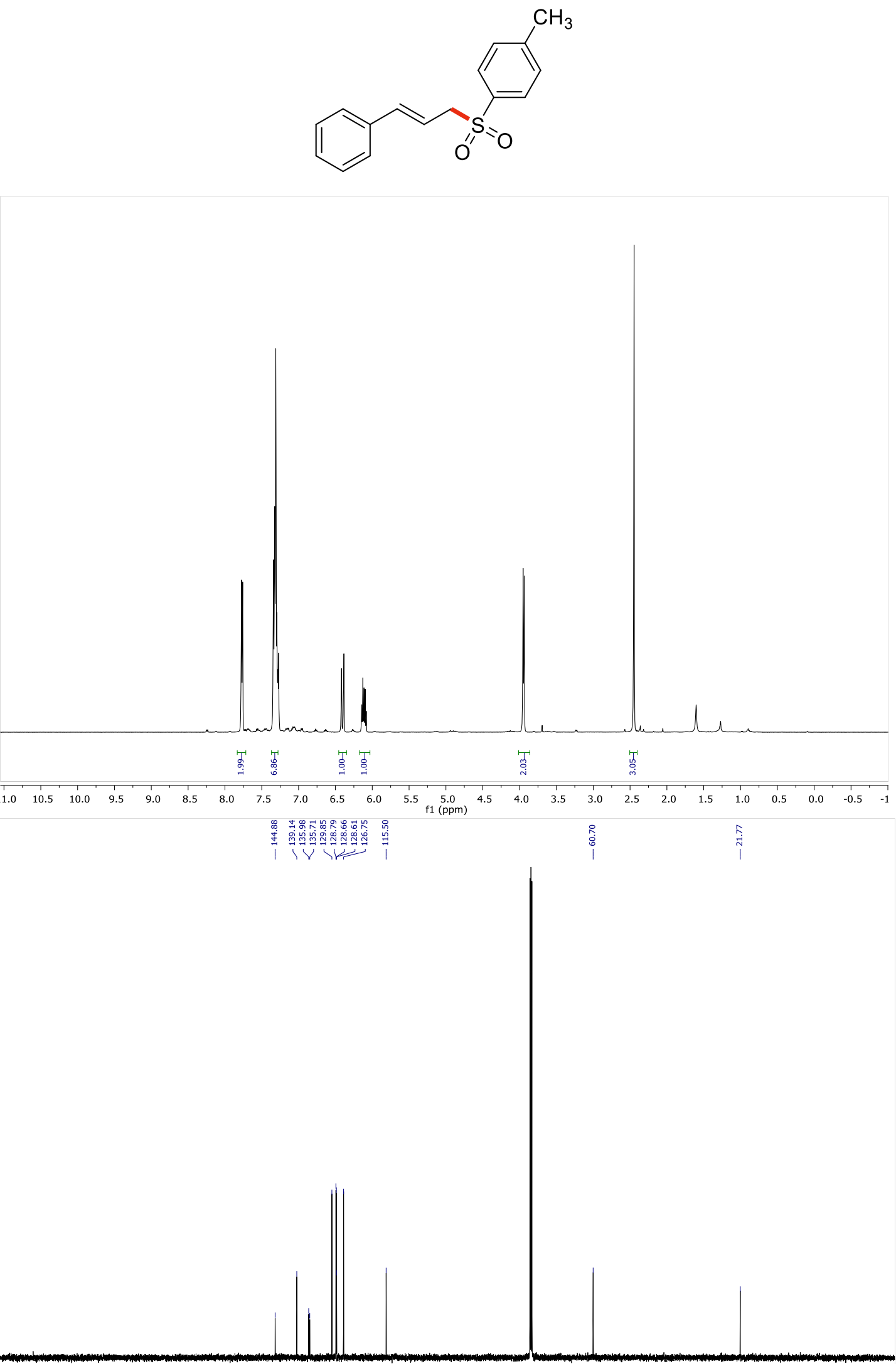

$\begin{array}{llllllllllllllllllllllllllllllllllll}210 & 200 & 190 & 180 & 170 & 160 & 150 & 140 & 130 & 120 & 110 & 100 & 90 & 80 & 70 & 60 & 50 & 40 & 30 & 20 & 10 & 0 & -10\end{array}$ 
${ }^{1} \mathrm{H}-\mathrm{NMR},{ }^{13} \mathrm{C}-\mathrm{NMR}$ and ${ }^{19} \mathrm{~F}-\mathrm{NMR}$ of (E)-1-Fluoro-4-(3-(phenylsulfonyl)prop-1-en-1-yl)benzene (5c)

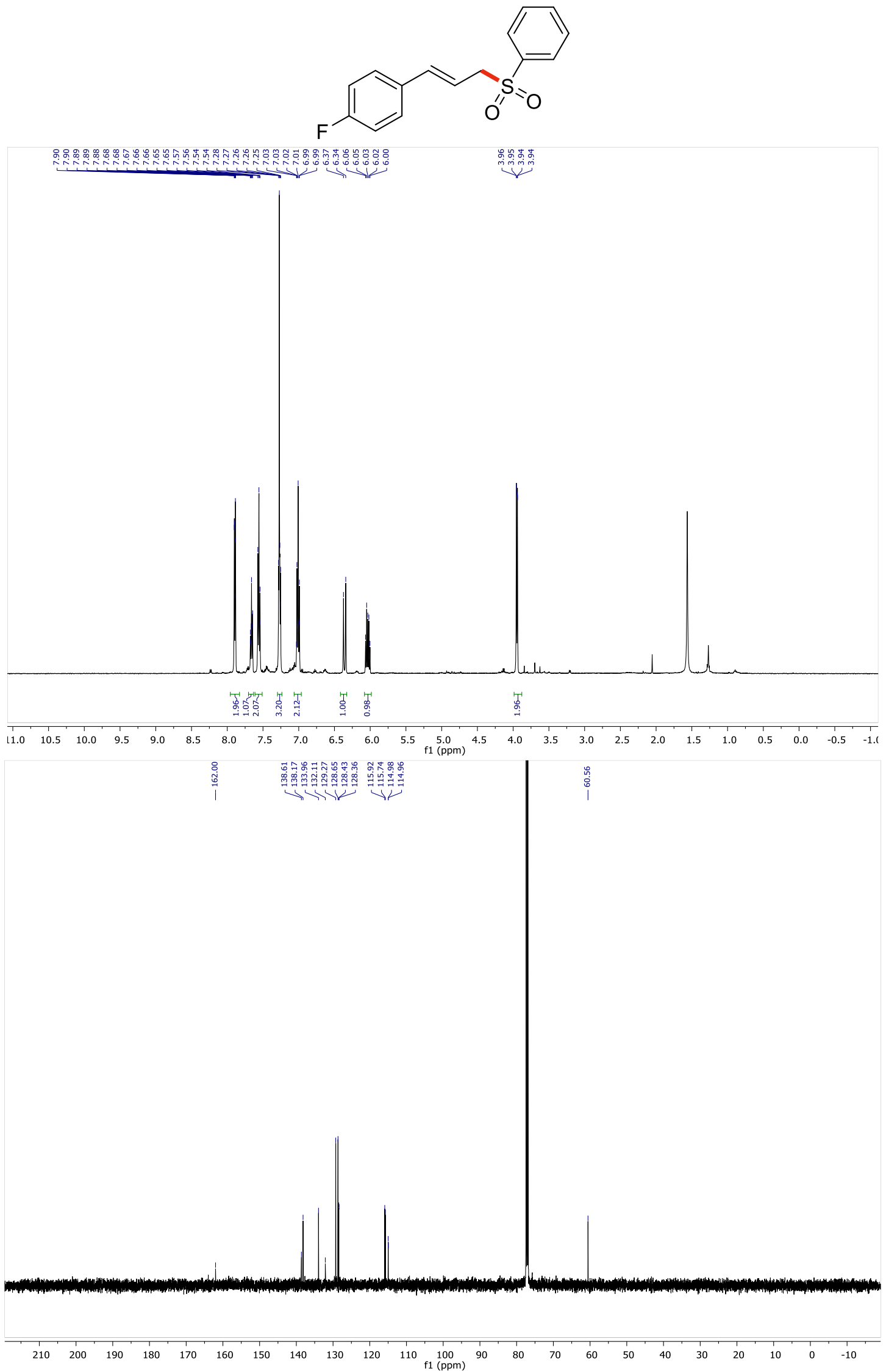




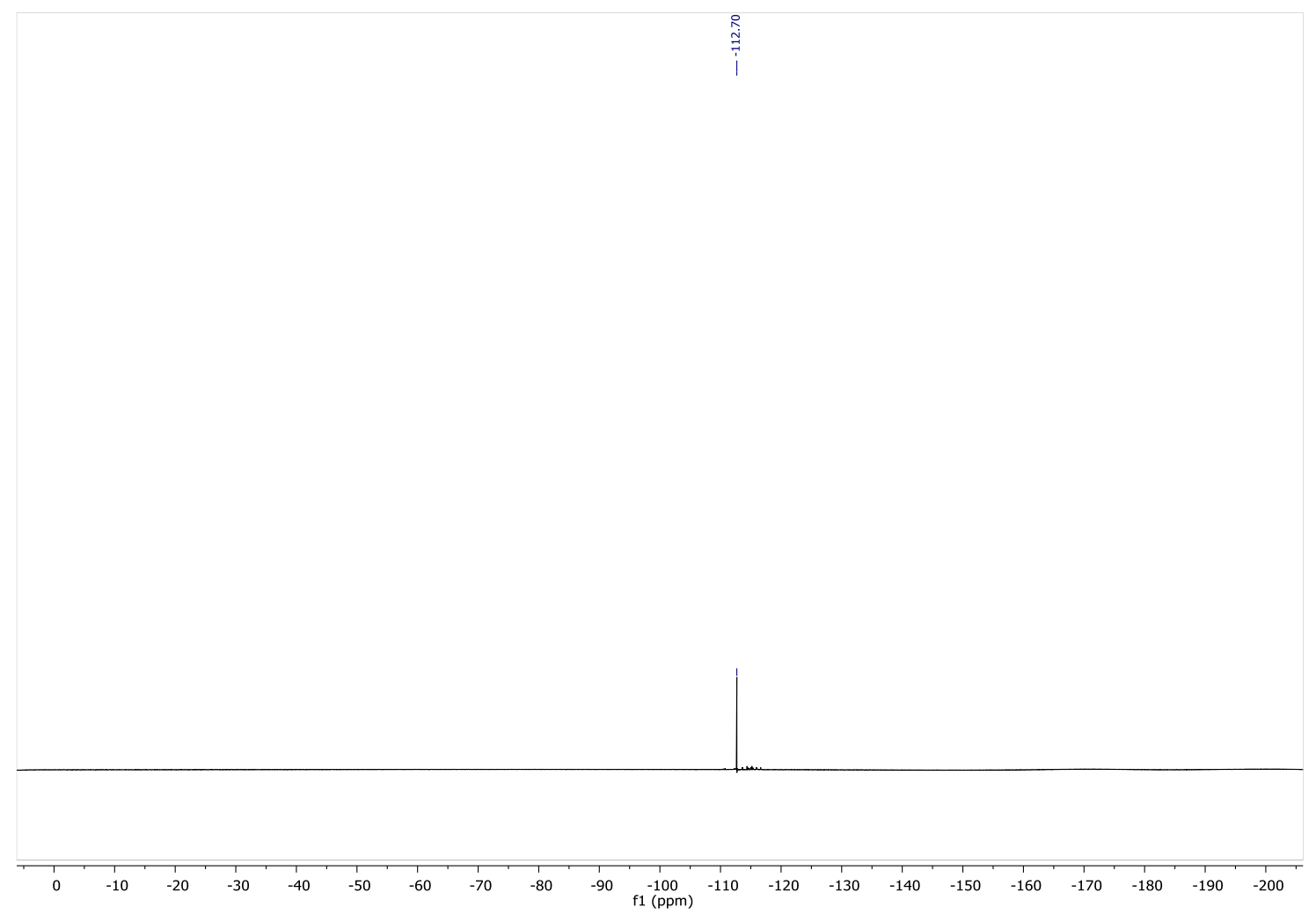


${ }^{1} \mathrm{H}-\mathrm{NMR}$ and ${ }^{13} \mathrm{C}-\mathrm{NMR}$ of (E)-2-(3-(Phenylsulfonyl)prop-1-en-1-yl)naphthalene (5d)
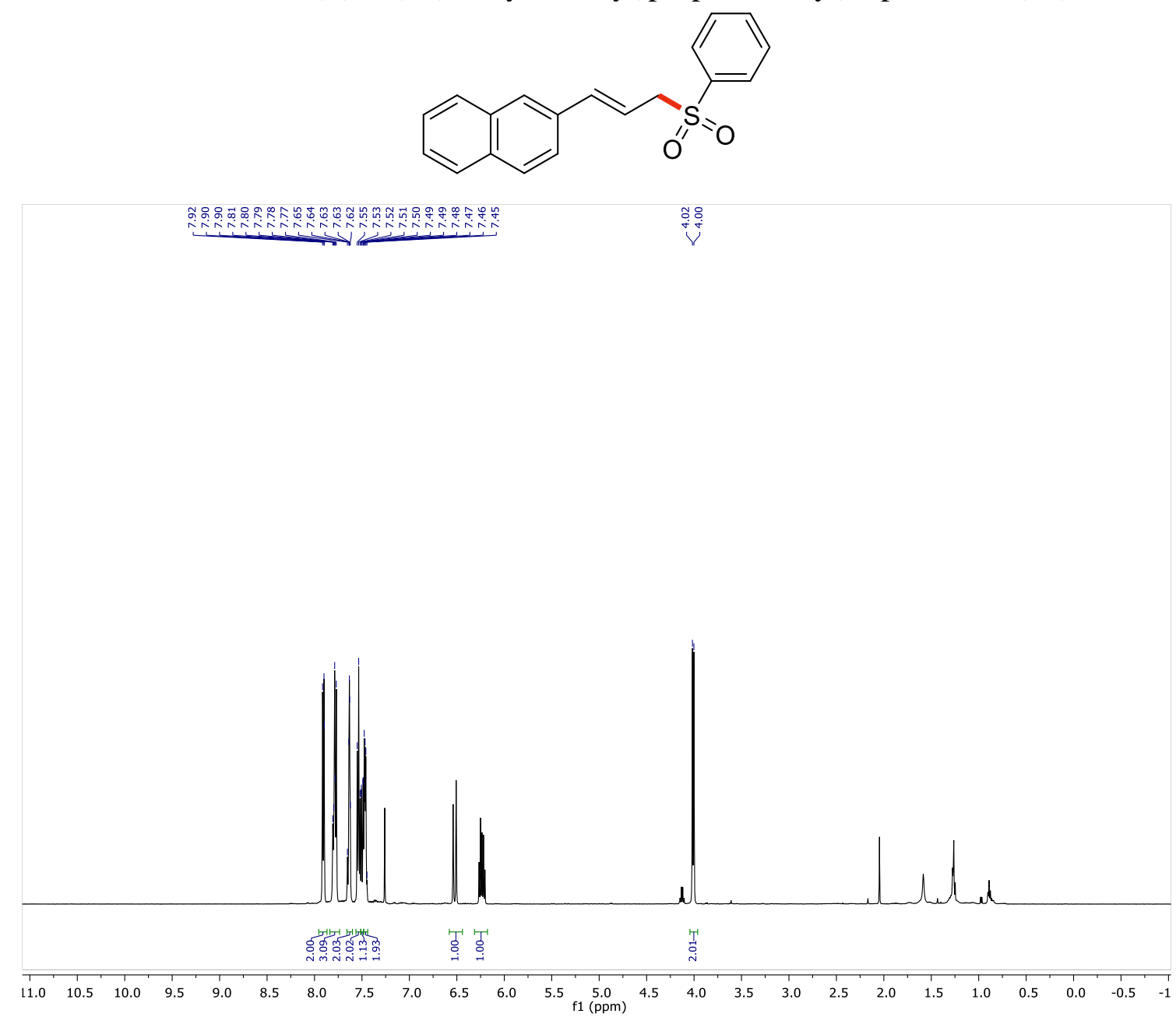


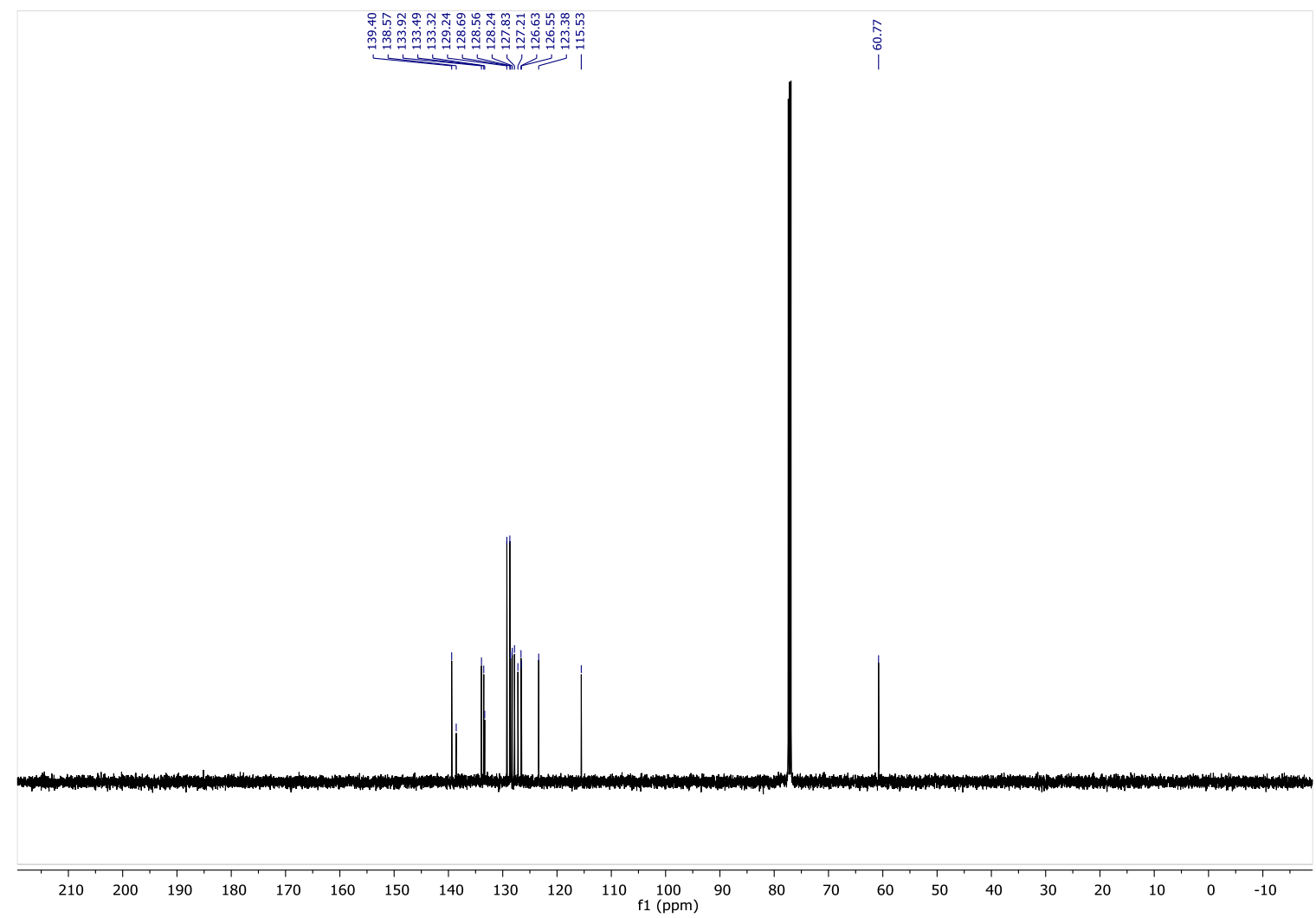

${ }^{1} \mathrm{H}-\mathrm{NMR}$ and ${ }^{13} \mathrm{C}-\mathrm{NMR}$ of (E)-1-Methoxy-4-(3-(phenylsulfonyl)prop-1-en-1-yl)benzene (5e)

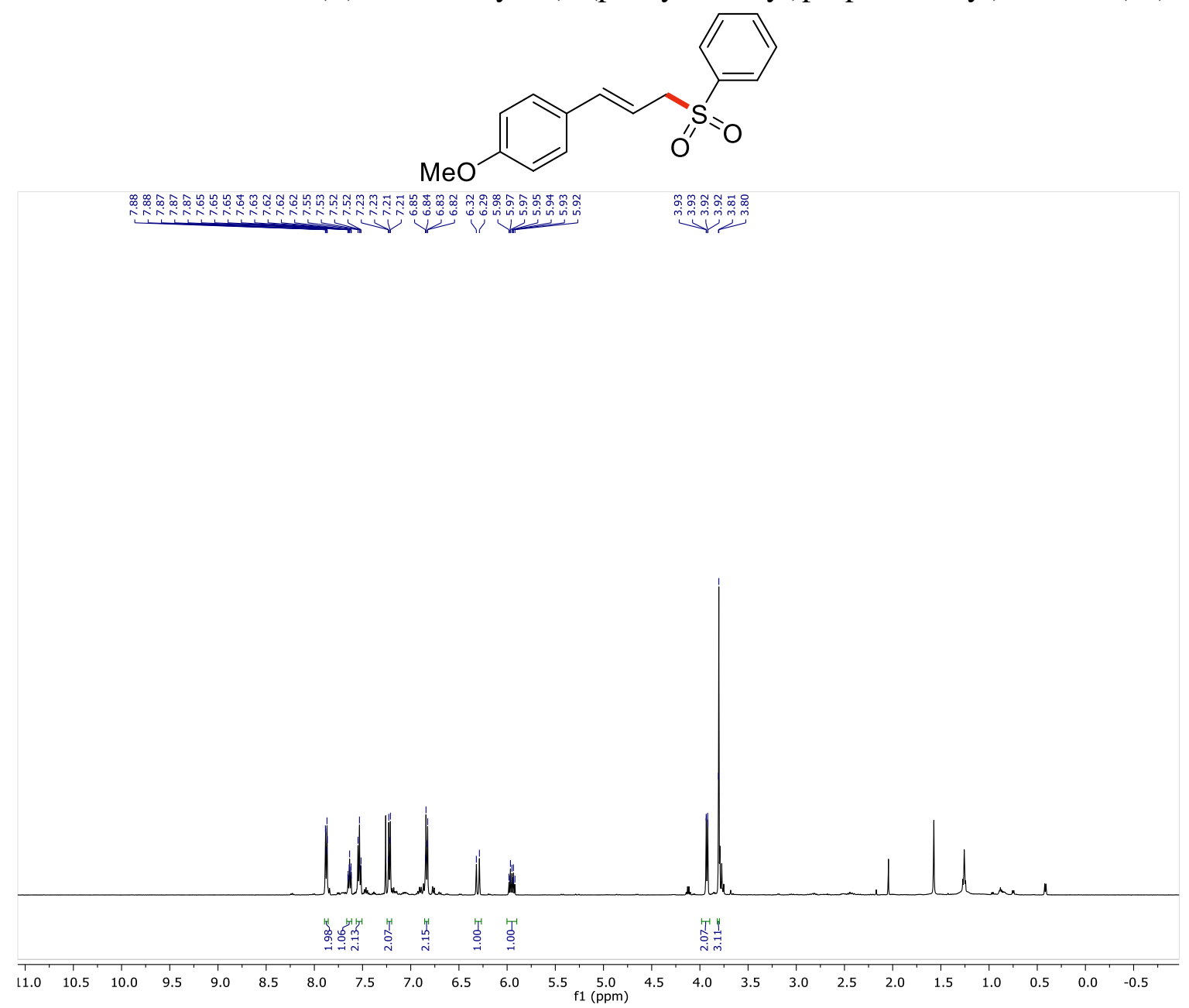




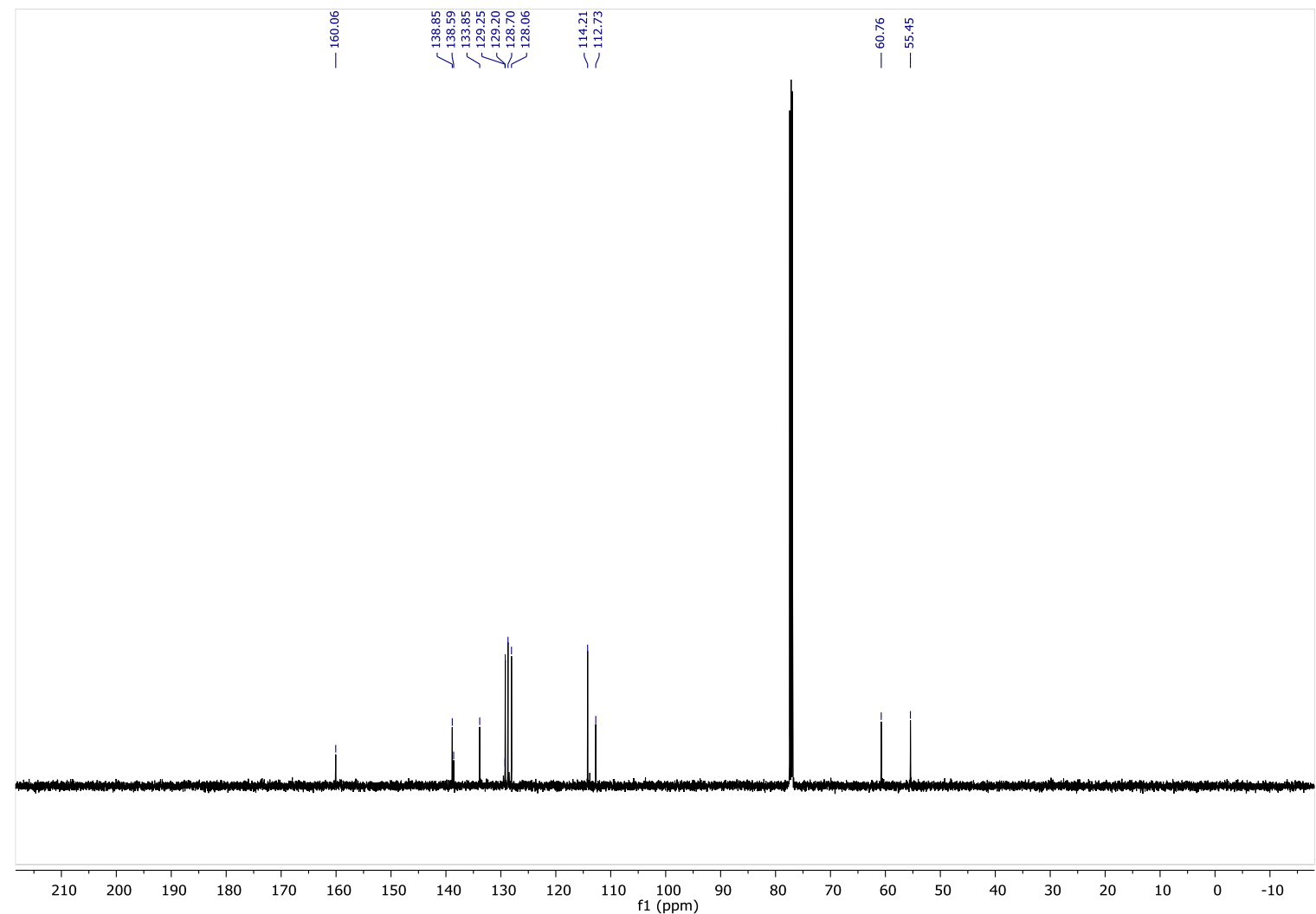

${ }^{1} \mathrm{H}-\mathrm{NMR}$ and ${ }^{13} \mathrm{C}-\mathrm{NMR}$ of $(E)-((5,9-D i m e t h y l d e c a-2,8$-dien-1-yl)sulfonyl)benzene (5f)<smiles>CC(C)=CCCC(C)CC=CCS(=O)(=O)c1ccccc1</smiles>

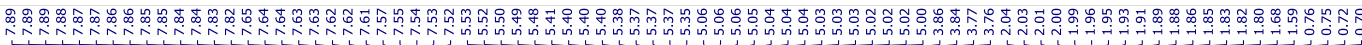

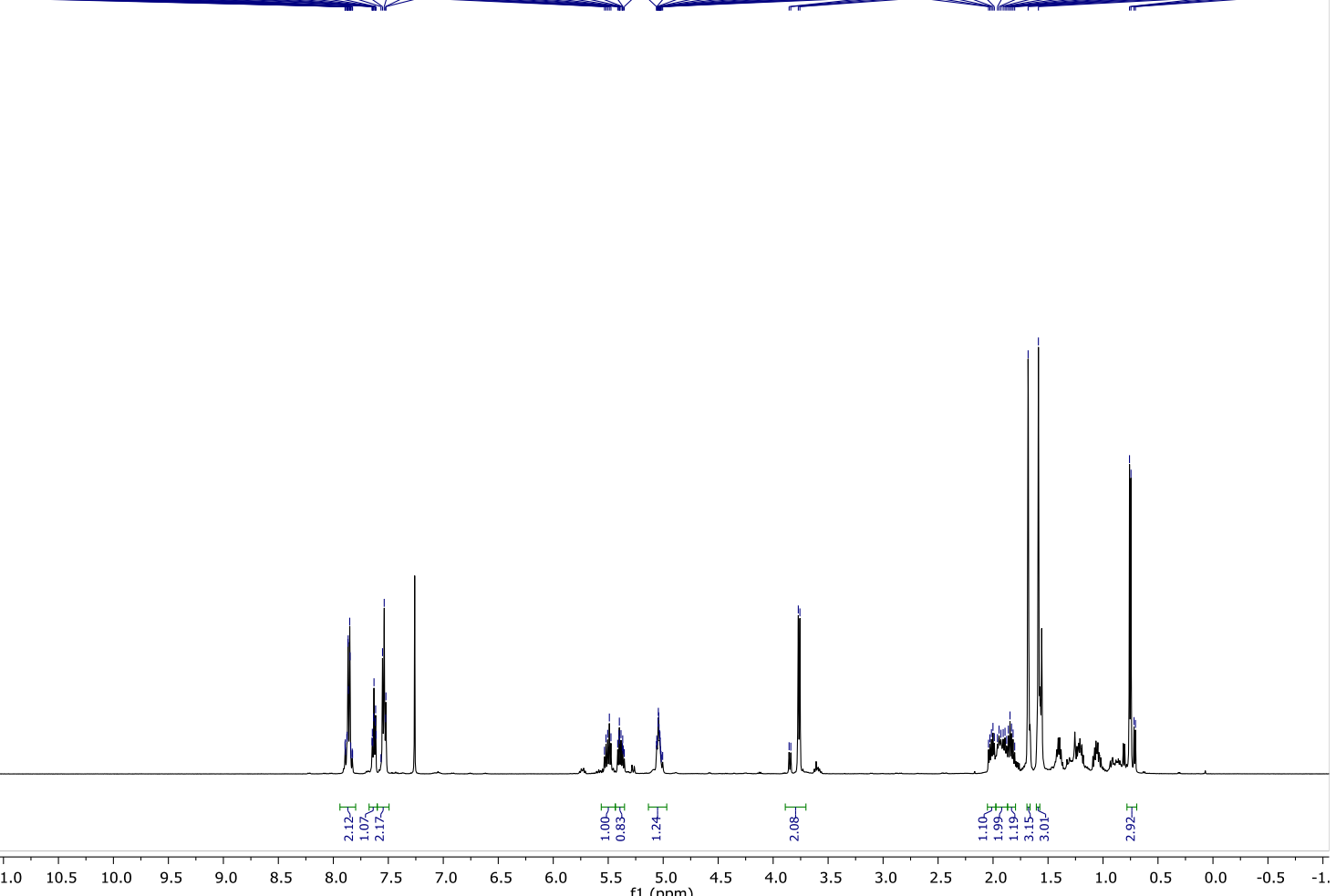




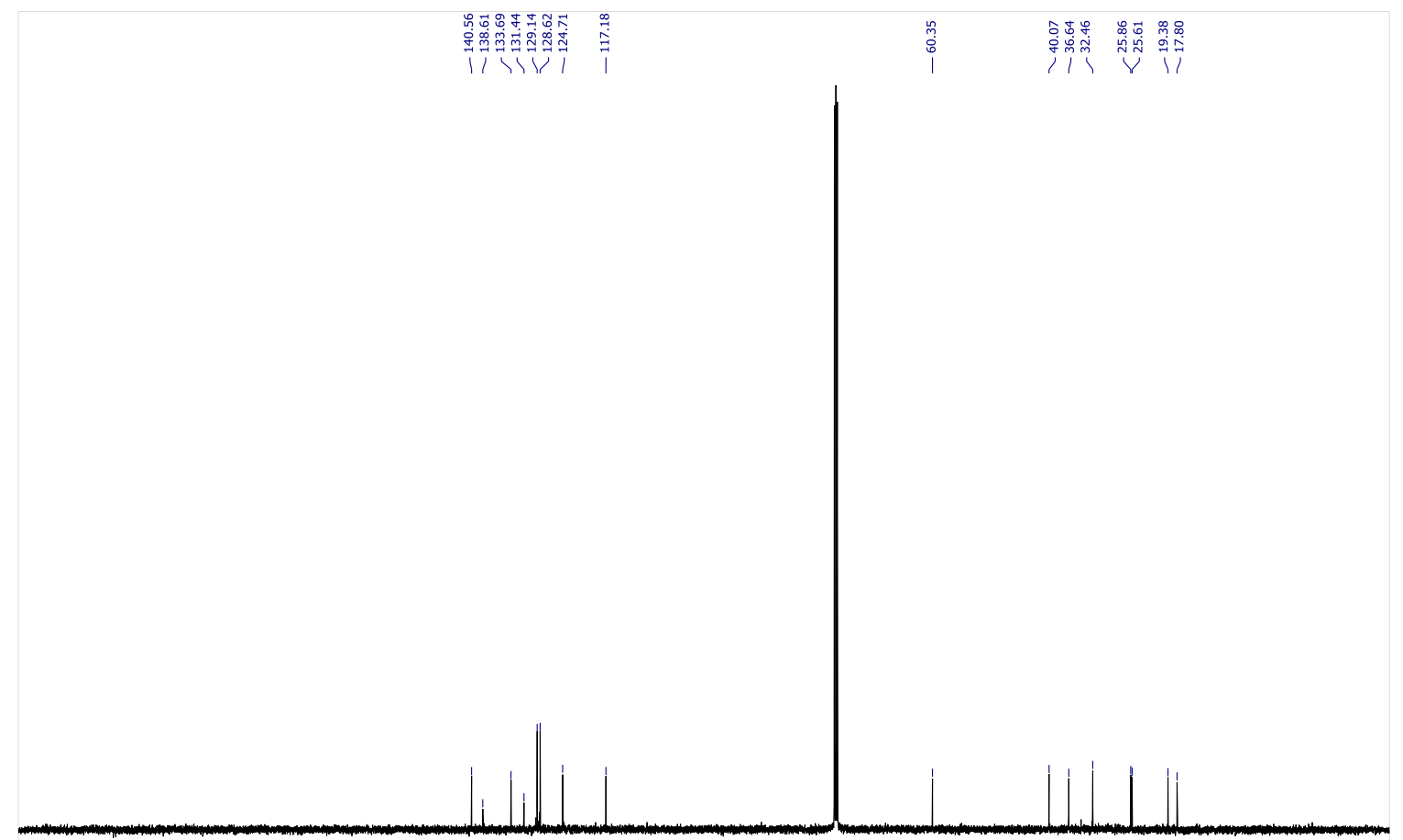

$\begin{array}{lllllllllllllllllllllll}210 & 200 & 190 & 180 & 170 & 160 & 150 & 140 & 130 & 120 & 110 & \begin{array}{c}100 \\ \mathrm{f} 1(\mathrm{ppm})\end{array} & 90 & 80 & 70 & 60 & 50 & 40 & 30 & 20 & 10 & 0 & -10\end{array}$

${ }^{1} \mathrm{H}-\mathrm{NMR}$ and ${ }^{13} \mathrm{C}-\mathrm{NMR}$ of (E)-Methyl (3-phenylallyl-1-d) carbonate (S1-d)

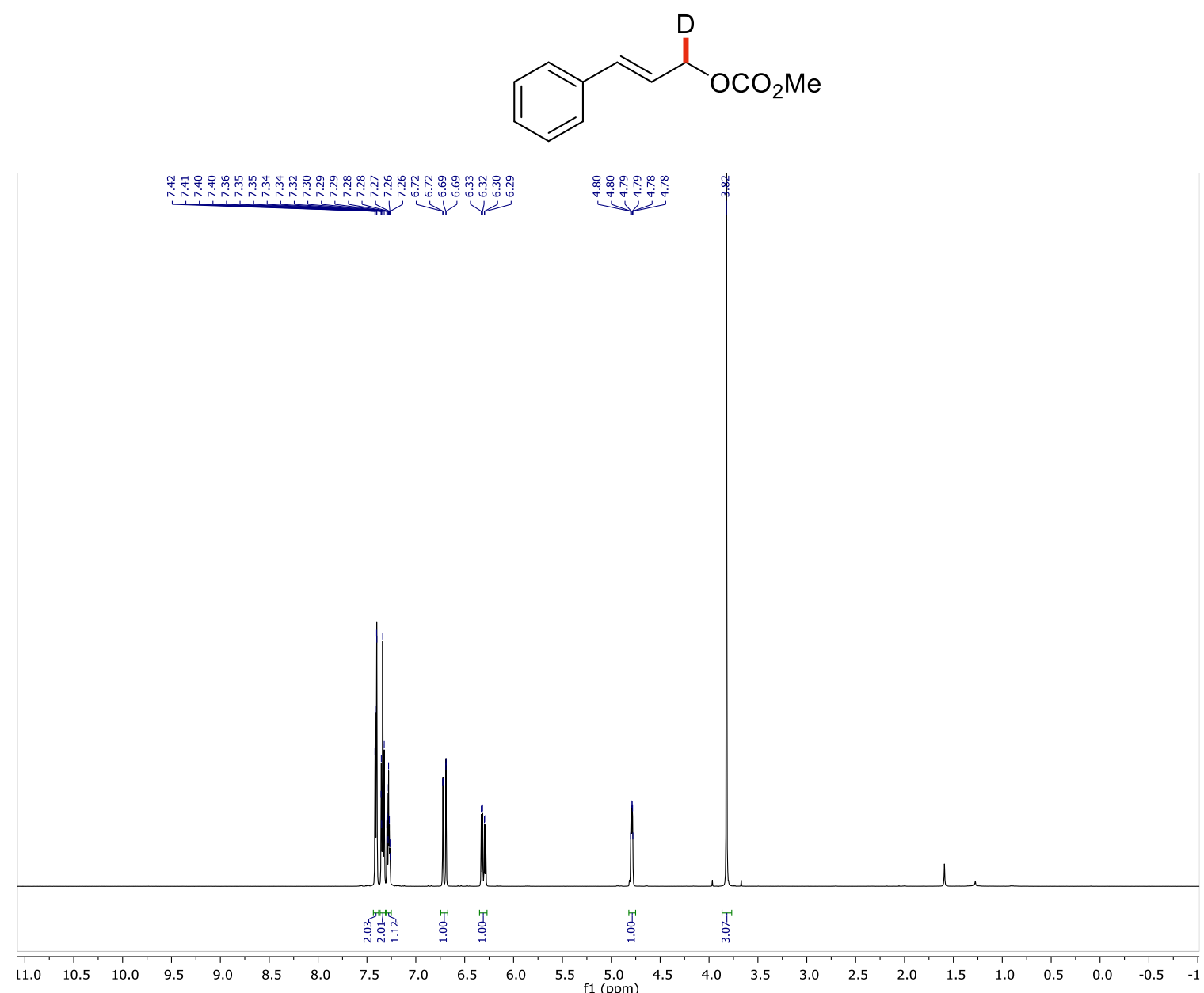




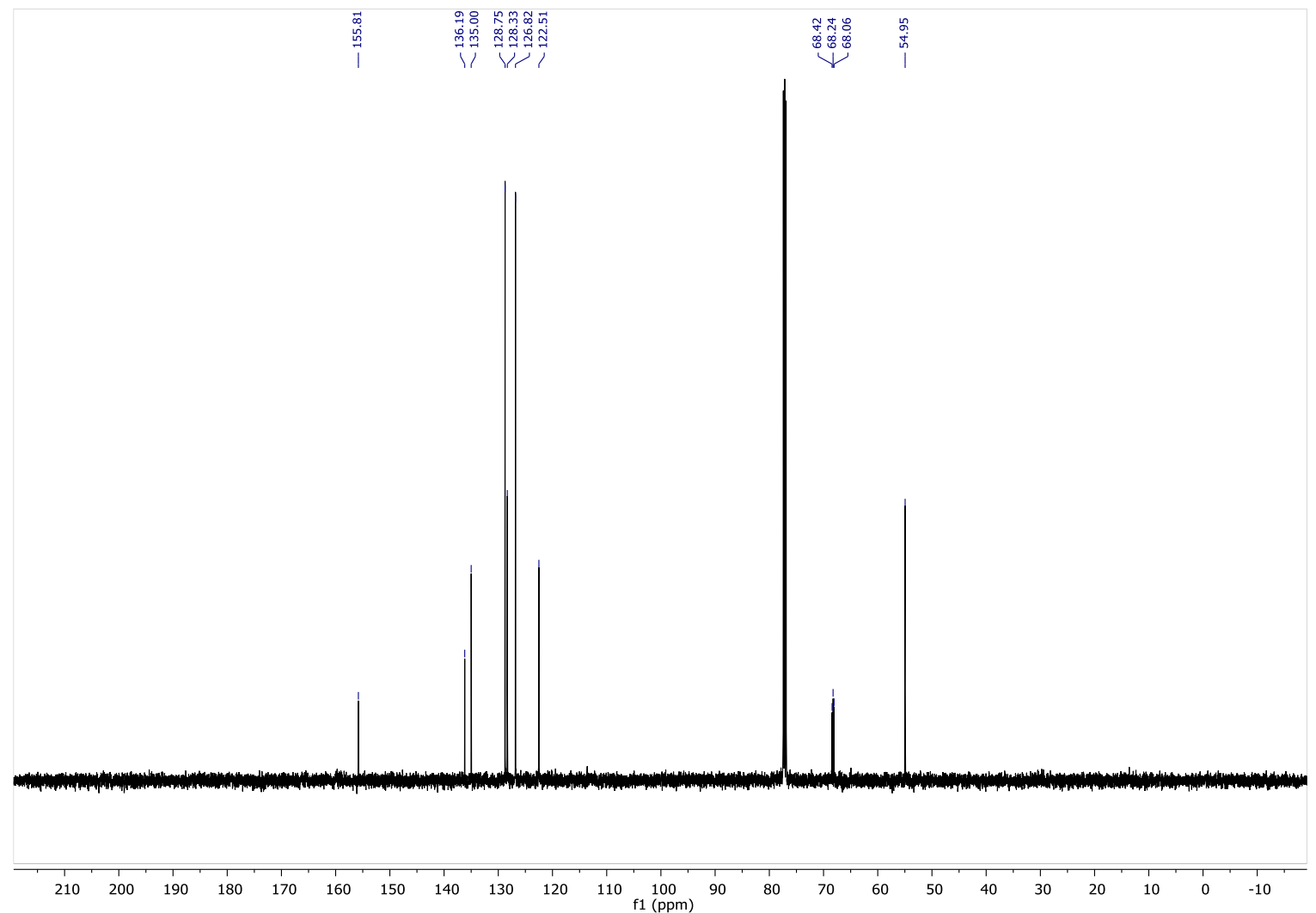

\section{References}

1. Lowry, M. S.; Goldsmith, J. I.; Slinker, J. D.; Rohl, R.; Pascal, R. A.; Malliaras, G. G.; Bernhard, S., SingleLayer Electroluminescent Devices and Photoinduced Hydrogen Production from an Ionic Iridium(III) Complex. Chem. Mater. 2005, 17 (23), 5712-5719.

2. Luo, J.; Zhang, J., Donor-Acceptor Fluorophores for Visible-Light-Promoted Organic Synthesis: Photoredox/Ni Dual Catalytic C(sp3)-C(sp2) Cross-Coupling. ACS Catal. 2016, 6 (2), 873-877.

3. Gutiérrez-Bonet, Á.; Tellis, J. C.; Matsui, J. K.; Vara, B. A.; Molander, G. A., 1,4-Dihydropyridines as Alkyl Radical Precursors: Introducing the Aldehyde Feedstock to Nickel/Photoredox Dual Catalysis. ACS Catal. 2016, 6 (12), 8004-8008.

4. Bai, X.-d.; Wang, J.; He, Y., Iridium-Catalyzed Propenylation Reactions for the Synthesis of 4-Pyridone Derivatives. Advanced Synthesis \& Catalysis 2019, 361 (3), 496-501.

5. Patel, N. R.; Kelly, C. B.; Jouffroy, M.; Molander, G. A., Engaging Alkenyl Halides with Alkylsilicates via Photoredox Dual Catalysis. Org. Lett. 2016, 18 (4), 764-767.

6. Ren, H.; Li, G.-F.; Zhu, B.; Lv, X.-D.; Yao, L.-S.; Wang, X.-L.; Su, Z.-M.; Guan, W., How Does Iridium(III) Photocatalyst Regulate Nickel(II) Catalyst in Metallaphotoredox-Catalyzed C-S Cross-Coupling? Theoretical and Experimental Insights. ACS Catal. 2019, 9 (5), 3858-3865.

7. Liu, J.; Wen, X.; Qin, C.; Li, X.; Luo, X.; Sun, A.; Zhu, B.; Song, S.; Jiao, N., Oxygenation of Simple Olefins through Selective Allylic $\mathrm{C}-\mathrm{C}$ Bond Cleavage: A Direct Approach to Cinnamyl Aldehydes. Angew. Chem., Int. Ed. 2017, 56 (39), 11940-11944.

8. Cao, H.; Jiang, H.; Feng, H.; Kwan, J. M. C.; Liu, X.; Wu, J., Photo-induced Decarboxylative Heck-Type Coupling of Unactivated Aliphatic Acids and Terminal Alkenes in the Absence of Sacrificial Hydrogen Acceptors. J. Am. Chem. Soc. 2018, 140 (47), 16360-16367. 